\begin{tabular}{|c|c|}
\hline Title & A continuum-mechanical formulation for shallow polythermal ice sheets \\
\hline Author(s) & Greve, Ralf \\
\hline Citation & $\begin{array}{l}\text { Philosophical Transactions of The Royal Society. A , Mathematical Physical and Engineering Sciences, 355(1726), 921- } \\
974 \\
\text { https://doi.org/10.1098/rsta.1997.0050 }\end{array}$ \\
\hline Issue Date & $1997-05-15$ \\
\hline Doc URL & http:/hdl.handle.net/2115/34856 \\
\hline Type & article (author version) \\
\hline File Information & Greve_1997_PhilT ransRSocLond.pdf \\
\hline
\end{tabular}

Instructions for use 


\title{
A continuum-mechanical formulation for shallow polythermal ice sheets
}

\author{
Ralf GReve \\ Institut für Mechanik \\ Technische Hochschule Darmstadt \\ Hochschulstr. 1, D-64289 Darmstadt
}

\begin{abstract}
This paper is concerned with a new theoretical approach to model grounded ice sheets in three dimensions. These are considered as polythermal, i.e., there will be regions with temperatures below the pressure melting point ("cold ice") and regions with temperatures exactly at the pressure melting point ("temperate ice"). In the latter, small quantities of water may occur.

Based on previous approaches, an improved theory of polythermal ice sheets is developed, which is founded on continuum-thermodynamic balance relations and jump conditions for mass, momentum and energy. The rheological behaviour is assumed to be that of an incompressible, nonlinear viscous and heat conducting fluid; because of the dependence of viscosity on temperature and on water content, the problem is thermo-mechanically coupled. After presenting analytic solutions for a simple geometry (ice sheet of uniform depth), the theory is subjected to a scaling procedure with the assumptions of a small aspect ratio (ratio between typical vertical dimension and typical horizontal dimension) and a small Froude number. This leads to the introduction of the polythermal shallow-ice approximation (SIA) equations.

Finally, as an application of the model to a real problem, a numerically computed steady-state solution for the Greenland Ice Sheet under present climate conditions is presented and compared with the real ice sheet.
\end{abstract}

\section{Introduction}

The Earth's cryosphere consists of several components. Ice sheets are extended ice masses with a base resting on solid land; they have formed by accumulated snowfall in the course of the millennia. Ice stored in the present ice sheets (essentially Antarctica and Greenland) represents by far the biggest part of today's ice volume on Earth; this paper is concerned with a new theoretical formulation of ice sheet motions. Alpine glaciers develop in the same way; however, they cover alpine regions of much less extent, typically valleys, and therefore their contribution to the cryosphere is small. Ice shelves are floating ice masses that are fed from the seaward mass flux of an ice sheet; they exist typically in large bays of an ice-covered continental shield. Sea ice is superficially frozen sea water, and soil ice is frozen water in the ground as it occurs in permafrost regions.

Ice appearing in glaciers and ice sheets exists in two fundamentally different states (when additional tracers of salt and sediment are neglected). Cold ice is characterized by a temperature below the pressure melting point and can be described as an incompressible, viscous and heat-conducting one-component fluid; the large ice sheets on Earth consist mainly of this type of ice. However, the temperature of temperate ice is exactly equal to the pressure melting point, so that it may contain small quantities of water in addition. Therefore, as opposed to cold ice, it must be regarded as a two-component fluid. In ice sheets, regions of temperate ice may exist in thin, near-basal layers, with significant consequences on the flow behaviour. Glaciers and ice sheets that are made up by cold as well as temperate regions are referred to as polythermal (see e.g. Paterson, 1994).

In the past, several models for the numerical simulation of ice sheets have been developed, becoming possible only due to the high calculation performance of modern computers. The first, a still vertically 
integrated model, is due to Mahaffy (1976), and has been applied to the Barnes Ice Cap in the Canadian Arctic. The first genuinely three-dimensional model due to Jenssen (1977) has been used to model the Greenland Ice Sheet, yet with a very low spatial resolution because of the limited computer capacities at that time. Numerous models of increasing sophistication followed, with applications to different problems such as the Greenland Ice Sheet, the Antarctic Ice Sheet, the glacial Laurentide Ice Sheet, the hypothetic glacial Tibetian Ice Sheet and so forth (Budd and Smith, 1982; Oerlemans, 1982; Herterich, 1988; Fastook and Chapman, 1989; Letréguilly et. al., 1991a,b; Abe-Ouchi, 1993; Calov, 1994; Huybrechts, 1994; Fabré et. al., 1995; Calov and Hutter, 1996). Especially remarkable are the simulations of the Antarctic Ice Sheet carried out with the Huybrechts model (e.g. Huybrechts and Oerlemans, 1988; Huybrechts, 1992; Huybrechts, 1993), in which the coupled ice sheet/ice shelf/lithosphere problem is modelled with high spatial resolution.

All these models neglect the possible influence of temperate ice regions. The temperature field is calculated in the entire ice sheet by solving the heat equation for cold ice, then temperatures that exceed the pressure melting point are retrospectively reset to the pressure melting point (this is subsequently referred to as "cold-ice method"). However, this is an oversimplified approach, because it does not account for the fact that the responses of cold and temperate ice regions are different, and those regions are two different phases, separated by a phase transition surface (the cold-temperate transition surface or "CTS") for which jump conditions for the physical quantities mass, momentum and energy must be fulfilled (Hutter, 1983; Müller, 1985). Moreover, the cold-ice method does not determine the water content in temperate ice that has a very pronounced influence on the ice viscosity (Lliboutry and Duval, 1985).

In this study we present a new continuum-mechanical formulation for polythermal ice sheets. It is in large parts similar to previous formulations (Fowler and Larson, 1978; Hutter, 1982; Blatter, 1991; Hutter, 1993); however, it contains some crucial new features, namely

- consideration of the contribution of the diffusive water flux to the total heat flux in temperate ice,

- a new formulation of the boundary conditions for a temperate ice base, where especially the different behaviour of temperate ice with and without water diffusion is incorporated,

- a new formulation of the transition conditions at the cold-temperate-transition surface (CTS), with the inclusion of water surface production at the CTS,

- three-dimensional derivation of the shallow-ice approximation (SIA) for polythermal ice.

Furthermore, we discuss two different types of solutions, namely (i) a semi-analytic solution for a simple geometry (ice sheet of uniform depth), and (ii) a numerically computed steady-state solution for the Greenland Ice Sheet under present climate conditions.

\section{The polythermal ice-sheet model}

A polythermal ice sheet consists of cold-ice regions as well as temperate-ice regions; in the latter, besides the ice, water may also be present. Below the ice sheet is the lithosphere, which is represented as a solid rock layer of approximately $100 \mathrm{~km}$ thickness that floats on the viscous asthenosphere. However, only the uppermost few kilometers of the lithosphere influence the thermal response of the ice sheet. The typical geometry is sketched in fig. 1 , where also a Cartesian coordinate system $(x, y, z)$ is introduced; $x$ and $y$ span the horizontal plane, $z$ is vertical and anti-parallel to the direction of the gravity acceleration.

The following field equations, boundary and transition conditions extend the previous formulations of Fowler and Larson (1978), Hutter (1982), Blatter (1991) and Hutter (1993).

\subsection{Field equations}

\subsubsection{Cold regions}

Cold ice is ice with a temperature below the pressure melting point. If additional tracers of salt, sediment, debris or air are neglected, it can be regarded as a viscous, heat-conducting, incompressible one-component 


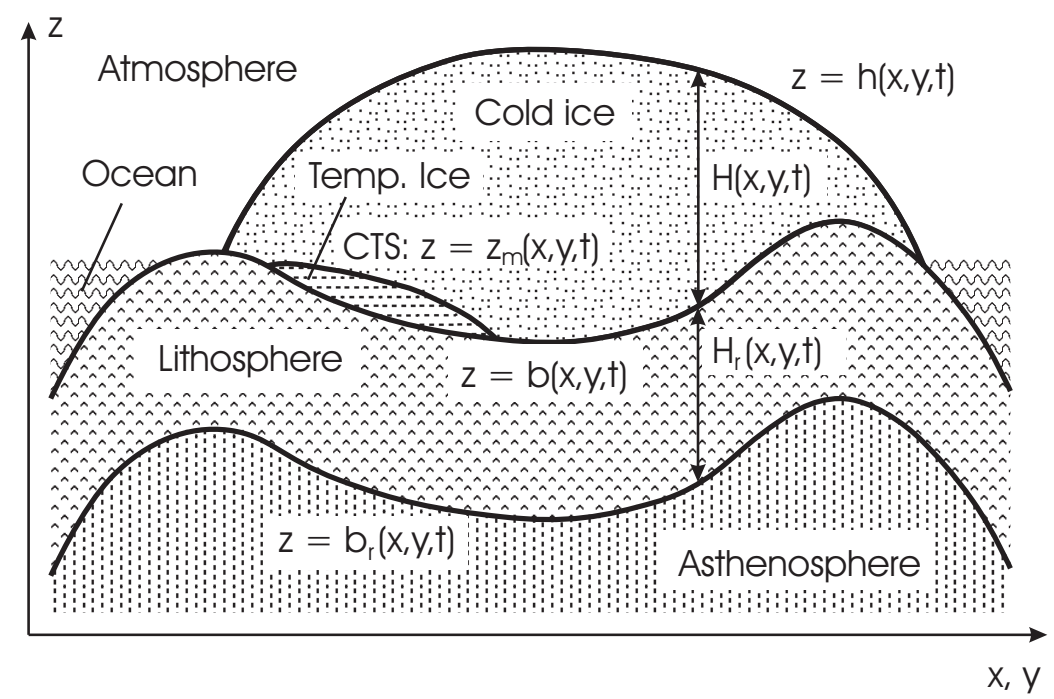

Figure 1: Sketch of a polythermal ice sheet, heavily exaggerated in the vertical. Definition of the Cartesian coordinate system used in this study: $x$ and $y$ span the horizontal plane, $z$ is the vertical coordinate.

fluid. Thus, the mass balance is given by

$$
\operatorname{div} \boldsymbol{v}=\operatorname{tr} \boldsymbol{D}=0,
$$

where $\boldsymbol{v}$ is the ice particle velocity, and $\boldsymbol{D}$ is the strain-rate tensor. Because of the incompressibility assumption, the stress tensor $\boldsymbol{T}$ must be split into an isotropic pressure tensor and a deviatoric (frictional) stress tensor,

$$
\boldsymbol{T}=-p \mathbf{1}+\boldsymbol{T}^{R}
$$

where the pressure $p$ is a free field, whereas the stress deviator $\boldsymbol{T}^{R}$ is described by a constitutive relation. The momentum balance is then

$$
-\operatorname{grad} p+\operatorname{div} \boldsymbol{T}^{R}+\rho \boldsymbol{g}=\rho \dot{\boldsymbol{v}}
$$

where $\boldsymbol{g}$ is the constant gravity acceleration, and a superposed dot denotes a material time derivative. The scaling analysis conducted below will show that the acceleration term $\rho \dot{\boldsymbol{v}}$ is negligible, so that pure Stokes flow prevails.

Three constitutive relations are required: a stress-strain-rate relation, a relation for the internal energy $\epsilon$ and one for the heat flux $\boldsymbol{q}$ (equal to the sensible heat flux $\boldsymbol{q}_{s}$ );

$$
\begin{gathered}
\boldsymbol{D}=E A\left(T^{\prime}\right) f(\sigma) \boldsymbol{T}^{R} \quad \text { with } \quad \sigma:=\sqrt{\frac{1}{2} \operatorname{tr}\left(\boldsymbol{T}^{R}\right)^{2},} \\
\dot{\epsilon}=c(T) \dot{T}, \\
\boldsymbol{q}=\boldsymbol{q}_{s}=-\kappa(T) \operatorname{grad} T,
\end{gathered}
$$

with in general temperature dependent specific heat $c$ and heat conductivity $\kappa$. The first equation implies that the ice fluidity factorizes into a function $A\left(T^{\prime}\right)$ ("rate factor") of the homologous temperature $T^{\prime}$ and a function $f(\sigma)$ ("creep response function") of the effective shear stress $\sigma$ (square root of the second invariant of the stress deviator $I I_{T^{R}}=\frac{1}{2} \operatorname{tr}\left(\boldsymbol{T}^{R}\right)^{2}$, cf. Hutter (1983)); the homologous temperature is defined as $T^{\prime}=T-T_{M}$, where $T_{M}$ is the pressure melting point of ice. The rate factor and the creep response function are not specified at this stage; the additional factor $E$ ("enhancement factor") can be set greater than unity to account, for instance, for the increased softness of glacial dust-containing ice compared with ordinary interglacial ice (Paterson, 1994). The second equation relates internal energy changes to that of temperature, and the last equation is Fourier's law for heat conduction. 
Neglecting heat supply due to radiation, the energy balance takes the form

$$
\rho \dot{\epsilon}=-\operatorname{div} \boldsymbol{q}+\operatorname{tr}\left(\boldsymbol{T}^{R} \boldsymbol{D}\right) .
$$

Introduction of the three constitutive relations (2.4) - (2.6) transforms this into an evolution equation for the temperature field,

$$
\rho c \dot{T}=\operatorname{div}(\kappa \operatorname{grad} T)+2 E A\left(T^{\prime}\right) f(\sigma) \sigma^{2} .
$$

This equation balances local temperature changes with advection (implicitly included in the material time derivative), heat conduction and dissipative strain heating.

\subsubsection{Temperate regions}

As opposed to cold ice, the temperature of temperate ice is exactly at the pressure melting point, so that it need not be calculated separately, but follows immediately from the pressure field:

$$
T=T_{M}=T_{0}-\beta^{*} p=T_{0}-\beta \frac{p}{\rho g},
$$

where $T_{0}=0^{\circ} \mathrm{C}, \beta^{*}$ is the Clausius-Clapeyron constant (Paterson, 1994), and the Clausius-Clapeyron gradient $\beta:=\rho g \beta^{*}$ corresponds to the temperature gradient in temperate ice as shown below. Temperate ice may contain a certain amount of water; as the main thermodynamic quantity the water content (more precisely: mass fraction $\omega$ ) takes the role of temperature in cold ice. Therefore, in contrast to cold ice, temperate ice must be regarded as a binary mixture of ice and water, and $\rho$ denotes the total mixture density. Because of this, it is necessary to apply some basic concepts of mixture theory (cf. Müller, 1985). Owing to the general assumption that the water content in temperate zones of polythermal ice sheets is small, with maximum values of about $5 \%$ (Hutter, 1993), temperate ice will be described by two mass balances (one for the mixture as a whole, one for the component water), but only one momentum and one energy balance for the mixture. That is, water is considered as a tracer component whose motion relative to the barycentre of the mixture is described by Fickian diffusion. Alternative concepts not necessary in the present study, but more appropriate for polythermal alpine glaciers with sometimes very high water content, include two separate momentum balances with a Darcy-type interaction force between the two components (Fowler, 1984; Hutter, 1993; Morland, 1993).

Before the field equations for temperate ice can be formulated, some quantities from mixture theory must be introduced. The barycentric velocity is defined as

$$
\boldsymbol{v}:=\frac{1}{\rho}\left(\rho_{i} \boldsymbol{v}_{i}+\rho_{w} \boldsymbol{v}_{w}\right)
$$

The indices $i$ and $w$, respectively, refer to the components ice and water; $\rho_{i / w}$ then denote the corresponding partial densities. The water content is introduced as the mass fraction, $\omega$, of water in the mixture, namely,

$$
\omega:=\frac{\rho_{w}}{\rho} .
$$

In addition, a diffusive water mass flux $\boldsymbol{j}$ is defined, that describes the water motion relative to the motion of the barycentre,

$$
\boldsymbol{j}:=\rho_{w}\left(\boldsymbol{v}_{w}-\boldsymbol{v}\right)=\rho \omega\left(\boldsymbol{v}_{w}-\boldsymbol{v}\right) .
$$

As was the case for cold ice, the mixture is also assumed incompressible, i.e., $\rho$ is constant. This is problematic in so far as the intrinsic densities of ice and water are distinctly different (according to Paterson (1994) the density of glacier ice varies in the range of $830-910 \mathrm{~kg} / \mathrm{m}^{3}$, whereas the density of water is $1000 \mathrm{~kg} / \mathrm{m}^{3}$ ). However, because of the assumption of approximately $5 \%$ maximum water content, the relative changes of mixture density due to changes of the water content do not exceed $1 \%$ and are therefore negligible. As a consequence, the mixture mass balance and the mixture momentum balance have the same form as for cold ice, namely

$$
\begin{gathered}
\operatorname{div} \boldsymbol{v}=0, \\
-\operatorname{grad} p+\operatorname{div} \boldsymbol{T}^{R}+\rho \boldsymbol{g}=\rho \dot{\boldsymbol{v}},
\end{gathered}
$$


where the stress tensor $\boldsymbol{T}$ has again been decomposed as $\boldsymbol{T}=-p \mathbf{1}+\boldsymbol{T}^{R}$.

When formulating the mass balance for the component water, it must be noted that the partial density of water $\rho_{w}$ is not constant, but depends on the water content itself. Furthermore, the mass of water is not conserved due to the possibility of melting and freezing processes. It is therefore necessary to include a production term $M$, the rate of water mass produced per unit mixture volume, then

$$
\frac{\partial \rho_{w}}{\partial t}+\operatorname{div}\left(\rho_{w} \boldsymbol{v}_{w}\right)=M,
$$

which is equivalent to

$$
\rho \dot{\omega}=-\operatorname{div} \boldsymbol{j}+M .
$$

As was the case for cold ice, constitutive relations are required to close the system. These are (see Hutter, 1993);

$$
\begin{gathered}
\boldsymbol{D}=E A_{t}(\omega) f_{t}(\sigma) \boldsymbol{T}^{R}, \\
\dot{\epsilon}=L \dot{\omega}+c(T) \dot{T}_{M}, \\
\boldsymbol{j}=-\nu \operatorname{grad} \omega, \\
\boldsymbol{q}_{s}=-\kappa(T) \operatorname{grad} T_{M} .
\end{gathered}
$$

The first equation, namely the stress-strain-rate relation, is the counterpart of (2.4) for cold ice; however, the temperature dependence of the rate factor is replaced by a factor depending on the water content (function $A_{t}(\omega)$ ). The second equation relates changes of the internal energy to changes of the water content and of the melting temperature ${ }^{1}$. The third equation is the Fickian diffusion law already mentioned above, and the last equation is again Fourier's law for heat conduction; however, here the total heat flux is not equal to the sensible heat flux (see below). The latent heat $L$ and water diffusivity $\nu$ are assumed to have constant values.

Next, consider the mixture energy balance. In (2.18) the internal energy $\epsilon$ depends on the water content $\omega$, so that a non-vanishing diffusive water flux $\boldsymbol{j}$ contributes to a flux of internal energy (latent heat flux $\left.\boldsymbol{q}_{l}=L \boldsymbol{j}\right)$. Therefore, the total heat flux $\boldsymbol{q}$ can be expressed as

$$
\boldsymbol{q}=\boldsymbol{q}_{s}+\boldsymbol{q}_{l}=\boldsymbol{q}_{s}+L \boldsymbol{j} .
$$

The inclusion of the additional term $L \boldsymbol{j}$ is a new feature of the theoretical formulation presented here. With this modified form of the energy flux, the mixture energy balance becomes

$$
\rho \dot{\epsilon}=-\operatorname{div}\left(\boldsymbol{q}_{s}+L \boldsymbol{j}\right)+\operatorname{tr}\left(\boldsymbol{T}^{\boldsymbol{R}} \boldsymbol{D}\right) .
$$

Introducing the constitutive relations $(2.17)-(2.20)$ into the water mass balance (2.16) and into the mixture energy balance (2.22) yields the respective relations

$$
\rho \dot{\omega}=\nu \nabla^{2} \omega+M,
$$

and

$$
\rho L \dot{\omega}+\rho c \dot{T}_{M}=L \nu \nabla^{2} \omega+\operatorname{div}\left(\kappa \operatorname{grad} T_{M}\right)+2 E A_{t}(\omega) f_{t}(\sigma) \sigma^{2},
$$

which are consistent, provided that the water production rate $M$ is given by

$$
M=\frac{1}{L}\left(2 E A_{t}(\omega) f_{t}(\sigma) \sigma^{2}+\operatorname{div}\left(\kappa \operatorname{grad} T_{M}\right)-\rho c \dot{T}_{M}\right) .
$$

This has the physical interpretation that the energy available for melting is composed of three terms: (i) the heat dissipated by stress power, $\operatorname{tr}\left(\boldsymbol{T}^{R} \boldsymbol{D}\right)=2 E A_{t}(\omega) f_{t}(\sigma) \sigma^{2}$, (ii) the heat conducted to the point under consideration and (iii) the heat stored by changes in the melting temperature (the latter term being obviously negative for $\dot{T}_{M}>0$ ). Of course, the latter two effects contribute little to the value of $L M$, but this is exactly what is expected in an environment with two coexisting, exchanging phases. In earlier theories in which the latent heat flux $L \boldsymbol{j}$ was not included in $\boldsymbol{q}$, there is an additional contribution

\footnotetext{
${ }^{1}$ From a strict thermodynamical point of view this relation is merely approximate (Svendsen, pers. comm.).
} 
$-\nu \nabla^{2} \omega$ to $M$, a heat source due to the water flux. This ambiguity arises because separate momentum and energy balances for water and ice are not considered. Strictly mixture-theoretical computation of $\boldsymbol{q}$ shows, however, that the latent heat flux $L \boldsymbol{j}$, which arises from water diffusion, contributes indeed to the mixture heat flux $\boldsymbol{q}$, as is typical for fluxes in mixtures (Müller, 1985; Svendsen, pers. comm.), so that our approach seems to be preferable. Moreover, if the latent heat flux were ignored, the additional term $-\nu \nabla^{2} \omega$ in $M$ would cancel water diffusion in the water mass balance (2.23) and therefore in the final water-content equation (4.137), although diffusion was originally included in the model, a physically very questionable result.

\subsubsection{Lithosphere}

Since the objective of this work is ice sheet modelling, in the solid rock (lithosphere) only those processes are included that are of some relevance for the ice sheet. These are (i) the heat conduction in the lithosphere and the resulting thermal inertia effect on the ice sheet, and (ii) the isostatic adjustment as a consequence of the varying ice load.

Analogous to the procedure for cold ice, the temperature equation in the rock becomes (see (2.8))

$$
\rho_{r} c_{r} \dot{T}=\kappa_{r} \nabla^{2} T .
$$

The index $(\cdot)_{r}$ refers to the lithosphere (= rock bed), so $\rho_{r}, c_{r}$ and $\kappa_{r}$ are its density, specific heat and heat conductivity, respectively. In contrast to cold ice, $c_{r}$ and $\kappa_{r}$ are assumed constant, and strain heating is neglected.

For the sinking depth $\Delta b(x, y, t)$ of the lithosphere into the asthenosphere below it, consider a local force balance between buoyancy and ice load for a vertical column of transect area $d A$ with ice thickness $H=h-b$ :

$$
\rho_{a} g \Delta b d A=\rho g H d A,
$$

where $\rho_{a}$ is the density of the asthenosphere. In this affirmation vertically moving lithosphere columns do not interact with each other, and have no horizontal velocity. With the relaxed ice-free steady-state lithosphere position at $z=b_{0}(x, y, t)$, its general steady-state position, $b_{\mathrm{ss}}$, is given by

$$
b_{\mathrm{ss}}=b_{0}-\Delta b=b_{0}-\frac{\rho}{\rho_{a}} H .
$$

Due to the asthenosphere viscosity, this equilibrium is not reached instantaneously, but with a certain time $\operatorname{lag} \tau_{V}$. The evolution equation for the position of the lithosphere surface at $z=b(x, y, t)$ is (Herterich, 1990)

$$
\frac{d b}{d t} \equiv \frac{\partial b}{\partial t}=-\frac{1}{\tau_{V}}\left(b-b_{\mathrm{ss}}\right)=-\frac{1}{\tau_{V}}\left[b-\left(b_{0}-\frac{\rho}{\rho_{a}} H\right)\right] .
$$

For a fixed ice thickness $H$ this corresponds to an exponential approach of $b$ towards the equilibrium state $b_{\mathrm{ss}}$.

Under the additional assumption that each vertical column of the lithosphere is rigid, the velocity field in the lithosphere is

$$
\boldsymbol{v}=\frac{\partial b}{\partial t}(x, y, t) \boldsymbol{e}_{z},
$$

where $\boldsymbol{e}_{z}$ is the unit vector pointing in the $z$ direction.

\subsection{Boundary and transition conditions}

\subsubsection{Boundary conditions at the free surface}

As for any singular surface, at the free surface of the ice sheet (ice-atmosphere interface) a kinematic condition can be formulated. If the free surface is given implicitly by the equation $F_{s}(\boldsymbol{x}, t)=0$ (fig. 2), with positive side adjacent to the atmosphere, the normal unit vector $\boldsymbol{n}=\operatorname{grad} F_{s} /\left\|\operatorname{grad} F_{s}\right\|$ points into the atmosphere. Therefore, the time derivative of $F_{s}$ following the motion of the free surface with velocity 
$\boldsymbol{w}$ must vanish,

$$
\frac{d^{w} F_{s}}{d t}=\frac{\partial F_{s}}{\partial t}+\boldsymbol{w} \cdot \operatorname{grad} F_{s}=0 .
$$

Then, introducing the ice volume flux through the free surface, $a_{s}^{\perp}:=\left(\boldsymbol{w}-\boldsymbol{v}^{-}\right) \cdot \boldsymbol{n}$,

$$
\frac{\partial F_{s}}{\partial t}+\boldsymbol{v}^{-} \cdot \operatorname{grad} F_{s}=-\left\|\operatorname{grad} F_{s}\right\| \cdot a_{s}^{\perp} .
$$

With the Cartesian representation of the free surface $z=h(x, y, t), F_{s}(\boldsymbol{x}, t):=z-h(x, y, t)$, and thus

$$
\frac{\partial h}{\partial t}+v_{x}^{-} \frac{\partial h}{\partial x}+v_{y}^{-} \frac{\partial h}{\partial y}-v_{z}^{-}=\left(1+\left(\frac{\partial h}{\partial x}\right)^{2}+\left(\frac{\partial h}{\partial y}\right)^{2}\right)^{1 / 2} a_{s}^{\perp}
$$

The ice volume flux through the free surface, $a_{s}^{\perp}$ ("accumulation-ablation function"), is a climatic input quantity, composed of surface snowfall rate $S_{s}$ (accumulation) minus surface melting rate $M_{s}$ (ablation).

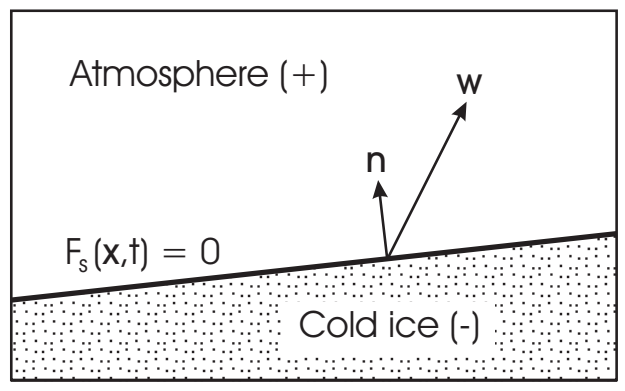

Figure 2: Geometry of the free surface.

From the general momentum jump relation (Müller, 1985) there follows, apart from the extremely small convective momentum flux through the free surface, the continuity of the traction $\boldsymbol{T n}$. Neglecting the stresses on the atmosphere side, composed of the atmospheric pressure $p_{\text {atm }}$ and the wind shear stress $\boldsymbol{\tau}_{\text {wind }}$, which are small compared to the stresses in the ice sheet,

$$
\boldsymbol{T}^{-} \boldsymbol{n}=\boldsymbol{T}^{+} \boldsymbol{n}=-p_{\text {atm }} \boldsymbol{n}+\boldsymbol{\tau}_{\text {wind }}=\mathbf{0}
$$

Further, in the case of a cold free surface (the usual situation) the surface temperature will be prescribed,

$$
T^{-}(\boldsymbol{x}, t)=T_{s}(\boldsymbol{x}, t)
$$

so, $T_{s}$ represents a further climatic input quantity. In reality, the firn temperature at 10 meters depth, where seasonal temperature variations (that are irrelevant for the time scales on which ice sheet dynamics takes place) are damped to a maximum of $1 \%$ of their surface amplitudes, is interpreted as $T_{s}$. At a temperate free surface patch, which can possibly arise in small regions close to the ice-sheet margin, the water content $\omega$ or, alternatively, its normal derivative must be prescribed instead.

\subsubsection{Transition conditions at the cold ice base}

Because of the different properties of cold and temperate ice, the transition conditions between ice and bedrock (lithosphere) must distinguish the two cases of (i) an ice base below pressure melting (cold ice base) and (ii) an ice base at pressure melting (temperate ice base). Consider first the case of a cold ice base.

The bedrock below the ice sheet will be assumed to be impermeable, i.e., a possible mass exchange between cold ice and lithosphere is ignored. The ice sheet base is denoted by $z=b(x, y, t)($ fig. 3$)$; the positive side is identified with the lithosphere, the negative side with the ice. By setting $F_{b}(\boldsymbol{x}, t):=$ $b(x, y, t)-z$, the normal unit vector $\boldsymbol{n}=\operatorname{grad} F_{b} /\left\|\operatorname{grad} F_{b}\right\|$ then points into the lithosphere. These definitions hold for both a cold and a temperate ice base. 


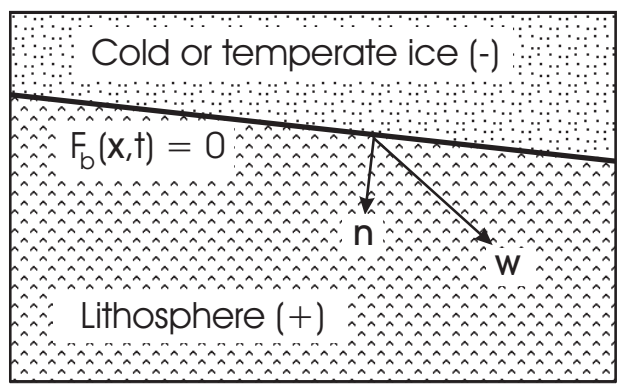

Figure 3: Geometry of the ice base.

Because of the assumed impermeability,

$$
\left(\boldsymbol{v}^{+}-\boldsymbol{w}\right) \cdot \boldsymbol{n}=\left(\boldsymbol{v}^{-}-\boldsymbol{w}\right) \cdot \boldsymbol{n}=0
$$

so that the general mass jump relation (Müller, 1985) is identically satisfied. The kinematic condition (see eq. (2.32) for the free surface) is therefore

$$
\frac{\partial F_{b}}{\partial t}+\boldsymbol{v}^{-} \cdot \operatorname{grad} F_{b}=0
$$

or

$$
\frac{\partial b}{\partial t}+v_{x}^{-} \frac{\partial b}{\partial x}+v_{y}^{-} \frac{\partial b}{\partial y}-v_{z}^{-}=0 .
$$

Further, a sliding law that relates the basal sliding velocity $\boldsymbol{v}_{\mathrm{sl}}:=\boldsymbol{v}_{\|}^{-}-\boldsymbol{v}_{\|}^{+}\left(\right.$where $\left.\boldsymbol{v}_{\|}^{ \pm}=\boldsymbol{v}^{ \pm}-\left(\boldsymbol{v}^{ \pm} \cdot \boldsymbol{n}\right) \boldsymbol{n}\right)$ to the basal shear stress $\boldsymbol{t}_{\|}^{-}$is introduced:

$$
\boldsymbol{v}_{\mathrm{sl}}=-C\left(t_{\perp}^{-}, \ldots\right) \boldsymbol{t}_{\|}^{-}
$$

with $t_{\perp}^{-}=\boldsymbol{n} \cdot \boldsymbol{T}^{-} \boldsymbol{n}$ and $\boldsymbol{t}_{\|}^{-}=\boldsymbol{T}^{-} \boldsymbol{n}-t_{\perp}^{-} \boldsymbol{n}$. The sliding function $C$ depends on the normal basal traction $t_{\perp}^{-}$ and possibly on further scalar quantities such as $\left\|\boldsymbol{t}_{\|}^{-}\right\|$or $T$. Usually in the case of a cold ice base $C=0$, i.e., adhesion of the basal ice at the bedrock, is assumed in ice-sheet and glacier models.

With the impermeability relation (2.36), the general momentum jump relation implies

$$
\llbracket \boldsymbol{T n} \rrbracket=0,
$$

i.e., continuity of the stress vector.

In addition, the energy jump relation is required. With (2.36) and (2.40), the general form (Müller, 1985) reduces to

$$
\kappa\left(\operatorname{grad} T^{-} \cdot \boldsymbol{n}\right)-\kappa_{r}\left(\operatorname{grad} T^{+} \cdot \boldsymbol{n}\right)=\llbracket \boldsymbol{v} \rrbracket \cdot \boldsymbol{T}^{-} \boldsymbol{n}=-\boldsymbol{v}_{\mathrm{sl}} \cdot \boldsymbol{T}^{-} \boldsymbol{n} .
$$

The term on the right-hand side represents the basal frictional heating, which vanishes in the case of basal adhesion. Finally, the temperature is assumed continuous,

$$
\llbracket T \rrbracket=0 .
$$

\subsubsection{Transition conditions at the temperate ice base}

Next consider a temperate ice base, where the temperature at the ice-lithosphere interface is at pressure melting. However, the presence of a temperate ice base at some place in the ice sheet does not necessarily entail the occurence of a temperate ice layer of non-vanishing thickness above it. It is equally possible that the temperature gradient at the ice base is below the Clausius-Clapeyron gradient, so that the ice becomes cold immediately above the base, even though the base itself is temperate. The relations derived in this section hold for the case of a basal temperate ice layer as well as for the case of a pure temperate 
ice base overlain by cold ice.

The description of the geometry of the cold ice base also applies here. However, the impermeability assumption applied at the cold ice base does not follow since the possibility of water drainage into the bedrock is not excluded.

The mass jump relation for the component water is

$$
\llbracket \rho_{w}\left(\boldsymbol{v}_{w}-\boldsymbol{w}\right) \cdot \boldsymbol{n} \rrbracket=\mathcal{P}_{b}^{w},
$$

where a surface production rate of water $\mathcal{P}_{b}^{w}$ has been introduced in order to describe the basal ice melting due to the geothermal heat flux and the basal frictional heating caused by sliding. This can be transformed to

$$
\rho \omega^{-}\left(\boldsymbol{v}_{w}^{-}-\boldsymbol{w}\right) \cdot \boldsymbol{n}=\dot{m}_{b}^{w}-\mathcal{P}_{b}^{w}
$$

here the water mass flux into the base, $\dot{m}_{b}^{w}:=\rho_{w}^{+}\left(\boldsymbol{v}_{w}^{+}-\boldsymbol{w}\right) \cdot \boldsymbol{n}$, has been defined, representing a boundary condition that must be prescribed in general.

Analogous, the mass jump relation for the component ice yields

$$
\llbracket \rho_{i}\left(\boldsymbol{v}_{i}-\boldsymbol{w}\right) \cdot \boldsymbol{n} \rrbracket=-\mathcal{P}_{b}^{w} .
$$

Since the lithosphere is impermeable to ice, and therefore does not contain any ice $\left(\rho_{i}^{+}=0\right)$,

$$
\rho\left(1-\omega^{-}\right)\left(\boldsymbol{v}_{i}^{-}-\boldsymbol{w}\right) \cdot \boldsymbol{n}=\mathcal{P}_{b}^{w} .
$$

With the definition (2.10) of the barycentric velocity, it follows that $\boldsymbol{v}-\boldsymbol{w}=\omega\left(\boldsymbol{v}_{w}-\boldsymbol{w}\right)+(1-\omega)\left(\boldsymbol{v}_{i}-\boldsymbol{w}\right)$, which upon scalar multiplication by $\boldsymbol{n}$ and use of (2.44) and (2.46) becomes

$$
\left(\boldsymbol{v}^{-}-\boldsymbol{w}\right) \cdot \boldsymbol{n}=\frac{\dot{m}_{b}^{w}}{\rho} .
$$

In the case of a negligible diffusive water flux $\boldsymbol{j}\left(\right.$ i.e., $\left.\boldsymbol{v}_{w}=\boldsymbol{v}_{i}=\boldsymbol{v}\right)$, the water mass flux into the base $\dot{m}_{b}^{w}$ can be calculated by comparing (2.46) and (2.47),

$$
\dot{m}_{b}^{w}=\frac{\mathcal{P}_{b}^{w}}{1-\omega^{-}},
$$

and therefore, in contrast to the general case, does not need separate prescription.

By applying (2.47), the kinematic condition (compare with eqs. (2.32) and (2.33) for the free surface) becomes

$$
\frac{\partial F_{b}}{\partial t}+v^{-} \cdot \operatorname{grad} F_{b}=\left\|\operatorname{grad} F_{b}\right\| \cdot \frac{\dot{m}_{b}^{w}}{\rho},
$$

or

$$
\frac{\partial b}{\partial t}+v_{x}^{-} \frac{\partial b}{\partial x}+v_{y}^{-} \frac{\partial b}{\partial y}-v_{z}^{-}=\left(1+\left(\frac{\partial b}{\partial x}\right)^{2}+\left(\frac{\partial b}{\partial y}\right)^{2}\right)^{1 / 2} \frac{\dot{m}_{b}^{w}}{\rho} .
$$

For the diffusive water flux $\boldsymbol{j}$, from (2.44) and (2.47),

$$
\begin{aligned}
\boldsymbol{j}^{-} \cdot \boldsymbol{n} & =\rho \omega^{-}\left(\boldsymbol{v}_{w}^{-}-\boldsymbol{w}\right) \cdot \boldsymbol{n}-\omega^{-}\left\{\rho\left(\boldsymbol{v}^{-}-\boldsymbol{w}\right) \cdot \boldsymbol{n}\right\} \\
& =\dot{m}_{b}^{w}-\mathcal{P}_{b}^{w}-\omega^{-} \dot{m}_{b}^{w} \\
& =\left(1-\omega^{-}\right) \dot{m}_{b}^{w}-\mathcal{P}_{b}^{w} .
\end{aligned}
$$

Together with the diffusion law (2.19), (2.51) represents a mixed boundary condition for the basal water content.

As for the case of a cold ice base, a sliding law

$$
\boldsymbol{v}_{\mathrm{sl}}=-C_{t}\left(t_{\perp}^{-}, \ldots\right) \boldsymbol{t}_{\|}^{-}
$$

is formulated, with $\boldsymbol{v}_{\mathrm{sl}}:=\boldsymbol{v}_{\|}^{-}-\boldsymbol{v}_{\|}^{+}, t_{\perp}^{-}=\boldsymbol{n} \cdot \boldsymbol{T}^{-} \boldsymbol{n}$ and $\boldsymbol{t}_{\|}^{-}=\boldsymbol{T}^{-} \boldsymbol{n}-t_{\perp}^{-} \boldsymbol{n}$ as above. Since the water in 
temperate ice may act as a lubricating film between ice and rock, the sliding function $C_{t}$ is expected to be distinctly larger than its cold-ice counterpart $C$.

Neglecting the small convective momentum flux resulting from (2.47), the momentum jump relation yields again the continuity of the traction,

$$
\llbracket \boldsymbol{T n} \rrbracket=0 .
$$

Ignoring the contribution from the kinetic energy, the energy jump relation for the mixture takes the form

$$
\llbracket \boldsymbol{q}_{s} \cdot \boldsymbol{n} \rrbracket+L \llbracket \boldsymbol{j} \cdot \boldsymbol{n} \rrbracket-\llbracket \boldsymbol{v} \cdot \boldsymbol{T} \boldsymbol{n} \rrbracket+\llbracket(\rho(\boldsymbol{v}-\boldsymbol{w}) \cdot \boldsymbol{n}) L \omega \rrbracket=0,
$$

and with the relation $(2.51)$ this can be simplified to

$$
\kappa\left(\operatorname{grad} T^{-} \cdot \boldsymbol{n}\right)-\kappa_{r}\left(\operatorname{grad} T^{+} \cdot \boldsymbol{n}\right)+\boldsymbol{v}_{\mathrm{s} l} \cdot \boldsymbol{T}^{-} \boldsymbol{n}+\llbracket \rho L \omega\left(\boldsymbol{v}_{w}-\boldsymbol{w}\right) \cdot \boldsymbol{n} \rrbracket=0,
$$

or, in view of (2.43),

$$
\mathcal{P}_{b}^{w}=\frac{1}{L}\left(\kappa_{r}\left(\operatorname{grad} T^{+} \cdot \boldsymbol{n}\right)-\kappa\left(\operatorname{grad} T^{-} \cdot \boldsymbol{n}\right)-\boldsymbol{v}_{\mathrm{sl}} \cdot \boldsymbol{T}^{-} \boldsymbol{n}\right)
$$

This result is clear; it says that the water production rate at the temperate base, $\mathcal{P}_{b}^{w}$ ("basal melting rate"), is fed by the heat fluxes flowing toward the interface from the ice and from the lithosphere as well as by the basal heat production due to sliding. The temperature is continuous,

$$
\llbracket T \rrbracket=0 \quad \Rightarrow \quad T^{+}=T^{-}=T_{M},
$$

with the phase change surface at the melting temperature. In contrast to the cold ice base, the temperature $T_{M}$ at the temperate ice base is known (provided the pressure field is known), achieving a thermal decoupling between the ice domain and the lithosphere domain underneath.

\subsubsection{Boundary conditions at the lithosphere base}

The base of the modelled lithosphere is situated at $z=b_{r}(x, y, t)$, or implicitly $F_{r}(\boldsymbol{x}, t):=b_{r}(x, y, t)-z=0$ as above. The positive side is identified with the asthenosphere and the negative side with the lithosphere, and the normal unit vector $\boldsymbol{n}=\operatorname{grad} F_{r} /\left\|\operatorname{grad} F_{r}\right\|$ therefore points into the asthenosphere (fig. 4).

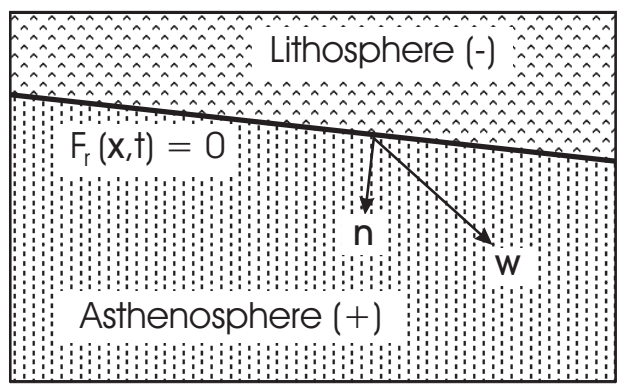

Figure 4: Geometry of the lithosphere base.

Within the framework of the simple lithosphere model applied here, it is merely required to prescribe a thermal boundary condition at the lithosphere base. To achieve this, specify the geothermal heat flux $Q_{\text {geoth }}^{\perp}:=-\boldsymbol{q}^{-} \cdot \boldsymbol{n}$ in the lithosphere, then with a Fourier heat conduction law

$$
\kappa_{r}\left(\operatorname{grad} T^{-} \cdot \boldsymbol{n}\right)=Q_{\text {geoth }}^{\perp},
$$

which is a Neumann condition for the temperature. 


\subsubsection{Transition conditions at the CTS}

The CTS ("cold-temperate transition surface") constitutes the phase-change surface between the cold and temperate regions of an ice sheet and is therefore, like the free surface and the ice base, a singular surface at which the physical quantities may suffer jumps. Its geometry is sketched in fig. 5 . It will be described explicitly by $z=z_{m}(x, y, t)$, and thus implicitly by $F_{m}(\boldsymbol{x}, t):=z-z_{m}(x, y, t)=0$; the positive side is the upper (cold ice) side, the negative side the lower (temperate ice) side, so that the normal unit vector $\boldsymbol{n}=\operatorname{grad} F_{m} /\left\|\operatorname{grad} F_{m}\right\|$ points into the cold ice.

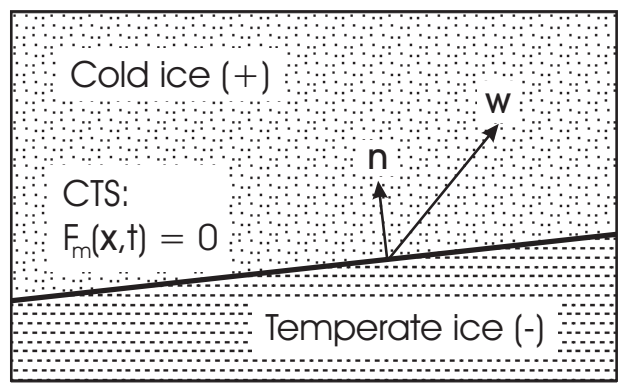

Figure 5: Geometry of the CTS.

First, the kinematic condition,

$$
\frac{\partial F_{m}}{\partial t}+\boldsymbol{v} \cdot \operatorname{grad} F_{m}=-\left\|\operatorname{grad} F_{m}\right\| \cdot a_{m}^{\perp},
$$

with the above choice for $F_{m}$, becomes

$$
\frac{\partial z_{m}}{\partial t}+v_{x} \frac{\partial z_{m}}{\partial x}+v_{y} \frac{\partial z_{m}}{\partial y}-v_{z}=\left(1+\left(\frac{\partial z_{m}}{\partial x}\right)^{2}+\left(\frac{\partial z_{m}}{\partial y}\right)^{2}\right)^{1 / 2} a_{m}^{\perp}
$$

In this equation the ice volume flux through the CTS, $a_{m}^{\perp}:=(\boldsymbol{w}-\boldsymbol{v}) \cdot \boldsymbol{n}$, has been introduced. This sign choice causes $a_{m}^{\perp}$ to be positive for melting conditions (ice flow direction from the cold into the temperate region) and negative for freezing conditions (ice flow direction from the temperate into the cold region). Because of the continuity of $\boldsymbol{v}$ across the CTS to be deduced below it is not necessary to distinguish between $\boldsymbol{v}^{+}$und $\boldsymbol{v}^{-}$. In contrast to the accumulation-ablation function $a_{s}^{\perp}$ on the free surface, $a_{m}^{\perp}$ arises in the interior of the ice sheet, and in consequence must be computed by the model.

The temperature and the tangential velocity are assumed continuous,

$$
\llbracket T \rrbracket=0, \quad \llbracket \boldsymbol{v}-(\boldsymbol{v} \cdot \boldsymbol{n}) \boldsymbol{n} \rrbracket=\mathbf{0} .
$$

When deriving the mass balance for temperate ice it was noted that the density difference between cold and temperate ice is at most $1 \%$. If this slight difference is ignored, the mass jump relation yields continuity for the normal velocity at the CTS as well,

$$
\llbracket \boldsymbol{v} \cdot \boldsymbol{n} \rrbracket=0,
$$

so that the entire velocity vector is continuous,

$$
\llbracket v \rrbracket=0 .
$$

From this and from the momentum jump relation follows the continuity of traction,

$$
\llbracket \boldsymbol{T n} \rrbracket=0 .
$$

Now consider the mass jump relation for the component water. Since melting and freezing processes may 
occur at the CTS, a surface production term $\mathcal{P}_{m}^{w}$ for the component water must be introduced. Then

$$
\llbracket \rho_{w}\left(\boldsymbol{v}_{\boldsymbol{w}}-\boldsymbol{w}\right) \cdot \boldsymbol{n} \rrbracket=\mathcal{P}_{m}^{w},
$$

or, equivalently, with the diffusive water flux given by (2.12) (in view of the fact that at the positive (cold) side of the CTS no water is present, so that the quantities $\omega^{+}$and $\boldsymbol{j}^{+}$vanish)

$$
-\boldsymbol{j}^{-} \cdot \boldsymbol{n}+\rho a_{m}^{\perp} \omega^{-}=\mathcal{P}_{m}^{w}
$$

This relation can be interpreted in terms of a total water flux $\boldsymbol{j}_{\text {tot }}$ relative to the CTS velocity $\boldsymbol{w}$, defined by

$$
j_{\text {tot }}:=\rho_{w}\left(\boldsymbol{v}_{w}-\boldsymbol{w}\right),
$$

when (2.66) becomes

$$
-\boldsymbol{j}_{\text {tot }}^{-} \cdot \boldsymbol{n}=\mathcal{P}_{m}^{w}
$$

That is, the normal component of the total water flux relative to the CTS at the temperate side of the CTS equals the surface production of water.

To formulate the energy jump relation, as in the derivation of (2.22), the extended energy flux (2.21) for temperate ice is used, so that at the cold (positive) side $\boldsymbol{q}=\boldsymbol{q}_{s}$, and at the negative (temperate) side $\boldsymbol{q}=\boldsymbol{q}_{s}+L \boldsymbol{j}$. With (2.18), (2.63) and (2.64),

$$
\boldsymbol{q}_{s}^{+} \cdot \boldsymbol{n}-\boldsymbol{q}_{s}^{-} \cdot \boldsymbol{n}-L \boldsymbol{j}^{-} \cdot \boldsymbol{n}=L \omega^{-} \rho(\boldsymbol{v}-\boldsymbol{w}) \cdot \boldsymbol{n}=-L \omega^{-} \rho a_{m}^{\perp},
$$

or, with Fourier's heat conduction law and the definition of $\boldsymbol{j}_{\text {tot }}$,

$$
\kappa\left(\operatorname{grad} T^{+}-\operatorname{grad} T_{M}^{-}\right) \cdot \boldsymbol{n}+L \boldsymbol{j}_{\text {tot }}^{-} \cdot \boldsymbol{n}=0 .
$$

At the cold side of the CTS the homologous temperature $T^{\prime}=T-T_{M}$ cannot increase with the cold zone (otherwise the temperature would exceed the melting temperature); thus

$$
\operatorname{grad} T^{+} \cdot \boldsymbol{n}-\operatorname{grad} T_{M}^{-} \cdot \boldsymbol{n} \leq 0 .
$$

This and (2.70) imply $\boldsymbol{j}_{\text {tot }}^{-} \cdot \boldsymbol{n} \geq 0$, so that

$$
\mathcal{P}_{m}^{w} \quad\left(=-\boldsymbol{j}_{\text {tot }}^{-} \cdot \boldsymbol{n}\right) \quad \leq 0 ;
$$

that is, the surface production of water $\mathcal{P}_{m}^{w}$ cannot be positive.

Because of this secondary condition, for each point of the CTS three cases must be distinguished, depending on the sign of the quantity $\left(\boldsymbol{w}-\boldsymbol{v}_{w}^{-}\right) \cdot \boldsymbol{n}$ :

i) $\left(\boldsymbol{w}-\boldsymbol{v}_{w}^{-}\right) \cdot \boldsymbol{n}>0$ ("melting condition"):

With the above definition of $\boldsymbol{j}_{\text {tot }}(2.67)$ and $\rho_{w}=\rho \omega,(2.72)$ can only be fulfilled if

$$
\omega^{-}=0 ;
$$

so the equality in (2.72) holds. Inserting this into (2.70) yields further

$$
\operatorname{grad} T^{+} \cdot \boldsymbol{n}=\operatorname{grad} T_{M}^{-} \cdot \boldsymbol{n},
$$

which means that in case of melting conditions both the water content and the normal temperature derivative are continuous at the CTS ( $\omega^{+}$is equal to zero anyway, because at the cold side of the CTS by definition no water is present).

ii) $\left(\boldsymbol{w}-\boldsymbol{v}_{w}^{-}\right) \cdot \boldsymbol{n}<0$ ("freezing condition"):

In this case, $(2.72)$ is compatible with

$$
\omega^{-} \geq 0,
$$


so that $(2.71)$ can hold in its general form

$$
\operatorname{grad} T^{+} \cdot \boldsymbol{n} \leq \operatorname{grad} T_{M}^{-} \cdot \boldsymbol{n} .
$$

As a consequence, in case of freezing conditions the water content and the normal temperature derivative can be discontinuous at the CTS; the jumps of these quantities are connected by eq. (2.70).

iii) $\left(\boldsymbol{w}-\boldsymbol{v}_{w}^{-}\right) \cdot \boldsymbol{n}=0$ ("parallel-flow condition"):

For this case, too, (2.72) is compatible with

$$
\omega^{-} \geq 0
$$

however, equality holds automatically in (2.72). Inserting this into (2.70) provides

$$
\operatorname{grad} T^{+} \cdot \boldsymbol{n}=\operatorname{grad} T_{M}^{-} \cdot \boldsymbol{n} .
$$

Hence, the parallel-flow condition is characterized by a continuous normal temperature derivative (as for melting conditions), but the possibility of a jump in water content (as for freezing conditions).

This behaviour can be understood as follows: when a non-vanishing total water flux $\boldsymbol{j}_{\text {tot }}^{-}$reaches the CTS from the temperate side (freezing condition), the transported water can freeze at the CTS (negative surface production of water). The latent heat released can be conducted away by a negative normal temperature derivative in the cold zone exceeding the small negative gradient in the temperate zone. Therefore, this entails a jump of the normal temperature derivative and (since at the cold side the water content is zero) of the water content as well.

However, the opposite situation cannot occur: it is impossible that cold ice flows toward the CTS, melts partly at the CTS (positive surface production of water) and produces a non-vanishing total water flux at the temperate side. The reason for this is that the melting heat necessary for this process cannot be transported to the CTS; to achieve this the normal temperature derivative would have to be more positive at the cold side than at the temperate side, which is impossible because then the temperature in the cold zone would exceed the melting temperature of ice. Ice flow from the cold region through the CTS toward the temperate region is only possible without surface melting when passing the CTS, so that in this case $\omega^{-}=0$ and $\operatorname{grad} T^{+} \cdot \boldsymbol{n}=\operatorname{grad} T_{M}^{-} \cdot \boldsymbol{n}$ hold; in other words the water content and the normal temperature derivative are continuous.

It should further be mentioned that in the case of a negligible diffusive water flux $\boldsymbol{j}$ in temperate ice, that is, a very small water diffusivity $\nu$, the distinction between melting conditions, freezing conditions and parallel-flow conditions can simply be made by the sign of the ice volume flux through the CTS $a_{m}^{\perp}$, because in this case $\boldsymbol{v}=\boldsymbol{v}_{w}$ holds. $a_{m}^{\perp}>0$ (ice flow from cold to temperate ice) then corresponds to the melting condition, $a_{m}^{\perp}<0$ (ice flow from temperate to cold ice) to the freezing condition and $a_{m}^{\perp}=0$ to the parallel-flow condition.

\section{Ice sheet of uniform depth}

\subsection{Application of the model}

A simple application of the polythermal ice sheet model described above is now presented. Consider a two-dimensional inclined polythermal ice sheet of uniform depth ("slab"), infinitely extended in the $x$ direction, so the ice flows down the slope, as depicted in fig. 6. Numerical solutions for such a geometry have already been constructed by Hutter et. al. (1988) and Blatter (1991), but here a different approach provides essentially analytical solutions. As an explicit demonstration of the well-posed nature of the boundary value problem this is an essential mathematical step.

The following assumptions are made:

- Constant inclination angle $\gamma$, uniformity of the processes in $x$-direction: $(\partial / \partial x)(\cdot)=0$.

- Steady-state configuration: $(\partial / \partial t)(\cdot)=0$. 


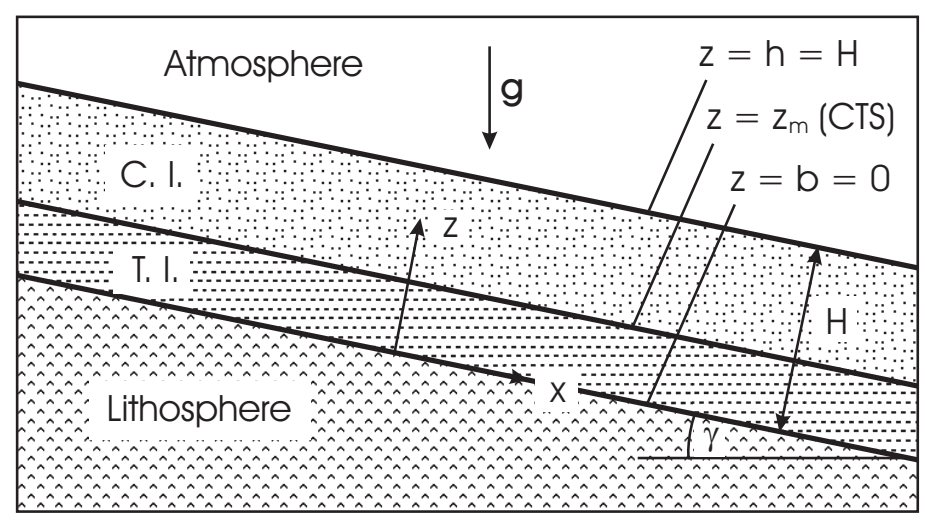

Figure 6: Ice sheet of uniform depth ("slab"): geometry and coordinate system. C. I.: cold ice, T. I.: temperate ice.

- Glen's flow law (cf. Glen, 1955; Nye, 1957; Hooke, 1981; Paterson, 1994): $f(\sigma)=f_{t}(\sigma)=\sigma^{n-1}($ with $n=3)$.

- Ice fluidity independent of temperature and water content: $E A\left(T^{\prime}\right)=E A_{t}(\omega) \equiv A=5.3 \cdot 10^{-24} \mathrm{~s}^{-1} \mathrm{~Pa}^{-3}$ (value for $T^{\prime}=0^{\circ} \mathrm{C}$ and $E=1$, see Paterson (1994)).

- $\rho=910 \mathrm{~kg} \mathrm{~m}^{-3}, \kappa=2.1 \mathrm{~W} \mathrm{~m}^{-1} \mathrm{~K}^{-1}, c=2009 \mathrm{~J} \mathrm{~kg}^{-1} \mathrm{~K}^{-1}, L=335 \mathrm{~kJ} \mathrm{~kg}^{-1}$, $g=9.81 \mathrm{~m} \mathrm{~s}^{-2}$.

- Neglect of acceleration: $\dot{\boldsymbol{v}}=\mathbf{0}$.

- Neglect of the pressure dependence of the melting point of ice: $T_{M} \equiv 0^{\circ} \mathrm{C}$.

- Neglect of water diffusion: $\nu=0 \Rightarrow \boldsymbol{j}=\mathbf{0}$.

- Neglect of lithospheric influences: no bedrock sinking, no calculation of lithosphere temperature and basal melting rate.

With these assumptions, the model equations become:

Mass balance, cold and temperate region (from eqs. (2.1), (2.13)):

$$
\frac{d v_{z}}{d z}=0
$$

Momentum balance, cold and temperate region (from eq. (2.3), (2.14)):

$$
\frac{d \sigma_{x z}}{d z}+\rho g \sin \gamma=0, \quad-\frac{d p}{d z}+\frac{d \sigma_{z}^{R}}{d z}-\rho g \cos \gamma=0
$$

Energy balance, cold region (from eq. (2.8)):

$$
\rho c v_{z} \frac{d T}{d z}=\kappa \frac{d^{2} T}{d z^{2}}+2 A \sigma^{4}
$$

Energy balance, temperate region; mass balance for the component water (from eqs. (2.23), (2.24)):

$$
\rho v_{z} \frac{d \omega}{d z}=2 \frac{A}{L} \sigma^{4}
$$


Stress-strain-rate relation, cold and temperate region (from eqs. (2.4), (2.17)):

$$
\sigma_{x}^{R}=0, \quad \sigma_{z}^{R}=0, \quad \frac{d v_{x}}{d z}=2 A \sigma^{2} \sigma_{x z}
$$

with the definition of the effective shear stress $\sigma:=\sqrt{\operatorname{tr}\left(\boldsymbol{T}^{R}\right)^{2} / 2}$ this yields $\sigma=\sigma_{x z}$.

Boundary conditions, cold free surface (from eqs. (2.33), (2.34), (2.35)):

$$
\begin{aligned}
v_{z} & =-a_{s}^{\perp}, & \sigma & =\sigma_{x z}=0, \\
T & =T_{s}, & -p+\sigma_{z}^{R} & =-p=0 .
\end{aligned}
$$

Boundary conditions, temperate base:

Due to eqs. (3.1) and (3.6), the velocity $v_{z}$ perpendicular to the bed equals the negative accumulationablation function $a_{s}^{\perp}$ for any vertical position in the slab. In particular, $v_{z}$ takes this value at the base as well, and as a consequence, the kinematic condition (2.50) is redundant. It determines the water mass flux into the base $\dot{m}_{b}^{w}$ required to sustain this basal $v_{z}$, but this does not affect the ice flow.

Boundary condition (2.51), which determines the normal diffusive water flux, is also redundant, since water diffusion is neglected. Thus, the sliding law (2.52) remains as the boundary condition for the basal velocity parallel to the bed, $v_{x, b}$. However, for simplicity, prescribe $v_{x, b}$ directly instead of formulating an explicit sliding law. The only impact of $v_{x, b}$ on the results is that it adds a constant to the velocity profile $v_{x}(z)$; temperature and water content are not affected at all.

Transition conditions, CTS (from eqs. (2.61), (2.63), (2.64), (2.69), (2.71)):

$$
\begin{aligned}
& T^{+}=T^{-}, \\
& v_{x}^{+}=v_{x}^{-}, \quad v_{z}^{+}=v_{z}^{-}, \\
& p^{+}=p^{-}, \quad \sigma_{(x z)}^{+}=\sigma_{(x z)}^{-}, \\
& \kappa \frac{d T^{+}}{d z}=L \omega^{-} \rho a_{m}^{\perp} \quad \text { with } \quad \frac{d T^{+}}{d z} \leq 0 .
\end{aligned}
$$

The secondary condition in the last equation entails that two different cases must be distinguished (see discussion in $\S 2.2 .5)$ :

- $a_{m}^{\perp}>0$ ("melting condition", ice flow from cold to temperate region): $d T^{+} / d z=0, \omega^{-}=0$.

- $a_{m}^{\perp}<0$ ("freezing condition", ice flow from temperate to cold region): Eq. (3.7) in its non-trivial form, i.e., $d T^{+} / d z$ can be strictly negative and $\omega^{-}$strictly positive; in this case an additional boundary condition for the basal water content is required.

The case of parallel flow is ignored, because it does not allow a steady-state solution. For this situation, because of (B.5) (see Appendix B) $v_{z} \equiv 0$ would hold, and thus the left-hand side of (3.4) would be zero, whereas its right-hand side would be strictly positive owing to (B.3). This contradiction proves therefore that steady parallel-flow conditions of this sort cannot occur.

\subsection{Results}

In Appendix B it is demonstrated how the slab equations compiled above can be solved semi-analytically. Here this solution is discussed for two cases, namely (i) melting conditions, and (ii) freezing conditions at the CTS. In detail, the parameters are: for case (i) thickness $H=200 \mathrm{~m}$, inclination angle $\gamma=4^{\circ}$, surface temperature $T_{s}=-3^{\circ} \mathrm{C}$, accumulation-ablation function $a_{s}^{\perp}=0.2 \mathrm{~m} \mathrm{a}^{-1}$ (i.e., $a_{m}^{\perp}=0.2 \mathrm{~m} \mathrm{a}^{-1}$ and $v_{z} \equiv-0.2 \mathrm{~m} \mathrm{a}^{-1}$ ), basal sliding velocity $v_{x, b}=5 \mathrm{ma}^{-1}$ (see fig. 7); and for case (ii) $H=200 \mathrm{~m}, \gamma=4^{\circ}$, $T_{s}=-10^{\circ} \mathrm{C}, a_{s}^{\perp}=-0.2 \mathrm{ma}^{-1}$ (i.e., $a_{m}^{\perp}=-0.2 \mathrm{~m} \mathrm{a}^{-1}$ and $v_{z} \equiv 0.2 \mathrm{~m} \mathrm{a}^{-1}$ ), $v_{x, b}=5 \mathrm{~m} \mathrm{a}^{-1}$ (see fig. 8).

In the corresponding figures panel (a) shows the velocity $v_{x}$ parallel to the bed, panel (b) the temperature $T$ in the cold region and the water content $\omega$ in the temperate region, all as functions of the vertical coordinate $z$. The distribution of the velocity $v_{x}$ is identical for both cases; it increases monotonically 


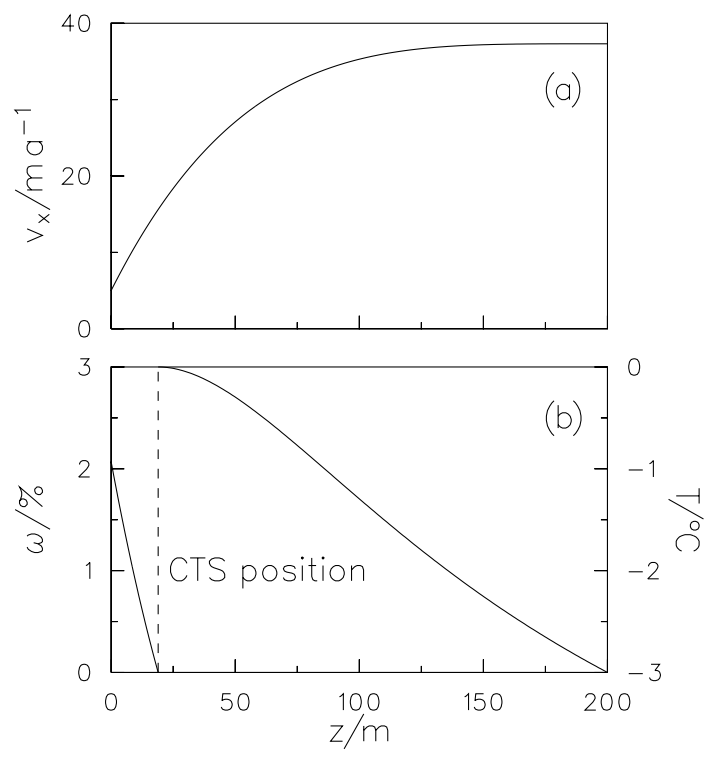

Figure 7: (a) Velocity $v_{x}$ parallel to the bed, (b) temperature $T$ and water content $\omega$, for the slab with melting conditions at the CTS. $H=200 \mathrm{~m}, \gamma=4^{\circ}, T_{s}=-3^{\circ} \mathrm{C}, a_{s}^{\perp}=0.2 \mathrm{~m} \mathrm{a}^{-1}, v_{x, b}=5 \mathrm{~m} \mathrm{a}^{-1}$.

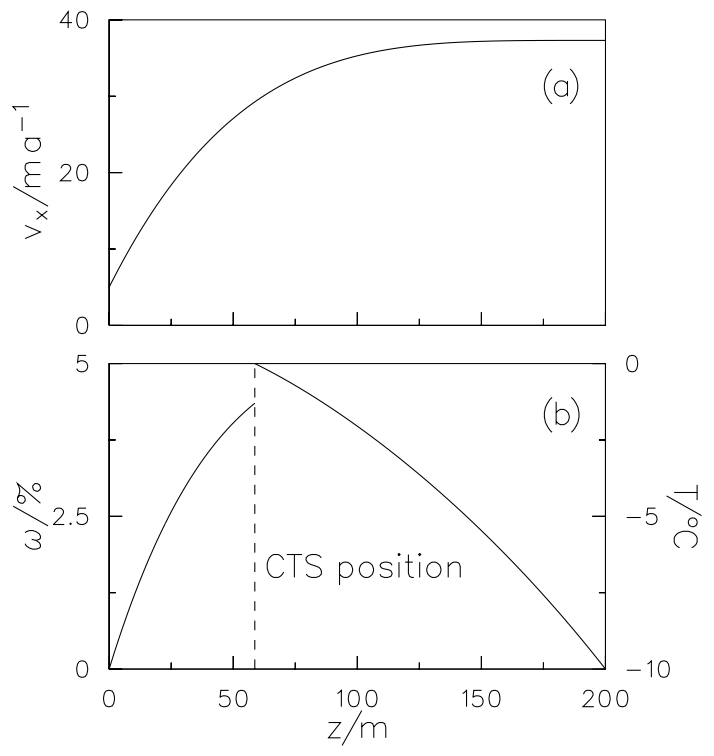

Figure 8: (a) Velocity $v_{x}$ parallel to the bed, (b) temperature $T$ and water content $\omega$, for the slab with freezing conditions at the CTS. $H=200 \mathrm{~m}, \gamma=4^{\circ}, T_{s}=-10^{\circ} \mathrm{C}, a_{s}^{\perp}=-0.2 \mathrm{~m} \mathrm{a}^{-1}, v_{x, b}=5 \mathrm{~m} \mathrm{a}^{-1}$.

from its minimum value at the base, as it is typical for such a shear flow problem. However, the behaviour of the temperature and the water content are entirely different: In the case of melting conditions (fig. 7) the temperature gradient $d T^{+} / d z$ at the cold side of the CTS and the water content at the temperate side vanish, as already explained in the discussion of eq. (3.7). Since these quantities are zero on the corresponding opposite sides of the CTS anyway, they are continuous and therefore do not jump. On the other hand, in the case of freezing conditions (fig. 8) a strictly negative temperature gradient $d T^{+} / d z$ and a strictly positive water content $\omega^{-}$appear, so that indeed there are discontinuities of these quantities. 
Finally, to anticipate a possible objection that this solution would not represent a realistic flow situation in a glacier or an ice sheet, note its real value in the following senses. First, the solution demonstrates that the model equations are well posed. Second, the solution is analytic (except for the determination of the CTS position by a Newtonian root finder), therefore exact, and can be used to check the performance of any numerical solution procedure.

\section{Scaling analysis and shallow-ice approximation}

The polythermal ice sheet model derived in $\S 2$ is in general still too complicated to be amenable to a numerical or analytical solution; only simple problems with strong symmetry (e.g., the ice sheet of uniform depth, see §3) can be analysed. Further simplification is therefore required. First, introduce an appropriate scaling in which the physical quantities are made dimensionless by the choice of corresponding typical magnitudes. Second, systematically neglect terms of small magnitude relative to the major balances. In this problem, the approximations are based on the assumption that a typical height scale in an ice sheet is much smaller than a typical length scale, and that respective gradients reflect this distinction in length scales. That is, the ice sheets are shallow ("shallow-ice approximation", in the following abbreviated by "SIA"; cf. Hutter, 1983; Morland, 1984; Blatter, 1991).

\subsection{Introduction of the scaling}

The various physical quantities will be scaled as follows:

$$
\begin{aligned}
& (x, y)=[L](\tilde{x}, \tilde{y}), \\
& z=[H] \tilde{z} \\
& \left(v_{x}, v_{y}\right)=\left[V_{L}\right]\left(\tilde{v}_{x}, \tilde{v}_{y}\right), \\
& v_{z}=\left[V_{H}\right] \tilde{v}_{z} \\
& t=\left([L] /\left[V_{L}\right]\right) \tilde{t}, \\
& \left(T, T^{\prime}\right)=[\Delta T]\left(\tilde{\theta}, \tilde{\theta}^{\prime}\right), \\
& \omega=[\omega] \tilde{\omega} \\
& p=\rho g[H] \tilde{p}, \\
& \left(\sigma_{x z}, \sigma_{y z}, \sigma\right)=\varepsilon \rho g[H]\left(\tilde{\sigma}_{x z}, \tilde{\sigma}_{y z}, \tilde{\sigma}\right), \\
& \left(\sigma_{x}^{R}, \sigma_{y}^{R}, \sigma_{z}^{R}\right)=\varepsilon^{2} \rho g[H]\left(\tilde{\sigma}_{x}^{R}, \tilde{\sigma}_{y}^{R}, \tilde{\sigma}_{z}^{R}\right), \\
& \sigma_{x y}=\varepsilon^{2} \rho g[\underset{\sim}{H}] \tilde{\sigma}_{x y}, \\
& \left(h, z_{m}, b, b_{r}\right)=[H]\left(\tilde{h}, \tilde{z}_{m}, \tilde{b}, \tilde{b}_{r}\right), \\
& \left(a_{s}^{\perp}, a_{m}^{\perp}\right)=\left[V_{H}\right]\left(\tilde{a}_{s}^{\perp}, \tilde{a}_{m}^{\perp}\right), \\
& \left(A\left(T^{\prime}\right), A_{t}(\omega)\right)=[A]\left(\tilde{A}\left(\tilde{\theta}^{\prime}\right), \tilde{A}_{t}(\tilde{\omega})\right), \\
& \left(f(\sigma), f_{t}(\sigma)\right)=[f]\left(\tilde{f}(\tilde{\sigma}), \tilde{f}_{t}(\tilde{\sigma})\right), \\
& Q_{\text {geoth }}^{\perp}=\left[Q_{\text {geoth }}^{\perp}\right] \tilde{Q}_{\text {geoth }}^{\perp}, \\
& \kappa(T)=[\kappa] \tilde{\kappa}(\tilde{\theta}) \\
& \kappa_{r}=\left[\kappa_{r}\right] \tilde{\kappa}_{r}, \\
& c(T)=[c] \tilde{c}(\tilde{\theta}), \\
& c_{r}=\left[c_{r}\right] \tilde{c}_{r}, \\
& C\left(t_{\perp}, \ldots\right)=[C] \tilde{C}\left(\tilde{t}_{\perp}, \ldots\right), \\
& C_{t}\left(t_{\perp}, \ldots\right)=\left[C_{t}\right] \tilde{C}_{t}\left(\tilde{t}_{\perp}, \ldots\right), \\
& \mathcal{P}_{b}^{w}=\rho[\omega]\left[V_{H}\right] \tilde{\mathcal{P}}_{b}^{w}, \\
& \dot{m}_{b}^{w}=\rho[\omega]\left[V_{H}\right] \tilde{\dot{m}}_{b}^{w}, \\
& \mathcal{P}_{m}^{w}=\rho[\omega]\left[V_{H}\right] \tilde{\mathcal{P}}_{m}^{w},
\end{aligned}
$$

with the aspect ratio $\varepsilon:=[H] /[L]=\left[V_{H}\right] /\left[V_{L}\right]$ (thus $\left[V_{H}\right]$ is not independent), and $T, T^{\prime}$ taken in ${ }^{\circ} \mathrm{C}$. Quantities in square brackets denote typical values for the respective variables, and variables marked with a tilde are dimensionless. Subsequently, unless stated otherwise, only dimensionless variables are used, and the tildes are omitted for simplicity.

The scalings are chosen such that the dimensionless quantities are all of order unity. This is evident for the pressure $p$ which reaches the overburden pressure magnitude $\rho g[H]$ at the base. The deviatoric stress scaling is chosen to satisfy the horizontal momentum balances when the dominant shear stresses are $\sigma_{x z}$ and $\sigma_{y z}$, which arises when basal friction is the dominant resistance to gravity-induced flow. This is appropriate to a grounded ice sheet as considered here, but not for a floating shelf where the basal shear traction supported by the water is negligible.

The 13 basic quantities introduced above are $[L],[H],\left[V_{L}\right],[\Delta T],[\omega],\left[Q_{\text {geoth }}^{\perp}\right],[\kappa],\left[\kappa_{r}\right],[c],\left[c_{r}\right],[C]$, $\left[C_{t}\right]$ and the product $[A][f]$ (neither $[A]$ nor $[f]$ appears otherwise), recalling that $\left[V_{H}\right]$ is not independent. Together with the eight physical constants, $\rho, g, L, \beta, \nu, \rho_{r}, \rho_{a}, \tau_{V}$, these form a set of 21 quantities, whose dimensions consist of the basic units meter, kilogram, second and Kelvin. The corresponding dimension 
matrix has rank four:

\begin{tabular}{c|rrrrr} 
& {$[L]$} & $\rho$ & {$\left[V_{L}\right]$} & {$[\Delta T]$} & $\ldots$ \\
\hline $\mathrm{m}$ & 1 & -3 & 1 & 0 & $\ldots$ \\
$\mathrm{kg}$ & 0 & 1 & 0 & 0 & $\ldots$ \\
$\mathrm{s}$ & 0 & 0 & -1 & 0 & $\ldots$ \\
$\mathrm{K}$ & 0 & 0 & 0 & 1 & $\ldots$
\end{tabular}

Due to the rules of dimensional analysis (Barenblatt, 1987), $13+8-4=17$ independent dimensionless products exist. A complete set of these products is

$$
\begin{array}{rlrlrl}
\varepsilon & =\frac{[H]}{[L]}=\frac{\left[V_{H}\right]}{\left[V_{L}\right]}, & \mathcal{D}_{t} & =\frac{\nu}{\rho[H]\left[V_{H}\right]}, & \mathcal{N}_{r}=\frac{[H]\left[Q_{\text {geoth }}\right]}{\left[\kappa_{r}\right][\Delta T]}, \\
\mathrm{F} & =\frac{\left[V_{L}\right]^{2}}{g[L]}, & \mathcal{F} & =\frac{\rho g[H]^{2}[C]}{[L]\left[V_{L}\right]}, & {[\omega],} \\
\mathcal{D} & =\frac{[\kappa]}{\rho[c][H]\left[V_{H}\right]}, & \mathcal{F}_{t} & =\frac{\rho g[H]^{2}\left[C_{t}\right]}{[L]\left[V_{L}\right]}, & \frac{\left[\kappa_{r}\right]}{[\kappa]}, \\
\alpha & =\frac{g[H]}{[c][\Delta T]}, & \mathcal{B} & =\frac{\beta[H]}{[\Delta T]}, \\
\mathcal{K} & =\frac{\rho g[H]^{3}[A][f]}{[L]\left[V_{L}\right]}, & \mathcal{D}_{r} & =\frac{\left[\kappa_{r}\right]}{\rho_{r}\left[c_{r}\right][H]\left[V_{H}\right]}, & \frac{\rho_{a}}{\rho} \\
\alpha_{t} & =\frac{g[H]}{L[\omega]}, & \mathcal{T}_{r} & =\frac{\tau_{V}\left[V_{H}\right]}{[H]},
\end{array}
$$

$\varepsilon$ is the aspect ratio, $\mathrm{F}$ the Froude number, $\mathcal{D}$ the heat diffusion number, $\alpha$ the ratio of potential energy to internal energy for cold ice, $\mathcal{K}$ the fluidity number, $\alpha_{t}$ the ratio of potential energy to internal energy for temperate ice, $\mathcal{D}_{t}$ the water diffusion number, $\mathcal{F}$ and $\mathcal{F}_{t}$ the sliding numbers for cold and temperate base, respectively, $\mathcal{B}$ the Clausius-Clapeyron number, $\mathcal{D}_{r}$ the heat diffusion number of the lithosphere, $\mathcal{T}_{r}$ the time-lag number for isostatic bed adjustment, and finally $\mathcal{N}_{r}$ the geothermal heat number in the lithosphere. For the last four combinations no special names are introduced.

\subsection{Scaling analysis and SIA for the model equations}

In the following all field equations, boundary and transition conditions of $\S 2$ are subjected to the above scaling, and subsequently simplified by the shallow-ice approximation (SIA). That is, in the limit

$$
\varepsilon \rightarrow 0
$$

all terms of order $O\left(\varepsilon^{p}\right)$ with $p \geq 1$ are neglected in comparison with unity (in real ice sheets, $\varepsilon$ is of the order $\left.10^{-3}\right)$. Furthermore, the Froude number in real ice sheets takes values of order $\mathrm{F}=10^{-15}$ or less. It is therefore meaningful to simultaneously apply the limit

$$
\frac{\mathrm{F}}{\varepsilon} \rightarrow 0
$$

\subsubsection{Cold regions}

The scaled mass balance from (2.1) becomes

$$
\frac{\partial v_{x}}{\partial x}+\frac{\partial v_{y}}{\partial y}+\frac{\partial v_{z}}{\partial z}=0
$$

recalling that tildes are now omitted, which cannot be simplified further by the SIA. The scaled momentum balance $(2.3)$ is

$$
\frac{\mathrm{F}}{\varepsilon} \frac{d v_{x}}{d t}=-\frac{\partial p}{\partial x}+\varepsilon^{2} \frac{\partial \sigma_{x}^{R}}{\partial x}+\varepsilon^{2} \frac{\partial \sigma_{x y}}{\partial y}+\frac{\partial \sigma_{x z}}{\partial z}
$$




$$
\begin{aligned}
& \frac{\mathrm{F}}{\varepsilon} \frac{d v_{y}}{d t}=\varepsilon^{2} \frac{\partial \sigma_{x y}}{\partial x}-\frac{\partial p}{\partial y}+\varepsilon^{2} \frac{\partial \sigma_{y}^{R}}{\partial y}+\frac{\partial \sigma_{y z}}{\partial z} \\
& \mathrm{~F} \varepsilon \frac{d v_{z}}{d t}=\varepsilon^{2} \frac{\partial \sigma_{x z}}{\partial x}+\varepsilon^{2} \frac{\partial \sigma_{y z}}{\partial y}-\frac{\partial p}{\partial z}+\varepsilon^{2} \frac{\partial \sigma_{z}^{R}}{\partial z}-1
\end{aligned}
$$

Application of the SIA yields

$$
\begin{aligned}
-\frac{\partial p}{\partial x}+\frac{\partial \sigma_{x z}}{\partial z} & =0 \\
-\frac{\partial p}{\partial y}+\frac{\partial \sigma_{y z}}{\partial z} & =0 \\
-\frac{\partial p}{\partial z} & =1
\end{aligned}
$$

which is an impressive example of how much the general equations of $\S 2$ are simplified by the SIA. The result shows further that the scaling of the shear stresses $\sigma_{x z}$ and $\sigma_{y z}$ with $\varepsilon \rho g[H]$ (see $\S 4.1$ ) is appropriate, because without the additional $\varepsilon$ compared to the pressure scaling, eqs. (4.9) and (4.10) would read $\partial \sigma_{x z} / \partial z=0, \partial \sigma_{y z} / \partial z=0$, a non-physical result for a free-surface flow governed by gravity and basal friction (see Morland and Johnsen, 1980; Hutter, 1983; Morland, 1984).

The energy balance (2.8) becomes with an explicitly written advection term

$$
\begin{aligned}
& \frac{\partial \theta}{\partial t}+v_{x} \frac{\partial \theta}{\partial x}+v_{y} \frac{\partial \theta}{\partial y}+v_{z} \frac{\partial \theta}{\partial z}=\frac{\mathcal{D}}{c}\left\{\varepsilon^{2} \frac{\partial}{\partial x}\left(\kappa \frac{\partial \theta}{\partial x}\right)\right. \\
& \left.+\varepsilon^{2} \frac{\partial}{\partial y}\left(\kappa \frac{\partial \theta}{\partial y}\right)+\frac{\partial}{\partial z}\left(\kappa \frac{\partial \theta}{\partial z}\right)\right\}+2 \frac{\alpha}{c} \mathcal{K} E A\left(\theta^{\prime}\right) f(\sigma) \sigma^{2},
\end{aligned}
$$

and in the SIA limit,

$$
\frac{\partial \theta}{\partial t}+v_{x} \frac{\partial \theta}{\partial x}+v_{y} \frac{\partial \theta}{\partial y}+v_{z} \frac{\partial \theta}{\partial z}=\frac{\mathcal{D}}{c} \frac{\partial}{\partial z}\left(\kappa \frac{\partial \theta}{\partial z}\right)+2 \frac{\alpha}{c} \mathcal{K} E A\left(\theta^{\prime}\right) f(\sigma) \sigma^{2} .
$$

Horizontal heat conduction can therefore be neglected in the SIA limit, but horizontal, as well as vertical, advection must be kept.

The stress-strain-rate relation (2.4) takes the component form

$$
\begin{aligned}
\frac{\partial v_{x}}{\partial x} & =\mathcal{K} E A\left(\theta^{\prime}\right) f(\sigma) \sigma_{x}^{R}, \\
\frac{\partial v_{y}}{\partial y} & =\mathcal{K} E A\left(\theta^{\prime}\right) f(\sigma) \sigma_{y}^{R}, \\
\frac{\partial v_{z}}{\partial z} & =\mathcal{K} E A\left(\theta^{\prime}\right) f(\sigma) \sigma_{z}^{R}, \\
\frac{\partial v_{x}}{\partial y}+\frac{\partial v_{y}}{\partial x} & =2 \mathcal{K} E A\left(\theta^{\prime}\right) f(\sigma) \sigma_{x y}, \\
\frac{\partial v_{x}}{\partial z}\left\{+\varepsilon^{2} \frac{\partial v_{z}}{\partial x}\right\} & =2 \mathcal{K} E A\left(\theta^{\prime}\right) f(\sigma) \sigma_{x z}, \\
\frac{\partial v_{y}}{\partial z}\left\{+\varepsilon^{2} \frac{\partial v_{z}}{\partial y}\right\} & =2 \mathcal{K} E A\left(\theta^{\prime}\right) f(\sigma) \sigma_{y z},
\end{aligned}
$$

and with the SIA the $O\left(\varepsilon^{2}\right)$ terms in curly brackets vanish. This makes clear that the scaling of the normal frictional stresses $\sigma_{x}^{R}, \sigma_{y}^{R}, \sigma_{z}^{R}$ and of the shear stress $\sigma_{x y}$ with $\varepsilon^{2} \rho g[H]$, according to $\S 4.1$, is appropriate, since it provides order-consistent terms in all equations. These stresses are therefore very small in shallow grounded ice sheets. Of the above relations only (4.18) and (4.19) will be needed to construct the leading order stresses. Moreover, the effective shear stress (required for the energy balance and for the creep 
response function),

$$
\sigma=\sqrt{\sigma_{x z}^{2}+\sigma_{y z}^{2}+\varepsilon^{2}\left(\frac{\left(\sigma_{x}^{R}\right)^{2}}{2}+\frac{\left(\sigma_{y}^{R}\right)^{2}}{2}+\frac{\left(\sigma_{z}^{R}\right)^{2}}{2}+\sigma_{x y}^{2}\right)},
$$

simplifies in the SIA limit to

$$
\sigma=\sqrt{\sigma_{x z}^{2}+\sigma_{y z}^{2}}
$$

\subsubsection{Temperate regions}

As already stated, the mixture mass balance and the mixture momentum balance for ice plus water have the same form as the corresponding balances for cold ice (see (2.1), (2.3), (2.13), (2.14)); therefore, the scaled and SIA versions are the same as in the previous section (eqs. (4.5) - (4.11)), and will not be listed again.

For the temperature, according to $(2.9)$,

$$
\theta=\theta_{M}=-\mathcal{B} p
$$

For the mass balance of the component water (2.23) and the mixture energy balance (2.24), which are the same provided that (2.25) is applied, we obtain

$$
\begin{aligned}
& \frac{\partial \omega}{\partial t}+v_{x} \frac{\partial \omega}{\partial x}+v_{y} \frac{\partial \omega}{\partial y}+v_{z} \frac{\partial \omega}{\partial z}+\frac{c \alpha_{t}}{\alpha}\left(\frac{\partial \theta_{M}}{\partial t}+v_{x} \frac{\partial \theta_{M}}{\partial x}+v_{y} \frac{\partial \theta_{M}}{\partial y}+v_{z} \frac{\partial \theta_{M}}{\partial z}\right) \\
& =\mathcal{D}_{t}\left(\varepsilon^{2} \frac{\partial^{2} \omega}{\partial x^{2}}+\varepsilon^{2} \frac{\partial^{2} \omega}{\partial y^{2}}+\frac{\partial^{2} \omega}{\partial z^{2}}\right) \\
& +\frac{\mathcal{D} \alpha_{t}}{\alpha}\left\{\varepsilon^{2} \frac{\partial}{\partial x}\left(\kappa \frac{\partial \theta_{M}}{\partial x}\right)+\varepsilon^{2} \frac{\partial}{\partial y}\left(\kappa \frac{\partial \theta_{M}}{\partial y}\right)+\frac{\partial}{\partial z}\left(\kappa \frac{\partial \theta_{M}}{\partial z}\right)\right\} \\
& +2 \alpha_{t} \mathcal{K} E A_{t}(\omega) f_{t}(\sigma) \sigma^{2},
\end{aligned}
$$

which in the SIA reduces to

$$
\begin{aligned}
& \frac{\partial \omega}{\partial t}+v_{x} \frac{\partial \omega}{\partial x}+v_{y} \frac{\partial \omega}{\partial y}+v_{z} \frac{\partial \omega}{\partial z}+\frac{c \alpha_{t}}{\alpha}\left(\frac{\partial \theta_{M}}{\partial t}+v_{x} \frac{\partial \theta_{M}}{\partial x}+v_{y} \frac{\partial \theta_{M}}{\partial y}+v_{z} \frac{\partial \theta_{M}}{\partial z}\right) \\
& =\mathcal{D}_{t} \frac{\partial^{2} \omega}{\partial z^{2}}+\frac{\mathcal{D} \alpha_{t}}{\alpha} \frac{\partial}{\partial z}\left(\kappa \frac{\partial \theta_{M}}{\partial z}\right)+2 \alpha_{t} \mathcal{K} E A_{t}(\omega) f_{t}(\sigma) \sigma^{2}
\end{aligned}
$$

As was the case for the energy balance of cold ice, horizontal diffusion is neglected.

The stress-strain-rate relation (2.17) is basically the same as for cold ice (2.4). Hence eqs. (4.14) - (4.19) also hold for temperate ice, merely $A\left(\theta^{\prime}\right)$ and $f(\sigma)$ must be replaced by $A_{t}(\omega)$ and $f_{t}(\sigma)$, respectively. Also the result for the effective shear stress remains unchanged.

\subsubsection{Lithosphere}

The scaled energy balance (2.26)

$$
\frac{\partial \theta}{\partial t}+v_{x} \frac{\partial \theta}{\partial x}+v_{y} \frac{\partial \theta}{\partial y}+v_{z} \frac{\partial \theta}{\partial z}=\frac{\mathcal{D}_{r} \kappa_{r}}{c_{r}}\left(\varepsilon^{2} \frac{\partial^{2} \theta}{\partial x^{2}}+\varepsilon^{2} \frac{\partial^{2} \theta}{\partial y^{2}}+\frac{\partial^{2} \theta}{\partial z^{2}}\right)
$$

reduces in the SIA limit to

$$
\frac{\partial \theta}{\partial t}+v_{x} \frac{\partial \theta}{\partial x}+v_{y} \frac{\partial \theta}{\partial y}+v_{z} \frac{\partial \theta}{\partial z}=\frac{\mathcal{D}_{r} \kappa_{r}}{c_{r}} \frac{\partial^{2} \theta}{\partial z^{2}}
$$

The evolution of the lithosphere surface follows from (2.29) as

$$
\frac{\partial b}{\partial t}=-\frac{1}{\mathcal{T}_{r}}\left[b-\left(b_{0}-\frac{\rho}{\rho_{a}} H\right)\right]
$$


this cannot be further simplified. Correspondingly, the lithosphere velocity simplifies to

$$
v_{x}=0, \quad v_{y}=0, \quad v_{z}=\frac{\partial b}{\partial t}(x, y, t)
$$

according to (2.30), with $\partial b / \partial t$ from equation (4.27).

\subsubsection{Boundary conditions at the free surface}

The scaled kinematic condition (2.33) is ${ }^{2}$

$$
\frac{\partial h}{\partial t}+v_{x} \frac{\partial h}{\partial x}+v_{y} \frac{\partial h}{\partial y}-v_{z}=\left(1+\varepsilon^{2}\left(\frac{\partial h}{\partial x}\right)^{2}+\varepsilon^{2}\left(\frac{\partial h}{\partial y}\right)^{2}\right)^{1 / 2} a_{s}^{\perp},
$$

which in the SIA becomes

$$
\frac{\partial h}{\partial t}+v_{x} \frac{\partial h}{\partial x}+v_{y} \frac{\partial h}{\partial y}-v_{z}=a_{s}^{\perp} .
$$

For subsequent developments the terms

$$
\begin{aligned}
\operatorname{grad} F_{s} & =\left(-\varepsilon \frac{\partial h}{\partial x},-\varepsilon \frac{\partial h}{\partial y}, 1\right)^{t} \\
\left\|\operatorname{grad} F_{s}\right\| & =\left(1+\varepsilon^{2}\left(\frac{\partial h}{\partial x}\right)^{2}+\varepsilon^{2}\left(\frac{\partial h}{\partial y}\right)^{2}\right)^{1 / 2}
\end{aligned}
$$

are required, and in the SIA limit they reduce to

$$
\operatorname{grad} F_{s}=(0,0,1)^{t}, \quad\left\|\operatorname{grad} F_{s}\right\|=1 .
$$

With the outer normal unit vector $\boldsymbol{n}=\operatorname{grad} F_{s} /\left\|\operatorname{grad} F_{s}\right\|$, the component forms of the momentum jump relation (2.34) are

$$
\begin{aligned}
&-\left(-p+\varepsilon^{2} \sigma_{x}^{R}\right) \frac{\partial h}{\partial x}-\varepsilon^{2} \sigma_{x y} \frac{\partial h}{\partial y}+\sigma_{x z}=0 \\
&-\varepsilon^{2} \sigma_{x y} \frac{\partial h}{\partial x}-\left(-p+\varepsilon^{2} \sigma_{y}^{R}\right) \frac{\partial h}{\partial y}+\sigma_{y z}=0 \\
&-\varepsilon^{2} \sigma_{x z} \frac{\partial h}{\partial x}-\varepsilon^{2} \sigma_{y z} \frac{\partial h}{\partial y}-p+\varepsilon^{2} \sigma_{z}^{R}=0 .
\end{aligned}
$$

Application of the SIA first simplifies the last of these equations to

$$
p=0,
$$

and with this, the first two equations yield

$$
\sigma_{x z}=0, \quad \sigma_{y z}=0 .
$$

In the case of a cold free surface there remains the boundary condition for the surface temperature (2.35), which is

$$
\theta(x, y, t)=\theta_{s}(x, y, t) .
$$

In the case of a temperate free surface, the water content $\omega$ or its normal derivative must be prescribed.

\footnotetext{
${ }^{2}$ The minus-superscripts $(\cdot)^{-}$that mark the ice side in the boundary conditions at the free surface as well as in the transition conditions at the ice base (see $\S 2)$ are omitted in the following. Therefore, unmarked quantities always refer to the ice side.
} 


\subsubsection{Transition conditions at the cold ice base}

The kinematic condition (2.38) remains formally unchanged under scaling and the SIA,

$$
\frac{\partial b}{\partial t}+v_{x} \frac{\partial b}{\partial x}+v_{y} \frac{\partial b}{\partial y}-v_{z}=0
$$

Analogous to the free surface,

$$
\begin{aligned}
\operatorname{grad} F_{b} & =\left(\varepsilon \frac{\partial b}{\partial x}, \varepsilon \frac{\partial b}{\partial y},-1\right)^{t}, \\
\left\|\operatorname{grad} F_{b}\right\| & =\left(1+\varepsilon^{2}\left(\frac{\partial b}{\partial x}\right)^{2}+\varepsilon^{2}\left(\frac{\partial b}{\partial y}\right)^{2}\right)^{1 / 2},
\end{aligned}
$$

and in the SIA limit,

$$
\operatorname{grad} F_{b}=(0,0,-1)^{t}, \quad\left\|\operatorname{grad} F_{b}\right\|=1
$$

With $\boldsymbol{n}=\operatorname{grad} F_{b} /\left\|\operatorname{grad} F_{b}\right\|$,

$$
\boldsymbol{T n}=\frac{\rho g[H]}{\left\|\operatorname{grad} F_{b}\right\|}\left(\begin{array}{c}
\varepsilon \sigma_{x} \frac{\partial b}{\partial x}+\varepsilon^{3} \sigma_{x y} \frac{\partial b}{\partial y}-\varepsilon \sigma_{x z} \\
\varepsilon^{3} \sigma_{x y} \frac{\partial b}{\partial x}+\varepsilon \sigma_{y} \frac{\partial b}{\partial y}-\varepsilon \sigma_{y z} \\
\varepsilon^{2} \sigma_{x z} \frac{\partial b}{\partial x}+\varepsilon^{2} \sigma_{y z} \frac{\partial b}{\partial y}-\sigma_{z}
\end{array}\right),
$$

and

$$
\begin{aligned}
& (\boldsymbol{n} \cdot \boldsymbol{T n}) \boldsymbol{n}=\frac{\rho g[H]}{\left\|\operatorname{grad} F_{b}\right\|^{3}} \times \\
& \left(\begin{array}{l}
\varepsilon^{3} \sigma_{x}\left(\frac{\partial b}{\partial x}\right)^{3}+\varepsilon^{3} \sigma_{y} \frac{\partial b}{\partial x}\left(\frac{\partial b}{\partial y}\right)^{2}+\varepsilon \sigma_{z} \frac{\partial b}{\partial x} \\
+2 \varepsilon^{5} \sigma_{x y}\left(\frac{\partial b}{\partial x}\right)^{2} \frac{\partial b}{\partial y}-2 \varepsilon^{3} \sigma_{x z}\left(\frac{\partial b}{\partial x}\right)^{2}-2 \varepsilon^{3} \sigma_{y z} \frac{\partial b}{\partial x} \frac{\partial b}{\partial y} \\
\varepsilon^{3} \sigma_{x}\left(\frac{\partial b}{\partial x}\right)^{2} \frac{\partial b}{\partial y}+\varepsilon^{3} \sigma_{y}\left(\frac{\partial b}{\partial y}\right)^{3}+\varepsilon \sigma_{z} \frac{\partial b}{\partial y} \\
+2 \varepsilon^{5} \sigma_{x y} \frac{\partial b}{\partial x}\left(\frac{\partial b}{\partial y}\right)^{2}-2 \varepsilon^{3} \sigma_{x z} \frac{\partial b}{\partial x} \frac{\partial b}{\partial y}-2 \varepsilon^{3} \sigma_{y z}\left(\frac{\partial b}{\partial y}\right)^{2} \\
-\varepsilon^{2} \sigma_{x}\left(\frac{\partial b}{\partial x}\right)^{2}-\varepsilon^{2} \sigma_{y}\left(\frac{\partial b}{\partial y}\right)^{2}-\sigma_{z} \\
-2 \varepsilon^{4} \sigma_{x y} \frac{\partial b}{\partial x} \frac{\partial b}{\partial y}+2 \varepsilon^{2} \sigma_{x z} \frac{\partial b}{\partial x}+2 \varepsilon^{2} \sigma_{y z} \frac{\partial b}{\partial y}
\end{array}\right),
\end{aligned}
$$

the sliding law (2.39) becomes

$$
\begin{aligned}
\left(v_{\mathrm{sl}}\right)_{x}= & -\frac{\mathcal{F} C}{\left\|\operatorname{grad} F_{b}\right\|}\left[\sigma_{x} \frac{\partial b}{\partial x}+\varepsilon^{2} \sigma_{x y} \frac{\partial b}{\partial y}-\sigma_{x z}\right. \\
& -\frac{1}{\left\|\operatorname{grad} F_{b}\right\|^{2}}\left(\varepsilon^{2} \sigma_{x}\left(\frac{\partial b}{\partial x}\right)^{3}+\varepsilon^{2} \sigma_{y} \frac{\partial b}{\partial x}\left(\frac{\partial b}{\partial y}\right)^{2}+\sigma_{z} \frac{\partial b}{\partial x}\right. \\
& \left.\left.+2 \varepsilon^{4} \sigma_{x y}\left(\frac{\partial b}{\partial x}\right)^{2} \frac{\partial b}{\partial y}-2 \varepsilon^{2} \sigma_{x z}\left(\frac{\partial b}{\partial x}\right)^{2}-2 \varepsilon^{2} \sigma_{y z} \frac{\partial b}{\partial x} \frac{\partial b}{\partial y}\right)\right],
\end{aligned}
$$




$$
\begin{aligned}
\left(v_{\mathrm{sl}}\right)_{y}= & -\frac{\mathcal{F} C}{\left\|\operatorname{grad} F_{b}\right\|}\left[\varepsilon^{2} \sigma_{x y} \frac{\partial b}{\partial x}+\sigma_{y} \frac{\partial b}{\partial y}-\sigma_{y z}\right. \\
& -\frac{1}{\left\|\operatorname{grad} F_{b}\right\|^{2}}\left(\varepsilon^{2} \sigma_{x}\left(\frac{\partial b}{\partial x}\right)^{2} \frac{\partial b}{\partial y}+\varepsilon^{2} \sigma_{y}\left(\frac{\partial b}{\partial y}\right)^{3}+\sigma_{z} \frac{\partial b}{\partial y}\right. \\
& \left.\left.+2 \varepsilon^{4} \sigma_{x y} \frac{\partial b}{\partial x}\left(\frac{\partial b}{\partial y}\right)^{2}-2 \varepsilon^{2} \sigma_{x z} \frac{\partial b}{\partial x} \frac{\partial b}{\partial y}-2 \varepsilon^{2} \sigma_{y z}\left(\frac{\partial b}{\partial y}\right)^{2}\right)\right], \\
\left(v_{\mathrm{sl}}\right)_{z}= & -\frac{\mathcal{F} C}{\left\|\operatorname{grad} F_{b}\right\|}\left[\sigma_{x z} \frac{\partial b}{\partial x}+\sigma_{y z} \frac{\partial b}{\partial y}-\frac{\sigma_{z}}{\varepsilon^{2}}\right. \\
& -\frac{1}{\left\|\operatorname{grad} F_{b}\right\|^{2}}\left(-\sigma_{x}\left(\frac{\partial b}{\partial x}\right)^{2}-\sigma_{y}\left(\frac{\partial b}{\partial y}\right)^{2}-\frac{\sigma_{z}}{\varepsilon^{2}}\right. \\
& \left.\left.-2 \varepsilon^{2} \sigma_{x y} \frac{\partial b}{\partial x} \frac{\partial b}{\partial y}+2 \sigma_{x z} \frac{\partial b}{\partial x}+2 \sigma_{y z} \frac{\partial b}{\partial y}\right)\right] .
\end{aligned}
$$

In view of $1 /\left\|\operatorname{grad} F_{b}\right\|^{2}=1-\varepsilon^{2}(\partial b / \partial x)^{2}-\varepsilon^{2}(\partial b / \partial y)^{2}+O\left(\varepsilon^{4}\right)$ (noting that eq. (4.48) contains $O\left(\varepsilon^{-2}\right)$ terms, the two terms of order $O\left(\varepsilon^{2}\right)$ are relevant), the SIA reductions are

$$
\begin{aligned}
\left(v_{\mathrm{sl}}\right)_{x} & =\mathcal{F} C \sigma_{x z}, \\
\left(v_{\mathrm{sl}}\right)_{y} & =\mathcal{F} C \sigma_{y z}, \\
\left(v_{\mathrm{sl}}\right)_{z} & =\mathcal{F} C\left(\frac{\partial b}{\partial x} \sigma_{x z}+\frac{\partial b}{\partial y} \sigma_{y z}\right) .
\end{aligned}
$$

According to its definition (see eq. (2.38)), the sliding velocity $\boldsymbol{v}_{\mathrm{sl}}$ can be expressed as

$$
\begin{aligned}
\left(v_{\mathrm{sl}}\right)_{x}= & v_{x}-v_{x}^{+}-\frac{\varepsilon^{2}}{\left\|\operatorname{grad} F_{b}\right\|^{2}} \times \\
& \left(\left(v_{x}-v_{x}^{+}\right) \frac{\partial b}{\partial x}+\left(v_{y}-v_{y}^{+}\right) \frac{\partial b}{\partial y}-\left(v_{z}-v_{z}^{+}\right)\right) \frac{\partial b}{\partial x}, \\
\left(v_{\mathrm{sl}}\right)_{y}= & v_{y}-v_{y}^{+}-\frac{\varepsilon^{2}}{\left\|\operatorname{grad} F_{b}\right\|^{2}} \times \\
& \left(\left(v_{x}-v_{x}^{+}\right) \frac{\partial b}{\partial x}+\left(v_{y}-v_{y}^{+}\right) \frac{\partial b}{\partial y}-\left(v_{z}-v_{z}^{+}\right)\right) \frac{\partial b}{\partial y}, \\
\left(v_{\mathrm{sl}}\right)_{z}= & v_{z}-v_{z}^{+}+\frac{1}{\left\|\operatorname{grad} F_{b}\right\|^{2}} \times \\
& \left(\left(v_{x}-v_{x}^{+}\right) \frac{\partial b}{\partial x}+\left(v_{y}-v_{y}^{+}\right) \frac{\partial b}{\partial y}-\left(v_{z}-v_{z}^{+}\right)\right),
\end{aligned}
$$

and application of the SIA limit yields

$$
\begin{aligned}
\left(v_{\mathrm{sl}}\right)_{x} & =v_{x}-v_{x}^{+}, \\
\left(v_{\mathrm{sl}}\right)_{y} & =v_{y}-v_{y}^{+}, \\
\left(v_{\mathrm{sl}}\right)_{z} & =\left(v_{x}-v_{x}^{+}\right) \frac{\partial b}{\partial x}+\left(v_{y}-v_{y}^{+}\right) \frac{\partial b}{\partial y} .
\end{aligned}
$$

Further, for the lithosphere model applied here, $v_{x}^{+}$and $v_{y}^{+}$vanish; see eqs. (4.28).

The momentum jump relation (2.40) is not required, since the lithosphere stresses are not calculated.

The energy jump relation (2.41) in scaled form is

$$
\kappa\left(\varepsilon^{2} \frac{\partial \theta}{\partial x} \frac{\partial b}{\partial x}+\varepsilon^{2} \frac{\partial \theta}{\partial y} \frac{\partial b}{\partial y}-\frac{\partial \theta}{\partial z}\right)-\frac{\left[\kappa_{r}\right]}{[\kappa]} \kappa_{r}\left(\varepsilon^{2} \frac{\partial \theta^{+}}{\partial x} \frac{\partial b}{\partial x}+\varepsilon^{2} \frac{\partial \theta^{+}}{\partial y} \frac{\partial b}{\partial y}-\frac{\partial \theta^{+}}{\partial z}\right)
$$




$$
\begin{aligned}
& =-\frac{\alpha}{\mathcal{D}}\left\{\left(v_{\mathrm{sl}}\right)_{x} \frac{\partial b}{\partial x}\left(-p+\varepsilon^{2} \sigma_{x}^{R}\right)+\varepsilon^{2}\left(v_{\mathrm{sl}}\right)_{x} \frac{\partial b}{\partial y} \sigma_{x y}-\left(v_{\mathrm{sl}}\right)_{x} \sigma_{x z}\right. \\
& +\varepsilon^{2}\left(v_{\mathrm{sl}}\right)_{y} \frac{\partial b}{\partial x} \sigma_{x y}+\left(v_{\mathrm{sl}}\right)_{y} \frac{\partial b}{\partial y}\left(-p+\varepsilon^{2} \sigma_{y}^{R}\right)-\left(v_{\mathrm{sl}}\right)_{y} \sigma_{y z} \\
& \left.+\varepsilon^{2}\left(v_{\mathrm{sl}}\right)_{z} \frac{\partial b}{\partial x} \sigma_{x z}+\varepsilon^{2}\left(v_{\mathrm{sl}}\right)_{z} \frac{\partial b}{\partial y} \sigma_{y z}-\left(v_{\mathrm{sl}}\right)_{z}\left(-p+\varepsilon^{2} \sigma_{z}^{R}\right)\right\} .
\end{aligned}
$$

If adhesion at the cold base is assumed, the term $-(\alpha / \mathcal{D})\{\ldots\}$ vanishes. The SIA limit of $(4.58)$ is

$$
\kappa \frac{\partial \theta}{\partial z}-\frac{\left[\kappa_{r}\right]}{[\kappa]} \kappa_{r} \frac{\partial \theta^{+}}{\partial z}=-\frac{\alpha}{\mathcal{D}}\left[\left(v_{\mathrm{sl}}\right)_{x} \sigma_{x z}+\left(v_{\mathrm{sl}}\right)_{y} \sigma_{y z}\right] ;
$$

the terms containing the pressure $p$ in (4.58) vanish due to eqs. $(4.55)-(4.57)$.

Finally, there is the continuity of the temperature (eq. (2.42)),

$$
\theta=\theta^{+} \text {. }
$$

\subsubsection{Transition conditions at the temperate ice base}

The relations $(4.41)-(4.43)$ for $\operatorname{grad} F_{b}$ and $\left\|\operatorname{grad} F_{b}\right\|$ are valid for this case as well. Thus, the kinematic condition (2.50) is

$$
\frac{\partial b}{\partial t}+v_{x} \frac{\partial b}{\partial x}+v_{y} \frac{\partial b}{\partial y}-v_{z}=\left(1+\varepsilon^{2}\left(\frac{\partial b}{\partial x}\right)^{2}+\varepsilon^{2}\left(\frac{\partial b}{\partial y}\right)^{2}\right)^{1 / 2}[\omega] \dot{m}_{b}^{w}
$$

and in the SIA limit,

$$
\frac{\partial b}{\partial t}+v_{x} \frac{\partial b}{\partial x}+v_{y} \frac{\partial b}{\partial y}-v_{z}=[\omega] \dot{m}_{b}^{w}
$$

The boundary condition for the water content (2.51),

$$
\frac{\mathcal{D}_{t}}{\left\|\operatorname{grad} F_{b}\right\|}\left(-\varepsilon^{2} \frac{\partial \omega}{\partial x} \frac{\partial b}{\partial x}-\varepsilon^{2} \frac{\partial \omega}{\partial y} \frac{\partial b}{\partial y}+\frac{\partial \omega}{\partial z}\right)=(1-[\omega] \omega) \dot{m}_{b}^{w}-\mathcal{P}_{b}^{w},
$$

reduces in the SIA limit to

$$
\mathcal{D}_{t} \frac{\partial \omega}{\partial z}=(1-[\omega] \omega) \dot{m}_{b}^{w}-\mathcal{P}_{b}^{w} .
$$

Scaling of the sliding law (2.52) is carried out analogous to the procedure for a cold ice base, simply replacing $\mathcal{F} C$ by $\mathcal{F}_{t} C_{t}$.

The energy jump relation (2.56) yields

$$
\begin{aligned}
\mathcal{P}_{b}^{w}= & \frac{\mathcal{D} \alpha_{t}}{\alpha} \frac{\kappa}{\left\|\operatorname{grad} F_{b}\right\|}\left\{-\varepsilon^{2} \frac{\partial \theta}{\partial x} \frac{\partial b}{\partial x}-\varepsilon^{2} \frac{\partial \theta}{\partial y} \frac{\partial b}{\partial y}+\frac{\partial \theta}{\partial z}\right\} \\
& -\frac{\mathcal{D} \alpha_{t}}{\alpha} \frac{\left[\kappa_{r}\right]}{[\kappa]} \frac{\kappa_{r}}{\left\|\operatorname{grad} F_{b}\right\|}\left\{-\varepsilon^{2} \frac{\partial \theta^{+}}{\partial x} \frac{\partial b}{\partial x}-\varepsilon^{2} \frac{\partial \theta^{+}}{\partial y} \frac{\partial b}{\partial y}+\frac{\partial \theta^{+}}{\partial z}\right\} \\
& -\frac{\alpha_{t}}{\left\|\operatorname{grad} F_{b}\right\|}\left\{\left(v_{\mathrm{sl}}\right)_{x} \frac{\partial b}{\partial x}\left(-p+\varepsilon^{2} \sigma_{x}^{R}\right)+\varepsilon^{2}\left(v_{\mathrm{sl}}\right)_{x} \frac{\partial b}{\partial y} \sigma_{x y}-\left(v_{\mathrm{sl}}\right)_{x} \sigma_{x z}\right. \\
& +\varepsilon^{2}\left(v_{\mathrm{sl}}\right)_{y} \frac{\partial b}{\partial x} \sigma_{x y}+\left(v_{\mathrm{sl}}\right)_{y} \frac{\partial b}{\partial y}\left(-p+\varepsilon^{2} \sigma_{y}^{R}\right)-\left(v_{\mathrm{sl}}\right)_{y} \sigma_{y z}+\varepsilon^{2}\left(v_{\mathrm{sl}}\right)_{z} \frac{\partial b}{\partial x} \sigma_{x z} \\
& \left.+\varepsilon^{2}\left(v_{\mathrm{sl}}\right)_{z} \frac{\partial b}{\partial y} \sigma_{y z}-\left(v_{\mathrm{sl}}\right)_{z}\left(-p+\varepsilon^{2} \sigma_{z}^{R}\right)\right\},
\end{aligned}
$$


and application of the SIA gives

$$
\mathcal{P}_{b}^{w}=\frac{\mathcal{D} \alpha_{t}}{\alpha} \kappa \frac{\partial \theta}{\partial z}-\frac{\mathcal{D} \alpha_{t}}{\alpha} \frac{\left[\kappa_{r}\right]}{[\kappa]} \kappa_{r} \frac{\partial \theta^{+}}{\partial z}+\alpha_{t}\left(\left(v_{\mathrm{sl}}\right)_{x} \sigma_{x z}+\left(v_{\mathrm{sl}}\right)_{y} \sigma_{y z}\right) ;
$$

the pressure terms in (4.65) cancel due to (4.55) - (4.57).

Finally, there is continuity of the temperature (2.57),

$$
\theta=\theta^{+}=\theta_{M} .
$$

\subsubsection{Boundary conditions at the lithosphere base}

The expressions for grad $F_{r}$ and $\left\|\operatorname{grad} F_{r}\right\|$ have the same form as for the cold ice base (see (4.41) - (4.43)); replacing $b$ by $b_{r}$ and $F_{b}$ by $F_{r}$. Thus, with $\boldsymbol{n}=\operatorname{grad} F_{r} /\left\|\operatorname{grad} F_{r}\right\|$, from (2.58)

$$
\kappa_{r}\left(\varepsilon^{2} \frac{\partial b_{r}}{\partial x} \frac{\partial \theta^{-}}{\partial x}+\varepsilon^{2} \frac{\partial b_{r}}{\partial y} \frac{\partial \theta^{-}}{\partial y}-\frac{\partial \theta^{-}}{\partial z}\right)=\mathcal{N}_{r} Q_{\text {geoth }}^{\perp},
$$

and in the SIA limit,

$$
\kappa_{r} \frac{\partial \theta^{-}}{\partial z}=-\mathcal{N}_{r} Q_{\text {geoth }}^{\perp}
$$

\subsubsection{Transition conditions at the CTS}

As for the case of the free surface, the kinematic condition (2.60),

$$
\frac{\partial z_{m}}{\partial t}+v_{x} \frac{\partial z_{m}}{\partial x}+v_{y} \frac{\partial z_{m}}{\partial y}-v_{z}=\left(1+\varepsilon^{2}\left(\frac{\partial z_{m}}{\partial x}\right)^{2}+\varepsilon^{2}\left(\frac{\partial z_{m}}{\partial y}\right)^{2}\right)^{1 / 2} a_{m}^{\perp},
$$

reduces in the SIA limit to

$$
\frac{\partial z_{m}}{\partial t}+v_{x} \frac{\partial z_{m}}{\partial x}+v_{y} \frac{\partial z_{m}}{\partial y}-v_{z}=a_{m}^{\perp} .
$$

$\operatorname{grad} F_{m},\left\|\operatorname{grad} F_{m}\right\|$ and $\boldsymbol{n}=\operatorname{grad} F_{m} /\left\|\operatorname{grad} F_{m}\right\|$ are evaluated as before.

Continuity of temperature and velocity (eqs. (2.61) - (2.63)) require

$$
\theta^{+}=\theta^{-}=\theta_{M}
$$

and

$$
v_{x}^{+}=v_{x}^{-}, \quad v_{y}^{+}=v_{y}^{-}, \quad v_{z}^{+}=v_{z}^{-} .
$$

The momentum jump relation (2.64) in scaled form is

$$
\begin{gathered}
{\left[\left[-\left(-p+\varepsilon^{2} \sigma_{x}^{R}\right) \frac{\partial z_{m}}{\partial x}-\varepsilon^{2} \sigma_{x y} \frac{\partial z_{m}}{\partial y}+\sigma_{x z}\right]\right]=0,} \\
{\left[\left[-\varepsilon^{2} \sigma_{x y} \frac{\partial z_{m}}{\partial x}-\left(-p+\varepsilon^{2} \sigma_{y}^{R}\right) \frac{\partial z_{m}}{\partial y}+\sigma_{y z}\right]\right]=0,} \\
{\left[\left[-\varepsilon^{2} \sigma_{x z} \frac{\partial z_{m}}{\partial x}-\varepsilon^{2} \sigma_{y z} \frac{\partial z_{m}}{\partial y}+\left(-p+\varepsilon^{2} \sigma_{z}^{R}\right)\right]\right]=0,}
\end{gathered}
$$

and in the SIA limit,

$$
p^{+}=p^{-}, \quad \sigma_{x z}^{+}=\sigma_{x z}^{-}, \quad \sigma_{y z}^{+}=\sigma_{y z}^{-} .
$$

Finally, from the energy jump relation (2.70) and the thermal and water-content jump relations (2.73) $-(2.78)$, it is inferred that 
i) $-\left(w_{x}-v_{w, x}\right) \frac{\partial z_{m}}{\partial x}-\left(w_{y}-v_{w, y}\right) \frac{\partial z_{m}}{\partial y}+\left(w_{z}-v_{w, z}\right)>0$ (melting condition):

$$
\begin{gathered}
-\left(w_{x}-v_{w, x}\right) \frac{\partial z_{m}}{\partial x}-\left(w_{y}-v_{w, y}\right) \frac{\partial z_{m}}{\partial y}+\left(w_{z}-v_{w, z}\right)>0: \\
\omega^{-}=0 \\
-\varepsilon^{2} \frac{\partial \theta^{+}}{\partial x} \frac{\partial z_{m}}{\partial x}-\varepsilon^{2} \frac{\partial \theta^{+}}{\partial y} \frac{\partial z_{m}}{\partial y}+\frac{\partial \theta^{+}}{\partial z}=-\varepsilon^{2} \frac{\partial \theta_{M}^{-}}{\partial x} \frac{\partial z_{m}}{\partial x}-\varepsilon^{2} \frac{\partial \theta_{M}^{-}}{\partial y} \frac{\partial z_{m}}{\partial y}+\frac{\partial \theta_{M}^{-}}{\partial z}
\end{gathered}
$$

and the latter reduces in the SIA limit to

$$
\frac{\partial \theta^{+}}{\partial z}=\frac{\partial \theta_{M}^{-}}{\partial z}
$$

ii) $-\left(w_{x}-v_{w, x}\right) \frac{\partial z_{m}}{\partial x}-\left(w_{y}-v_{w, y}\right) \frac{\partial z_{m}}{\partial y}+\left(w_{z}-v_{w, z}\right)<0$ (freezing condition):

In this case, (2.70) is needed in its full form, which, with the diffusion law (2.19), becomes

$$
\begin{aligned}
& \frac{\mathcal{D} \kappa}{\left\|\operatorname{grad} F_{m}\right\|}\left(-\varepsilon^{2} \frac{\partial \theta^{+}}{\partial x} \frac{\partial z_{m}}{\partial x}-\varepsilon^{2} \frac{\partial \theta^{+}}{\partial y} \frac{\partial z_{m}}{\partial y}+\frac{\partial \theta^{+}}{\partial z}\right) \\
& -\frac{\mathcal{D} \kappa}{\left\|\operatorname{grad} F_{m}\right\|}\left(-\varepsilon^{2} \frac{\partial \theta_{M}^{-}}{\partial x} \frac{\partial z_{m}}{\partial x}-\varepsilon^{2} \frac{\partial \theta_{M}^{-}}{\partial y} \frac{\partial z_{m}}{\partial y}+\frac{\partial \theta_{M}^{-}}{\partial z}\right) \\
& -\frac{\alpha}{\alpha_{t}} \frac{\mathcal{D}_{t}}{\left\|\operatorname{grad} F_{m}\right\|}\left(-\varepsilon^{2} \frac{\partial \omega^{-}}{\partial x} \frac{\partial z_{m}}{\partial x}-\varepsilon^{2} \frac{\partial \omega^{-}}{\partial y} \frac{\partial z_{m}}{\partial y}+\frac{\partial \omega^{-}}{\partial z}\right)=\frac{\alpha}{\alpha_{t}} \omega^{-} a_{m}^{\perp},
\end{aligned}
$$

and in the SIA limit

$$
\mathcal{D} \kappa \frac{\partial \theta^{+}}{\partial z}-\mathcal{D} \kappa \frac{\partial \theta_{M}^{-}}{\partial z}-\frac{\alpha}{\alpha_{t}} \mathcal{D}_{t} \frac{\partial \omega^{-}}{\partial z}=\frac{\alpha}{\alpha_{t}} \omega^{-} a_{m}^{\perp}
$$

iii) $-\left(w_{x}-v_{w, x}\right) \frac{\partial z_{m}}{\partial x}-\left(w_{y}-v_{w, y}\right) \frac{\partial z_{m}}{\partial y}+\left(w_{z}-v_{w, z}\right)=0$ (parallel-flow condition):

$$
\omega^{-} \geq 0 \quad \text { (undetermined). }
$$

Moreover, continuity of the temperature gradient must hold analogous to case (i) above (eqs. (4.79) and $(4.80))$.

\subsection{Partial integration of the polythermal SIA equations}

The above derived model equations, subjected to the shallow-ice approximation (SIA), can be partly integrated analytically. It is now demonstrated that the dominant stresses $p, \sigma_{x z}$ and $\sigma_{y z}$ can be obtained analytically, and the calculation of the three components $v_{x}, v_{y}$ and $v_{z}$ of the velocity field reduces to simple numerical quadratures. The actual "hard" numerics are therefore restricted to the computation of the temperature and water-content fields, respectively, and the evolution of the free surface, the ice base and the CTS of the polythermal ice sheet.

\subsubsection{Calculation of the stresses}

Integrating eq. (4.11) subject to the boundary condition (4.37) and the transition condition (4.77) gives the simple pressure distribution

$$
p(x, y, z, t)=h(x, y, t)-z,
$$


which is a purely hydrostatic distribution. Using this result in eqs. (4.9), (4.10), and integrating subject to the boundary and transition conditions (4.38) and (4.77), yields

$$
\begin{aligned}
\sigma_{x z} & =-\frac{\partial h}{\partial x}(h-z), \\
\sigma_{y z} & =-\frac{\partial h}{\partial y}(h-z) .
\end{aligned}
$$

Thus, the shear stresses are a product of surface gradient and overburden (hydrostatic) pressure - a classical glaciological result. From (4.21), the effective shear stress becomes

$$
\sigma=(h-z) \sqrt{\left(\frac{\partial h}{\partial x}\right)^{2}+\left(\frac{\partial h}{\partial y}\right)^{2}} .
$$

Note that the above results are equally valid in the cold as well as in the temperate regions of the ice sheet.

The remaining, very small stresses $\sigma_{x}^{R}, \sigma_{y}^{R}, \sigma_{z}^{R}$ and $\sigma_{x y}$, are not given by the leading-order equations (but they could be calculated from the leading-order situation), nor are they required.

\subsubsection{Calculation of the velocity}

The horizontal velocities $v_{x}$ and $v_{y}$ are obtained by integrating the last two equations (4.18), (4.19) of the stress-strain-rate relation with $\sigma_{x z}$ and $\sigma_{y z}$ given by (4.85) and (4.86):

$$
\begin{aligned}
& v_{x}=v_{x, b}-2 \mathcal{K} \frac{\partial h}{\partial x} \int_{b}^{z} E A_{(t)}(\cdot) f_{(t)}(\sigma)\left(h-z^{\prime}\right) d z^{\prime}, \\
& v_{y}=v_{y, b}-2 \mathcal{K} \frac{\partial h}{\partial y} \int_{b}^{z} E A_{(t)}(\cdot) f_{(t)}(\sigma)\left(h-z^{\prime}\right) d z^{\prime},
\end{aligned}
$$

in which

$$
A_{(t)}(\cdot)=\left\{\begin{array}{lll}
A\left(\theta^{\prime}\right) & \text { for } z>z_{m} & \text { (cold regions) } \\
A_{t}(\omega) & \text { for } z<z_{m} & \text { (temperate regions) }
\end{array}\right.
$$

and

$$
f_{(t)}(\sigma)=\left\{\begin{array}{lll}
f(\sigma) & \text { for } z>z_{m} & \text { (cold regions), } \\
f_{t}(\sigma) & \text { for } z<z_{m} & \text { (temperate regions). }
\end{array}\right.
$$

The integration constants $v_{x, b}$ and $v_{y, b}$ represent the corresponding basal velocities, and are given by the sliding law (4.49), (4.50) with the above values for $\sigma_{x z}$ and $\sigma_{y z}$ :

$$
\begin{aligned}
v_{x, b}=\left(v_{\mathrm{sl}}\right)_{x} & =-\mathcal{F}_{(t)} C_{(t)} \frac{\partial h}{\partial x}(h-b), \\
v_{y, b}=\left(v_{\mathrm{sl}}\right)_{y} & =-\mathcal{F}_{(t)} C_{(t)} \frac{\partial h}{\partial y}(h-b) .
\end{aligned}
$$

Here use has been made of the fact that the $x$ - and $y$ components of the lithosphere velocity vanish (see (4.28)), so that, according to (4.55), (4.56), the components of the sliding velocity and the corresponding components of the ice-base velocity are identical. Furthermore,

$$
\mathcal{F}_{(t)} C_{(t)}= \begin{cases}\mathcal{F} C & \text { for cold base, } \\ \mathcal{F}_{t} C_{t} & \text { for temperate base. }\end{cases}
$$

With these results for the horizontal velocities, further integration of the mass balance (4.5) gives the vertical velocity,

$$
v_{z}=-\int_{b}^{z}\left(\frac{\partial v_{x}}{\partial x}+\frac{\partial v_{y}}{\partial y}\right) d z^{\prime}+v_{z, b}
$$


In view of the kinematic conditions (4.40) and (4.62), the basal vertical velocity $v_{z, b}$ can be expressed in terms of $v_{x, b}$ and $v_{y, b}$.

To sum up, the velocities are given by the following relations:

$$
\begin{aligned}
v_{x} & =-\mathcal{F}_{(t)} C_{(t)} \frac{\partial h}{\partial x}(h-b)-2 \mathcal{K} \frac{\partial h}{\partial x} \int_{b}^{z} E A_{(t)}(\cdot) f_{(t)}(\sigma)\left(h-z^{\prime}\right) d z^{\prime} \\
v_{y} & =-\mathcal{F}_{(t)} C_{(t)} \frac{\partial h}{\partial y}(h-b)-2 \mathcal{K} \frac{\partial h}{\partial y} \int_{b}^{z} E A_{(t)}(\cdot) f_{(t)}(\sigma)\left(h-z^{\prime}\right) d z^{\prime} \\
v_{z} & =-\int_{b}^{z}\left(\frac{\partial v_{x}}{\partial x}+\frac{\partial v_{y}}{\partial y}\right) d z^{\prime}+\frac{\partial b}{\partial t}+v_{x, b} \frac{\partial b}{\partial x}+v_{y, b} \frac{\partial b}{\partial y}-[\omega] \dot{m}_{b}^{w}
\end{aligned}
$$

If the temperature and water-content fields are known, all integrals can be easily computed numerically. For the special case of an ice fluidity independent of temperature and water content $\left(A_{(t)}=\right.$ const), the integrations can even be performed analytically (see also Hutter, 1983).

Finally, note that

$$
\left(v_{x}, v_{y}\right) \propto-(\partial h / \partial x, \partial h / \partial y)=-\operatorname{grad}_{(x, y)} h
$$

that is, the horizontal velocity throughout a vertical column of ice is in the direction of steepest surface descent (cf. Hutter, 1983). This result allows the concistency of the SIA to be checked by comparing the velocity direction in ice-sheet boreholes.

\subsubsection{Evolution of the free surface}

An equation for the evolution of the free surface is derived by combining the mass balance (4.5) with the kinematic conditions (4.30), (4.40), (4.62) for the free surface and the ice base. Integration of the mass balance (4.5) from the ice base to the free surface yields, with the Leibniz rule,

$$
\begin{aligned}
v_{z, s}-v_{z, b}= & -\frac{\partial}{\partial x} \int_{b}^{h} v_{x} d z^{\prime}+v_{x, s} \frac{\partial h}{\partial x}-v_{x, b} \frac{\partial b}{\partial x} \\
& -\frac{\partial}{\partial y} \int_{b}^{h} v_{y} d z^{\prime}+v_{y, s} \frac{\partial h}{\partial y}-v_{y, b} \frac{\partial b}{\partial y} .
\end{aligned}
$$

In view of the kinematic conditions (4.30), (4.40) and (4.62), this takes the form

$$
\frac{\partial H}{\partial t}=\frac{\partial(h-b)}{\partial t}=-\frac{\partial}{\partial x} \int_{b}^{h} v_{x} d z^{\prime}-\frac{\partial}{\partial y} \int_{b}^{h} v_{y} d z^{\prime}+a_{s}^{\perp}-[\omega] \dot{m}_{b}^{w},
$$

which balances the temporal change of ice thickness with the horizontal divergence of the vertically integrated horizontal velocity, the accumulation-ablation function (climatic boundary condition) and the water mass flux into the base.

\subsubsection{Evolution of the CTS}

In a similar way an evolution equation for the CTS can be obtained. Integrating the mass balance (4.5) from the ice base to the CTS,

$$
\begin{aligned}
v_{z, m}-v_{z, b}= & -\frac{\partial}{\partial x} \int_{b}^{z_{m}} v_{x} d z^{\prime}+v_{x, m} \frac{\partial z_{m}}{\partial x}-v_{x, b} \frac{\partial b}{\partial x} \\
& -\frac{\partial}{\partial y} \int_{b}^{z_{m}} v_{y} d z^{\prime}+v_{y, m} \frac{\partial z_{m}}{\partial y}-v_{y, b} \frac{\partial b}{\partial y}
\end{aligned}
$$

and with the kinematic conditions (4.62) for the ice base and (4.71) for the CTS, it follows that

$$
\frac{\partial\left(z_{m}-b\right)}{\partial t}=-\frac{\partial}{\partial x} \int_{b}^{z_{m}} v_{x} d z^{\prime}-\frac{\partial}{\partial y} \int_{b}^{z_{m}} v_{y} d z^{\prime}+a_{m}^{\perp}-[\omega] \dot{m}_{b}^{w}
$$


The interpretation of this result is basically the same as for the surface evolution equation, but here the ice volume flux through the CTS, $a_{m}^{\perp}$, is an unknown inner variable, not a boundary source term.

\subsubsection{Evolution of the lithosphere surface}

No further calculation is required here, as eq. (4.27) is taken over unchanged,

$$
\frac{\partial b}{\partial t}=-\frac{1}{\mathcal{T}_{r}}\left[b-\left(b_{0}-\frac{\rho}{\rho_{a}} H\right)\right] .
$$

\subsubsection{Temperature and water content}

As already noted, the equations of $\S 4.2$ for the temperature field and the water-content field must be solved numerically. The water-content equation (4.24) can be slightly simplified by substituting the result (4.84) obtained for the hydrostatic pressure field. The temperature evolution equation for the cold region (4.13) reads

$$
\frac{\partial \theta}{\partial t}+v_{x} \frac{\partial \theta}{\partial x}+v_{y} \frac{\partial \theta}{\partial y}+v_{z} \frac{\partial \theta}{\partial z}=\frac{\mathcal{D}}{c} \frac{\partial}{\partial z}\left(\kappa \frac{\partial \theta}{\partial z}\right)+2 \frac{\alpha}{c} \mathcal{K} E A\left(\theta^{\prime}\right) f(\sigma) \sigma^{2} .
$$

In the temperate regions, the temperature is uniquely determined by the pressure; thus, from eqs. (4.22) and (4.84)

$$
\theta=\theta_{M}=-\mathcal{B}(h(x, y, t)-z)
$$

and consequently,

$$
\frac{\partial \theta_{M}}{\partial t}+v_{x} \frac{\partial \theta_{M}}{\partial x}+v_{y} \frac{\partial \theta_{M}}{\partial y}+v_{z} \frac{\partial \theta_{M}}{\partial z}=-\mathcal{B}\left(\frac{\partial h}{\partial t}+v_{x} \frac{\partial h}{\partial x}+v_{y} \frac{\partial h}{\partial y}-v_{z}\right)
$$

and

$$
\frac{\partial}{\partial z}\left(\kappa \frac{\partial \theta_{M}}{\partial z}\right)=\mathcal{B} \frac{\partial \kappa}{\partial z}=\mathcal{B} \frac{\partial \kappa}{\partial \theta} \frac{\partial \theta}{\partial z}=\mathcal{B}^{2} \frac{\partial \kappa}{\partial \theta} .
$$

For the water-content-evolution equation in the temperate regions (4.24) it follows that

$$
\begin{aligned}
& \frac{\partial \omega}{\partial t}+v_{x} \frac{\partial \omega}{\partial x}+v_{y} \frac{\partial \omega}{\partial y}+v_{z} \frac{\partial \omega}{\partial z}=\mathcal{D}_{t} \frac{\partial^{2} \omega}{\partial z^{2}}+\frac{\mathcal{D} \alpha_{t}}{\alpha} \mathcal{B}^{2} \frac{\partial \kappa}{\partial \theta} \\
& +\frac{c \alpha_{t}}{\alpha} \mathcal{B}\left(\frac{\partial h}{\partial t}+v_{x} \frac{\partial h}{\partial x}+v_{y} \frac{\partial h}{\partial y}-v_{z}\right)+2 \alpha_{t} \mathcal{K} E A_{t}(\omega) f_{t}(\sigma) \sigma^{2}
\end{aligned}
$$

in which the effective shear stress $\sigma$ is given by (4.87).

With (4.28) the temperature evolution equation for the lithosphere (4.26) is

$$
\frac{\partial \theta}{\partial t}+\frac{\partial b}{\partial t} \frac{\partial \theta}{\partial z}=\frac{\mathcal{D}_{r} \kappa_{r}}{c_{r}} \frac{\partial^{2} \theta}{\partial z^{2}} .
$$

At the cold free surface, the temperature is prescribed (eq. (4.39), a Dirichlet-type boundary condition),

$$
\theta=\theta_{s}(x, y, t) .
$$

The case of a temperate free surface, essentially irrelevant for ice sheets, will not be considered. For a cold ice base, (4.59) and (4.60) hold,

$$
\begin{aligned}
\kappa \frac{\partial \theta}{\partial z}-\frac{\left[\kappa_{r}\right]}{[\kappa]} \kappa_{r} \frac{\partial \theta^{+}}{\partial z}= & -\frac{\alpha}{\mathcal{D}}\left[\left(v_{\mathrm{sl}}\right)_{x} \sigma_{x z}+\left(v_{\mathrm{sl}}\right)_{y} \sigma_{y z}\right], \\
\theta & =\theta^{+},
\end{aligned}
$$


and in case of a temperate ice base, (4.64), (4.66) and (4.67) apply,

$$
\begin{gathered}
\mathcal{D}_{t} \frac{\partial \omega}{\partial z}=(1-[\omega] \omega) \dot{m}_{b}^{w}-\mathcal{P}_{b}^{w}, \\
\theta=\theta^{+}=\theta_{M}, \\
\mathcal{P}_{b}^{w}=\frac{\mathcal{D} \alpha_{t}}{\alpha} \kappa \frac{\partial \theta}{\partial z}-\frac{\mathcal{D} \alpha_{t}}{\alpha} \frac{\left[\kappa_{r}\right]}{[\kappa]} \kappa_{r} \frac{\partial \theta^{+}}{\partial z}+\alpha_{t}\left(\left(v_{\mathrm{sl}}\right)_{x} \sigma_{x z}+\left(v_{\mathrm{sl}}\right)_{y} \sigma_{y z}\right) .
\end{gathered}
$$

Eq. (4.114) is required only if the temperate ice base is overlain by a temperate ice layer of non-vanishing thickness. In this case, (4.116) can further be simplified, because then $\partial \theta / \partial z=\mathcal{B}$ holds due to (4.106). In the case of a temperate ice base overlain by cold ice, however, this is not valid.

At the lithosphere base, (4.69) holds,

$$
\kappa_{r} \frac{\partial \theta^{-}}{\partial z}=-\mathcal{N}_{r} Q_{\text {geoth }}^{\perp}
$$

There remain the transition conditions at the CTS, (4.72), (4.78), (4.80) and (4.82):

$$
\theta^{+}=\theta^{-}=\theta_{M} .
$$

i) $-\left(w_{x}-v_{w, x}\right) \frac{\partial z_{m}}{\partial x}-\left(w_{y}-v_{w, y}\right) \frac{\partial z_{m}}{\partial y}+\left(w_{z}-v_{w, z}\right)>0$ (melting condition):

$$
\begin{aligned}
\omega^{-} & =0 \\
\frac{\partial \theta^{+}}{\partial z} & =\mathcal{B} .
\end{aligned}
$$

ii) $-\left(w_{x}-v_{w, x}\right) \frac{\partial z_{m}}{\partial x}-\left(w_{y}-v_{w, y}\right) \frac{\partial z_{m}}{\partial y}+\left(w_{z}-v_{w, z}\right)<0$ (freezing condition):

$$
\mathcal{D} \kappa\left(\frac{\partial \theta^{+}}{\partial z}-\mathcal{B}\right)-\frac{\alpha}{\alpha_{t}} \mathcal{D}_{t} \frac{\partial \omega^{-}}{\partial z}=\frac{\alpha}{\alpha_{t}} \omega^{-} a_{m}^{\perp}
$$

where $\partial \theta_{M}^{-} / \partial z=\mathcal{B}$ was used.

iii) $-\left(w_{x}-v_{w, x}\right) \frac{\partial z_{m}}{\partial x}-\left(w_{y}-v_{w, y}\right) \frac{\partial z_{m}}{\partial y}+\left(w_{z}-v_{w, z}\right)=0$ (parallel-flow condition):

$$
\begin{gathered}
\omega^{-} \geq 0 \quad \text { (undetermined) } \\
\frac{\partial \theta^{+}}{\partial z}=\mathcal{B} .
\end{gathered}
$$

Finally, some limitations of the shallow-ice approximation are noted. First, this limit excludes closed CTS lines with infinite gradient at a turning point, because owing to the requirement $\varepsilon \ll 1$ it only allows a CTS of small inclination. Inclusions of temperate ice in an environment of cold ice are therefore not accounted for, only temperate regions that reach the ice base, but the latter represents by far the most important case. Furthermore, the SIA entails singularities at ice margins and ice domes (maxima of the ice elevation above sea level) (see e.g. Hutter, 1983; Morland, 1984; Fowler, 1992; Hutter, 1993). Provided the basal sliding function $C_{(t)}$ is bounded, in the vicinity of an ice margin the ice thickness takes the form of a square root function with a vertical tangent at the margin, in contradiction to the shallowness assumption. At ice domes, use of a pseudoplastic power law creep response function $f_{(t)}(\sigma)=\sigma^{n-1}$ with $n>1$ (a conventional assumption, see $\S 5.1$ ) yields an infinite curvature of the ice surface, a consequence of the viscosity tending to infinity for small stresses and strain rates, respectively (Hutter et. al., 1986). These results have been derived for a purely cold-ice model, but they are applicable for polythermal ice sheets as well. Moreover, in the vicinity of ice domes the SIA assumption that the vertical velocity is of the order 
$\varepsilon \times$ horizontal velocity breaks down, because the velocity field is primarily vertical in that neighborhood; see eqs. (4.96), (4.97) und (4.98). Thus, the SIA is not uniformly valid, and matching procedures would be needed to connect inner and outer solutions. Such an extended asymptotic solution is, however, not required since the region around a dome is passive to leading order. In addition, it can be expected that a numerical solution of the model with discrete grid points smears out these local singularities, so that the remaining error is negligible.

\subsection{Compilation of the SIA equations in dimensional form}

For better clarity and interpretation, the partly integrated polythermal SIA equations derived in $\S 4.3$ are listed in dimensional form. They can be easily deduced from the dimensionless equations by setting all the typical values [.] to unity (see $\S 4.1$ ).

\section{Stresses:}

$$
\begin{aligned}
p & =\rho g(h-z) \\
\sigma_{x z} & =-\rho g(h-z) \frac{\partial h}{\partial x} \\
\sigma_{y z} & =-\rho g(h-z) \frac{\partial h}{\partial y} \\
\sigma & =\rho g(h-z) \sqrt{\left(\frac{\partial h}{\partial x}\right)^{2}+\left(\frac{\partial h}{\partial y}\right)^{2}} .
\end{aligned}
$$

Velocity:

$$
\begin{aligned}
v_{x} & =-\rho g(h-b) C_{(t)} \frac{\partial h}{\partial x}-2 \rho g \frac{\partial h}{\partial x} \int_{b}^{z} E A_{(t)}(\cdot) f_{(t)}(\sigma)\left(h-z^{\prime}\right) d z^{\prime} \\
v_{y} & =-\rho g(h-b) C_{(t)} \frac{\partial h}{\partial y}-2 \rho g \frac{\partial h}{\partial y} \int_{b}^{z} E A_{(t)}(\cdot) f_{(t)}(\sigma)\left(h-z^{\prime}\right) d z^{\prime} \\
v_{z} & =-\int_{b}^{z}\left(\frac{\partial v_{x}}{\partial x}+\frac{\partial v_{y}}{\partial y}\right) d z^{\prime}+\frac{\partial b}{\partial t}+v_{x, b} \frac{\partial b}{\partial x}+v_{y, b} \frac{\partial b}{\partial y}-\frac{\dot{m}_{b}^{w}}{\rho}
\end{aligned}
$$

Evolution of the free surface:

$$
\frac{\partial H}{\partial t}=\frac{\partial(h-b)}{\partial t}=-\frac{\partial Q_{x}}{\partial x}-\frac{\partial Q_{y}}{\partial y}+a_{s}^{\perp}-\frac{\dot{m}_{b}^{w}}{\rho},
$$

with the newly introduced mass flux $\boldsymbol{Q}$ defined as

$$
\left(Q_{x}, Q_{y}\right):=\int_{b}^{h}\left(v_{x}, v_{y}\right) d z^{\prime}
$$

Evolution of the CTS:

$$
\frac{\partial\left(z_{m}-b\right)}{\partial t}=-\frac{\partial}{\partial x} \int_{b}^{z_{m}} v_{x} d z^{\prime}-\frac{\partial}{\partial y} \int_{b}^{z_{m}} v_{y} d z^{\prime}+a_{m}^{\perp}-\frac{\dot{m}_{b}^{w}}{\rho} .
$$

Evolution of the ice base (lithosphere surface):

$$
\frac{\partial b}{\partial t}=-\frac{1}{\tau_{V}}\left[b-\left(b_{0}-\frac{\rho}{\rho_{a}} H\right)\right]
$$


Temperature and water content:

Temperature equation, cold regions:

$$
\frac{\partial T}{\partial t}+v_{x} \frac{\partial T}{\partial x}+v_{y} \frac{\partial T}{\partial y}+v_{z} \frac{\partial T}{\partial z}=\frac{1}{\rho c} \frac{\partial}{\partial z}\left(\kappa \frac{\partial T}{\partial z}\right)+\frac{2}{\rho c} E A\left(T^{\prime}\right) f(\sigma) \sigma^{2} .
$$

Temperature, temperate regions:

$$
T=T_{M}=T_{0}-\beta(h-z) .
$$

Water content equation, temperate regions:

$$
\begin{aligned}
& \frac{\partial \omega}{\partial t}+v_{x} \frac{\partial \omega}{\partial x}+v_{y} \frac{\partial \omega}{\partial y}+v_{z} \frac{\partial \omega}{\partial z}=\frac{\nu}{\rho} \frac{\partial^{2} \omega}{\partial z^{2}}+\frac{\beta^{2}}{\rho L} \frac{\partial \kappa}{\partial T} \\
& +\frac{c \beta}{L}\left(\frac{\partial h}{\partial t}+v_{x} \frac{\partial h}{\partial x}+v_{y} \frac{\partial h}{\partial y}-v_{z}\right)+\frac{2}{\rho L} E A_{t}(\omega) f_{t}(\sigma) \sigma^{2}-\frac{1}{\rho} D(\omega) .
\end{aligned}
$$

Here an additional term $-D(\omega) / \rho$ has been introduced; $D(\omega)$ is the water drainage function, corresponding to a negative volumetric water production; it represents a simple ad-hoc approach to describe the water drainage from the temperate-ice regions into the base. This is necessary because, as analytic estimates and preliminary numerical simulations without this term have shown, otherwise unrealistic and even nonphysical $(\omega>100 \%)$ values for the water content may result. Of course, this extraction process of water from the interior of the ice violates the local mass balance. More realistic drainage mechanisms, based on the combined effects of gravitation and interaction forces between ice and water, would require an even more complicated model with two separate momentum balances for ice and water, which is not pursued here (cf. Wu, 1996; Bauer, 1997).

Temperature equation, lithosphere:

$$
\frac{\partial T}{\partial t}+\frac{\partial b}{\partial t} \frac{\partial T}{\partial z}=\frac{\kappa_{r}}{\rho_{r} c_{r}} \frac{\partial^{2} T}{\partial z^{2}}
$$

Cold free surface:

$$
T=T_{s}(x, y, t)
$$

Cold ice base:

$$
\begin{aligned}
\kappa \frac{\partial T}{\partial z}-\kappa_{r} \frac{\partial T^{+}}{\partial z} & =-\left(v_{\mathrm{sl}}\right)_{x} \sigma_{x z}-\left(v_{\mathrm{sl}}\right)_{y} \sigma_{y z} \\
T & =T^{+} .
\end{aligned}
$$

Temperate ice base (not overlain by a temperate ice layer):

$$
\begin{aligned}
T & =T^{+}=T_{M}, \\
\mathcal{P}_{b}^{w} & =\frac{1}{L}\left\{\kappa \frac{\partial T}{\partial z}-\kappa_{r} \frac{\partial T^{+}}{\partial z}+\left(\left(v_{\mathrm{sl}}\right)_{x} \sigma_{x z}+\left(v_{\mathrm{sl}}\right)_{y} \sigma_{y z}\right)\right\} .
\end{aligned}
$$

Temperate ice base (overlain by a temperate ice layer):

$$
\begin{aligned}
\nu \frac{\partial \omega}{\partial z} & =(1-\omega) \dot{m}_{b}^{w}-\mathcal{P}_{b}^{w} \\
T & =T^{+}=T_{M} \\
\mathcal{P}_{b}^{w} & =\frac{1}{L}\left\{\kappa \beta-\kappa_{r} \frac{\partial T^{+}}{\partial z}+\left(\left(v_{\mathrm{sl}}\right)_{x} \sigma_{x z}+\left(v_{\mathrm{sl}}\right)_{y} \sigma_{y z}\right)\right\}
\end{aligned}
$$

Recall that in case of a negligible water diffusivity $\nu$, the water mass flux into the base, $\dot{m}_{b}^{w}$, that must be prescribed in general, can be expressed through (2.48) by the basal melting rate $\mathcal{P}_{b}^{w}$. Since the water content of temperate ice is assumed to be small (see also (5.7)), $\dot{m}_{b}^{w} \approx \mathcal{P}_{b}^{w}$ holds. 
Lithosphere base:

$$
\kappa_{r} \frac{\partial T^{-}}{\partial z}=-Q_{\text {geoth }}^{\perp}
$$

Transition conditions, CTS:

$$
T^{+}=T^{-}=T_{M}
$$

i) $-\left(w_{x}-v_{w, x}\right) \frac{\partial z_{m}}{\partial x}-\left(w_{y}-v_{w, y}\right) \frac{\partial z_{m}}{\partial y}+\left(w_{z}-v_{w, z}\right)>0$ (melting condition):

$$
\begin{gathered}
\omega^{-}=0 \\
\frac{\partial T^{+}}{\partial z}=\beta .
\end{gathered}
$$

ii) $-\left(w_{x}-v_{w, x}\right) \frac{\partial z_{m}}{\partial x}-\left(w_{y}-v_{w, y}\right) \frac{\partial z_{m}}{\partial y}+\left(w_{z}-v_{w, z}\right)<0$ (freezing condition):

$$
\kappa\left(\frac{\partial T^{+}}{\partial z}-\beta\right)-L \nu \frac{\partial \omega^{-}}{\partial z}=L \rho \omega^{-} a_{m}^{\perp}
$$

iii) $-\left(w_{x}-v_{w, x}\right) \frac{\partial z_{m}}{\partial x}-\left(w_{y}-v_{w, y}\right) \frac{\partial z_{m}}{\partial y}+\left(w_{z}-v_{w, z}\right)=0$ (parallel-flow condition):

$$
\begin{gathered}
\omega^{-} \geq 0 \quad(\text { undetermined }), \\
\frac{\partial T^{+}}{\partial z}=\beta .
\end{gathered}
$$

In contrast to these general distinguishing criteria, the distinctions for negligible water diffusion are simply determined by the sign of the ice volume flux through the CTS, $a_{m}^{\perp}$ (see $\S 2.2 .5$ ), thus $a_{m}^{\perp}>0$ for the melting condition (case i), $a_{m}^{\perp}<0$ for the freezing condition (case ii) and $a_{m}^{\perp}=0$ for the parallel-flow condition (case iii).

\section{A simulation of the Greenland Ice Sheet}

\subsection{Specification of physical quantities}

The polythermal SIA equations compiled in $\S 4.4$ contain a number of physical quantities that are still undefined. They are now specified.

Creep response function, cold and temperate ice:

Glen's flow law (cf. Glen, 1955; Nye, 1957; Hooke, 1981; Paterson, 1994)

$$
f(\sigma)=f_{t}(\sigma)=\sigma^{n-1} \quad \text { with } \quad n=3 .
$$

Rate factor, cold ice:

Arrhenius law for $T^{\prime} \leq-10^{\circ} \mathrm{C}$, Arrhenius-type fit for $-10^{\circ} \mathrm{C} \leq T^{\prime}<0^{\circ} \mathrm{C}$ (Paterson, 1994):

$$
A\left(T^{\prime}\right)= \begin{cases}A_{0} e^{-Q / R\left(T_{0}+T^{\prime}\right)} & \text { for } T^{\prime} \leq-10^{\circ} \mathrm{C}, \\ A_{0}^{\star} e^{-Q^{\star} / R\left(T_{0}+T^{\prime}\right)} & \text { for }-10^{\circ} \mathrm{C} \leq T^{\prime}<0^{\circ} \mathrm{C},\end{cases}
$$

with the activation energy $Q=60 \mathrm{~kJ} \mathrm{~mol}^{-1}$, the parameter $Q^{\star}=139 \mathrm{~kJ} \mathrm{~mol}^{-1}$, the universal gas constant $R$, and $A\left(T^{\prime}=-10^{\circ} \mathrm{C}\right)=5.2 \cdot 10^{-25} \mathrm{~s}^{-1} \mathrm{~Pa}^{-3}$ as a connecting value for the two temperature regimes, which determines $A_{0}$ and $A_{0}^{\star}$. 
Rate factor, temperate ice: Following Lliboutry \& Duval (1985),

$$
A_{t}(\omega)=A\left(T^{\prime}=0^{\circ} \mathrm{C}\right)(1+184 \omega) .
$$

Sliding law, cold base: Adhesion condition

$$
\boldsymbol{v}_{\mathrm{sl}}=\boldsymbol{v}_{b}=\mathbf{0}
$$

Sliding law, temperate base: Weertman-type sliding law

$$
\boldsymbol{v}_{\mathrm{sl}}=\boldsymbol{v}_{b}=-\frac{C_{\mathrm{sl}}}{\rho g} \frac{\left\|\boldsymbol{t}_{\|}\right\|^{p}}{(\rho g H)^{q}} \frac{\boldsymbol{t}_{\|}}{\left\|\boldsymbol{t}_{\|}\right\|} \quad \text { with } \quad p=3, q=2,
$$

from which

$$
\boldsymbol{v}_{\mathrm{sl}}=\boldsymbol{v}_{b}=-C_{\mathrm{sl}} H\|\operatorname{grad} h\|^{2} \operatorname{grad} h
$$

is obtained, with a sliding coefficient $C_{\mathrm{sl}}=6 \cdot 10^{4} \mathrm{a}^{-1}$ (Calov, 1994).

Water drainage function:

This is very problematic because of the lack of appropriate measurements. It is expected that the temperate ice can keep a certain amount of water, whereas additional water runs off downward through cracks, crevasses and grain boundaries. The assumption here is that up to a threshold value $\omega_{\max }$, drainage is negligible, and that any water surplus exceeding this threshold value is instantaneously drained, thus

$$
D(\omega)=\left\{\begin{array}{ll}
0 & \omega \leq \omega_{\max } \\
\infty & \omega>\omega_{\max }
\end{array}\right\} \quad\left(\text { with } \omega_{\max }=1 \%\right) .
$$

In view of sparseness of data on the water content of temperate glacier ice, this very simple parameterization has the advantage that only one unknown parameter $\omega_{\max }$ appears. The choice of $\omega_{\max }$ in (5.7) corresponds to the experimentally checked range of validity of the rate factor for temperate ice (5.3), cf. Lliboutry \& Duval (1985).

Further quantities are compiled in table 1.

\begin{tabular}{ll}
\hline quantity & value \\
\hline $\begin{array}{l}\text { density of ice, } \rho \\
\text { heat conductivity of ice, } \kappa \\
\text { specific heat of ice, } c\end{array}$ & $910 \mathrm{~kg} \mathrm{~m}^{-3}$ \\
latent heat of ice, $L$ & $9.828 e^{-0.0057 T[\mathrm{~K}]} \mathrm{W} \mathrm{m}^{-1} \mathrm{~K}^{-1}$ \\
Clausius-Clapeyron gradient, $\beta$ & $(146.3+7.253 T[\mathrm{~K}]) \mathrm{J} \mathrm{kg}^{-1} \mathrm{~K}^{-1}$ \\
water diffusivity, $\nu$ & $335 \mathrm{~kJ} \mathrm{~kg}^{-1}$ \\
density $\times$ specific heat of the & $8.7 \cdot 10^{-4} \mathrm{~K} \mathrm{~m}^{-1}$ \\
lithosphere, $\rho_{r} c_{r}$ & 0 \\
heat conductivity of the lithosphere, $\kappa_{r}$ & $3000 \mathrm{WJ} \mathrm{m}^{-1} \mathrm{~K}^{-1} \mathrm{~K}^{-1}$ \\
thickness of the upper lithosphere layer & \\
regarded by the model, $H_{r}$ & $5 \mathrm{~km}$ \\
time lag for bed adjustment, $\tau_{V}$ & $3000 \mathrm{a}$ \\
density of the asthenosphere, $\rho_{a}$ & $3300 \mathrm{~kg} \mathrm{~m}^{-3}$ \\
gravity acceleration, $g$ & $9.81 \mathrm{~m} \mathrm{~s}^{-2}$ \\
universal gas constant, $R$ & $8.314 \mathrm{~J} \mathrm{~mol}^{-1} \mathrm{~K}^{-1}$ \\
\hline
\end{tabular}

Table 1: Compilation of the physical parameters used in the model. References: $\rho, \beta$ : Calov $(1994) ; \tau_{V}$, $\rho_{a}$ : Abe-Ouchi (1993); $\kappa, c, \rho_{r} c_{r}, \kappa_{r}$ : Ritz (1987); L: Blatter (1991). 


\subsection{Evolution to the present-time steady state}

As an example of the application of the polythermal SIA equations to a real problem, a steady-state simulation under present climate conditions for the Greenland Ice Sheet is presented, based on the finitedifference program SICOPOLIS (SImulation COde for POLythermal Ice Sheets) using a $\sigma$-transformation in the vertical (i.e., vertical columns in the cold-ice domain, the temperate-ice domain and the lithosphere are mapped onto $[0,1]$ intervals) (Greve, 1995). A horizontal resolution of $40 \mathrm{~km}$ is applied, the vertical resolution is 51 grid points in the cold-ice domain, and 11 grid points in the temperate-ice domain and in the lithosphere. The time steps for eqs. (4.131), (4.134) are 10 years, and for eqs. (4.135), (4.137) are 100 years. Computation of the lithosphere temperature governed by eq. (4.138) has been switched off (instead, the geothermal heat flow has been imposed directly at the ice base), because the thermal inertia of the lithosphere is only important during the evolution and does not influence the final steady state. Since this is simply a demonstration of the applicability of the model, the numerical solution technique of SICOPOLIS is not discussed, for further information see Greve (1995).

The demonstration is a steady-state configuration under present climate conditions, described by the present distribution of the mean annual air temperature $T_{\mathrm{ma}}$ (assumed equal to the 10-m-firn temperature $T_{s}$ ) and the snowfall rate $S_{s}$ as known from measurements (Ohmura, 1987; Ohmura and Reeh, 1991), and a degree-day parameterization for the surface melting rate $M_{s}$ (Calov, 1994). For the geothermal heat flux, the standard value $Q_{\text {geoth }}^{\perp}=42 \mathrm{~mW} \mathrm{~m}^{-2}$ for precambrian shields is applied (Lee, 1970). The model time covers $t=0 \ldots 100000$ years, being sufficient to reach stationary conditions. As initial conditions for the topography the present values $b^{\text {today }}$ and $h^{\text {today }}$ (data from Letréguilly et. al., 1991a) are used, the initial temperature is uniformly set to $-10^{\circ} \mathrm{C}$, and for the initial age of the ice (see discussion below) the value 15000 years is applied.

The creep enhancement factor $E$ (see eqs. (2.4), (2.17)) is to take into account that glacial ice is less viscous than interglacial ice; however, the exact cause for this distinction is still unclear (possibly differences in dust content and/or induced anisotropies contribute to this phenomenon, cf. Paterson (1991), Paterson (1994), Svendsen and Hutter $(1996,1997))$. Here, the enhancement factor $E$ is coupled to the age of the ice $A$; the transition between Wisconsin ice age and Holocene interglacial is set at 11000 years before present:

$$
\begin{array}{ll}
E=1 & \text { if } A<11000 \text { years, } \\
E=3 & \text { if } A \geq 11000 \text { years. }
\end{array}
$$

Possible influences of Eemian or even older ice are not considered here, because this ice can only exist in very thin layers near the ice base where reliable calculation of the age is not certain (due to the nesessity of introducing a certain amount of numerical diffusion when solving the purely advective age equation $d A / d t=1)$.

The results of the simulation are depicted in figs. 10-16. When comparing the present surface topography from data (fig. 9) with the modelled topography (fig. 10), there is in general good agreement both for the surface topography itself and for the ice margin; the basic features are reproduced by the simulation. The north dome is $124 \mathrm{~m}$ too high $(3371 \mathrm{~m}$ instead of $3247 \mathrm{~m})$, the south dome $51 \mathrm{~m}$ too high $(2960 \mathrm{~m}$ intead of $2909 \mathrm{~m}$ ), and their positions are slightly shifted when compared with those of the measurements. Furthermore, the simulated ice margin tends to be more advanced toward the coast; in particular close to the north and east coast small ice tongues into actually ice-free land are produced. Therefore, the simulated ice-covered area $A_{i, b}=1.725 \cdot 10^{6} \mathrm{~km}^{2}$ is slightly bigger than the real value $1.682 \cdot 10^{6} \mathrm{~km}^{2}$.

Figs. 11, 12 show the mass flux $\boldsymbol{Q}$ and the ice surface velocity $\boldsymbol{v}_{s}$. The mass flux follows the direction of steepest surface slope, a consequence of the shallow ice approximation. Moreover, the flux away from the north dome takes place in pronounced drainage areas, separated by regions with distinctly reduced ice flow. Contrary to this, the drainage of the south dome appears to be more regular. This is caused by the distribution of the basal temperate ice regions (fig. 13), on which the ice is allowed to slide. They surround the south dome as an almost continuous band, whereas north of the Arctic circle essentially four temperate patches exist that form the drainage regions. This behaviour is an impressive demonstration of the great significance of temperate ice for the dynamic behaviour of ice sheets.

It is further noticeable that of all basal grid points overlain by a temperate ice layer of non-zero thickness, only one shows a CTS with freezing conditions (fig. 13). This is connected with the fact that, typically, the thickness of the basal temperate ice layer gradually increases from the inner ice sheet region downstream (toward the margin), and then decreases sharply in the immediate vicinity of the margin. 
This can be clearly seen for West Greenland in figs. 14, 15, showing west-east transects for the ice sheet at $y=-2280 \mathrm{~km}$ and $y=-1840 \mathrm{~km}$, respectively. Due to the steep decrease in thickness, freezing conditions at the CTS, expected close to the margin, cannot be resolved in general. Incidentally, the simulation results of a relatively thick temperate ice layer and high ice velocities in West Greenland in the transect at $y=-2280 \mathrm{~km}$ coincide with the presence of a very fast ice stream in this area ("Jacobshavns Isbræ"), for which Funk et. al. (1994) similarly obtain a temperate ice layer by applying a polythermal 2-d streamline model.

Finally, fig. 16 depicts the applied forcing $\Delta T_{\mathrm{ma}}(t) \equiv 0$, and the time evolutions for the maximum ice elevation above sea level $h_{\max }$ (taken at the north dome), the total ice volume $V_{\text {tot }}$, the maximum ice thickness $H_{\max }$, the temperate ice volume $V_{\text {temp }}$, the maximum thickness of the temperate ice layer $H_{t, \max }$, the ice covered basal area $A_{i, b}$ as well as the basal area covered by temperate ice $A_{t, b}$. A striking feature is the occurence of strong peaks for the quantities connected with temperate ice in the first 10000 model years, $V_{\text {temp }}, H_{t, \max }$ and $A_{t, b}$, coinciding with troughs of the topography quantities $h_{\max }, V_{\text {tot }}, H_{\max }$. This behaviour is apparently an effect of the arbitrarily chosen initial conditions (present topography, isothermal temperature conditions) and thus does not have any counterpart in the actual history of the Greenland Ice Sheet.

A variety of further simulations on steady-state as well as transient problems is described by Greve (1995, 1997), Greve and Hutter (1995), Greve and MacAyeal (1996), Hansen and Greve (1996), Hansen et. al. (1996) and Greve et. al. (1997). 


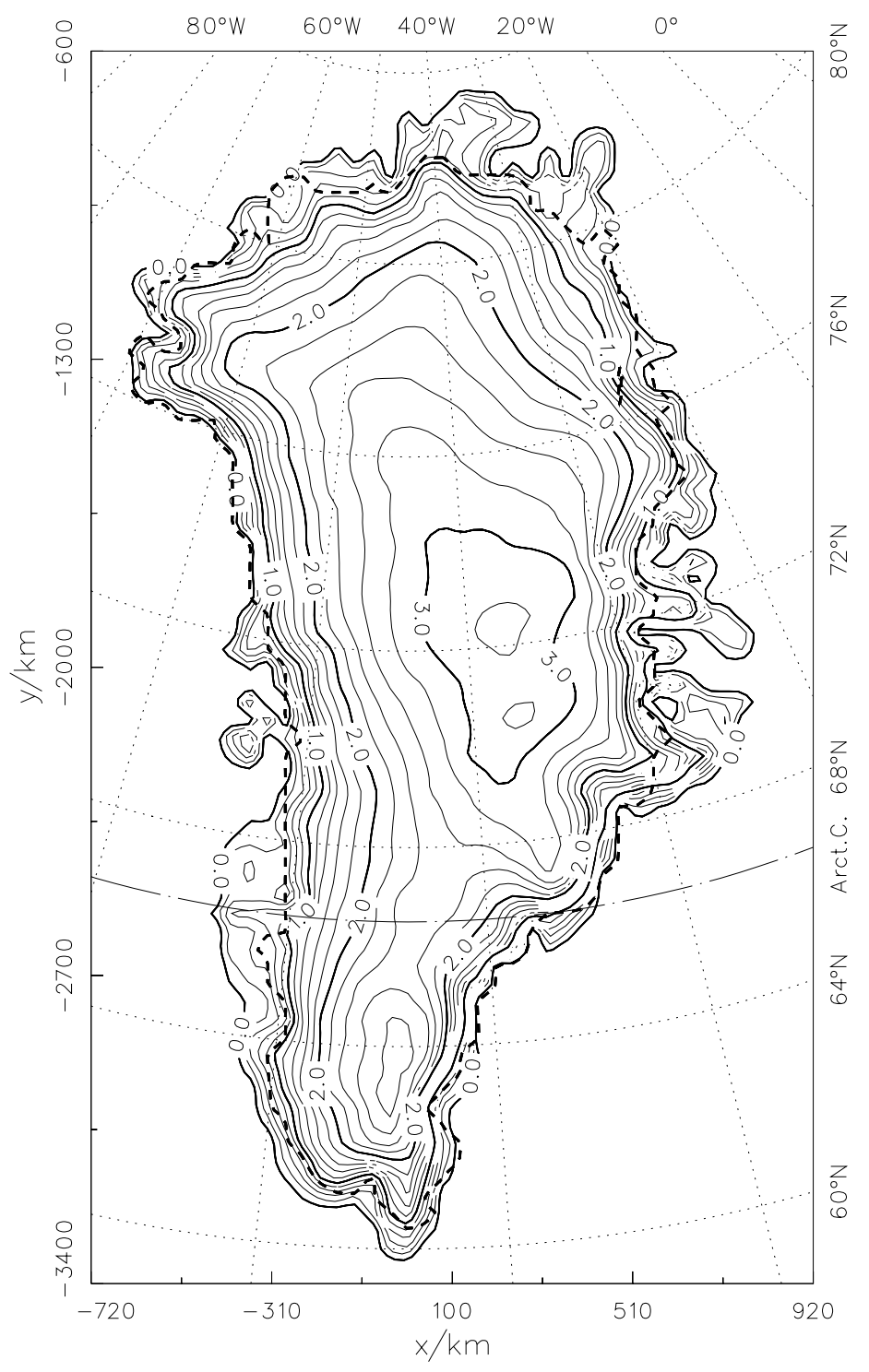

Figure 9: Measured surface topography $h$ of the present Greenland Ice Sheet (Letréguilly et. al., 1991a; in $\mathrm{km}$ above sea level). The spacing between the isolines is $200 \mathrm{~m}$. The dashed heavy line indicates the ice margin. 


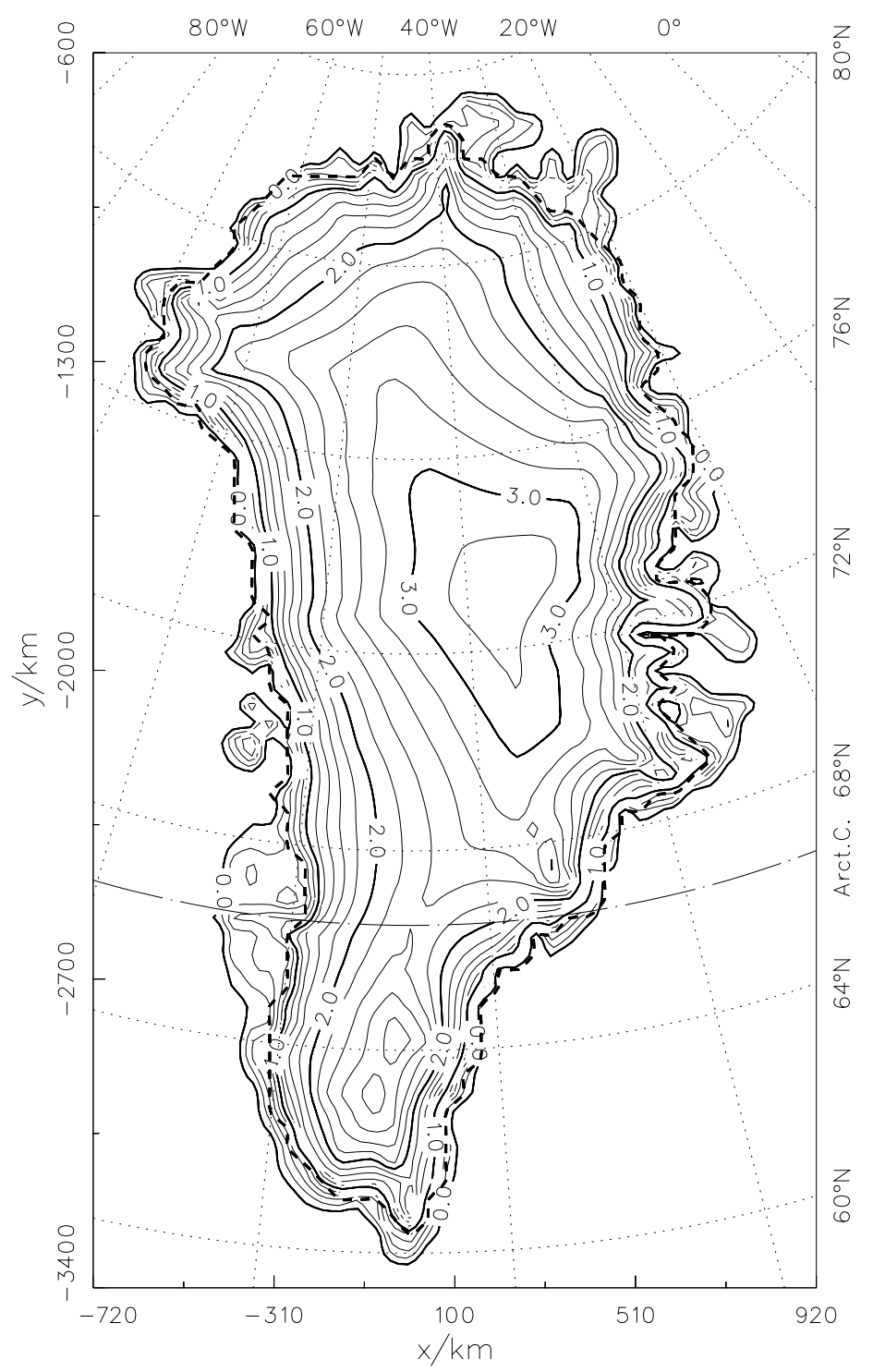

Figure 10: Final state of simulation: Topography of the ice surface (in $\mathrm{km}$ above sea level). The spacing between the isolines is $200 \mathrm{~m}$. The dashed heavy line indicates the ice margin. 


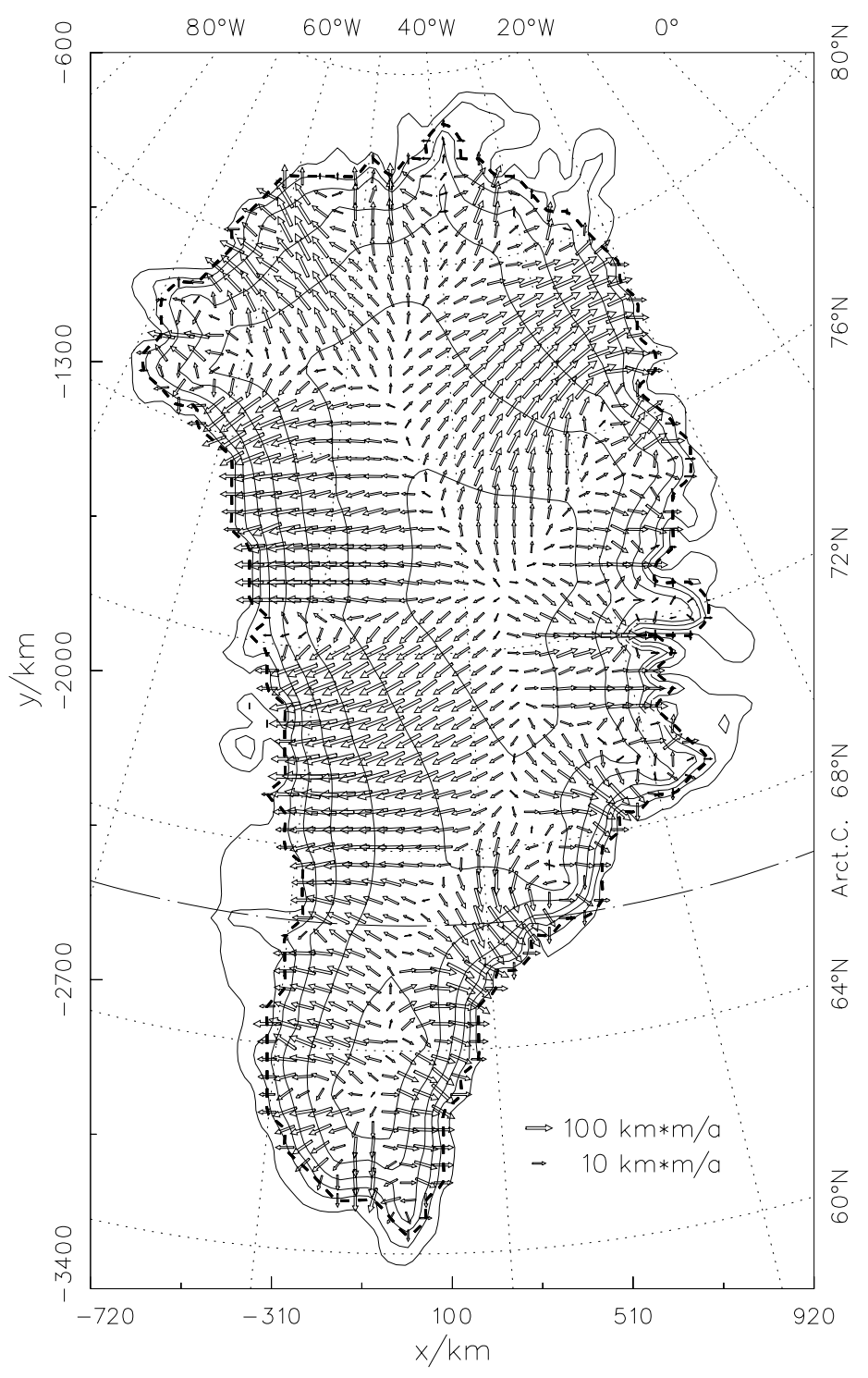

Figure 11: Final state of simulation: Horizontal mass flux. Double arrow length corresponds to tenfold mass flux. The dashed heavy line indicates the ice margin, the isolines the surface topography in $500 \mathrm{~m}$ intervals. 


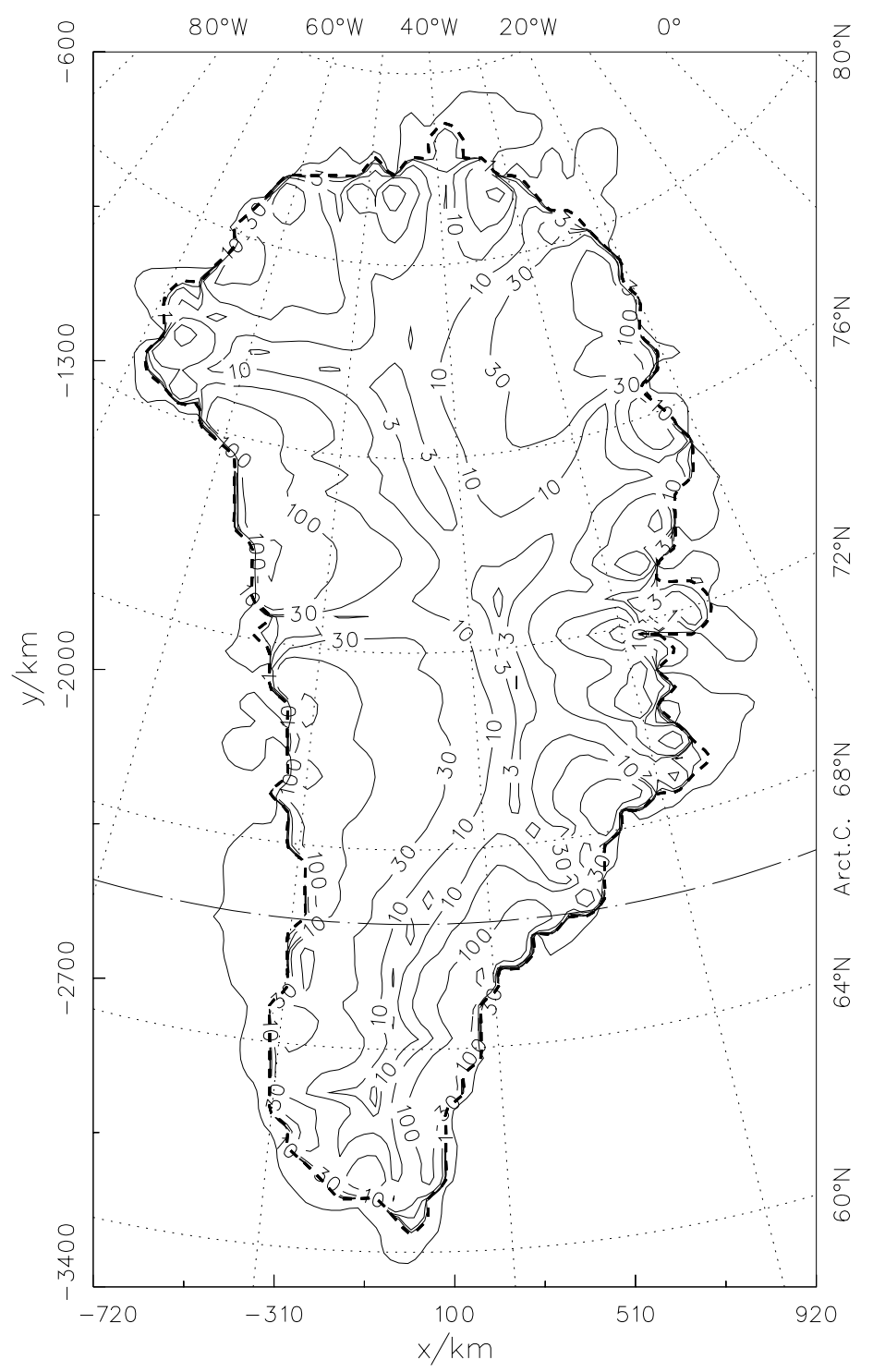

Figure 12: Final state of simulation: Surface velocity (in $\mathrm{km} / \mathrm{a}$ ). The isolines correspond to the values 1, $3,10,30 \ldots \mathrm{km} / \mathrm{a}$. The dashed heavy line indicates the ice margin. 


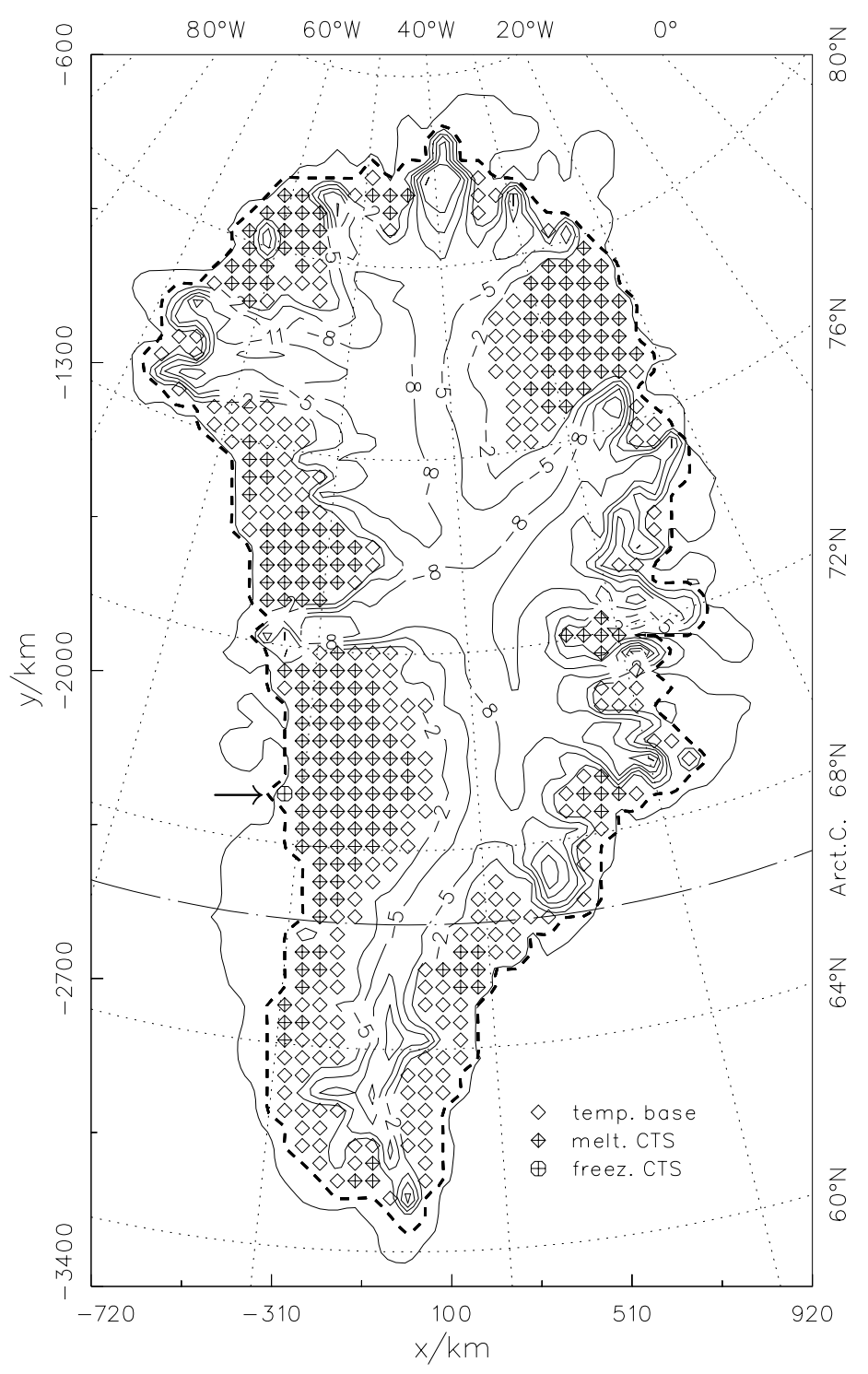

Figure 13: Final state of simulation: Homologous temperature at the ice base $\left(\right.$ in $\left.{ }^{\circ} \mathrm{C}\right)$. The spacing between the isolines is $3^{\circ} \mathrm{C}$. Open diamond symbols indicate positions where the basal ice is at the pressure melting point, yet with no temperate layer above, full diamonds (full circles, see $\rightarrow$ in the plot) indicate positions where there is a basal layer of temperate ice with a melting (freezing) CTS. The dashed heavy line indicates the ice margin. 

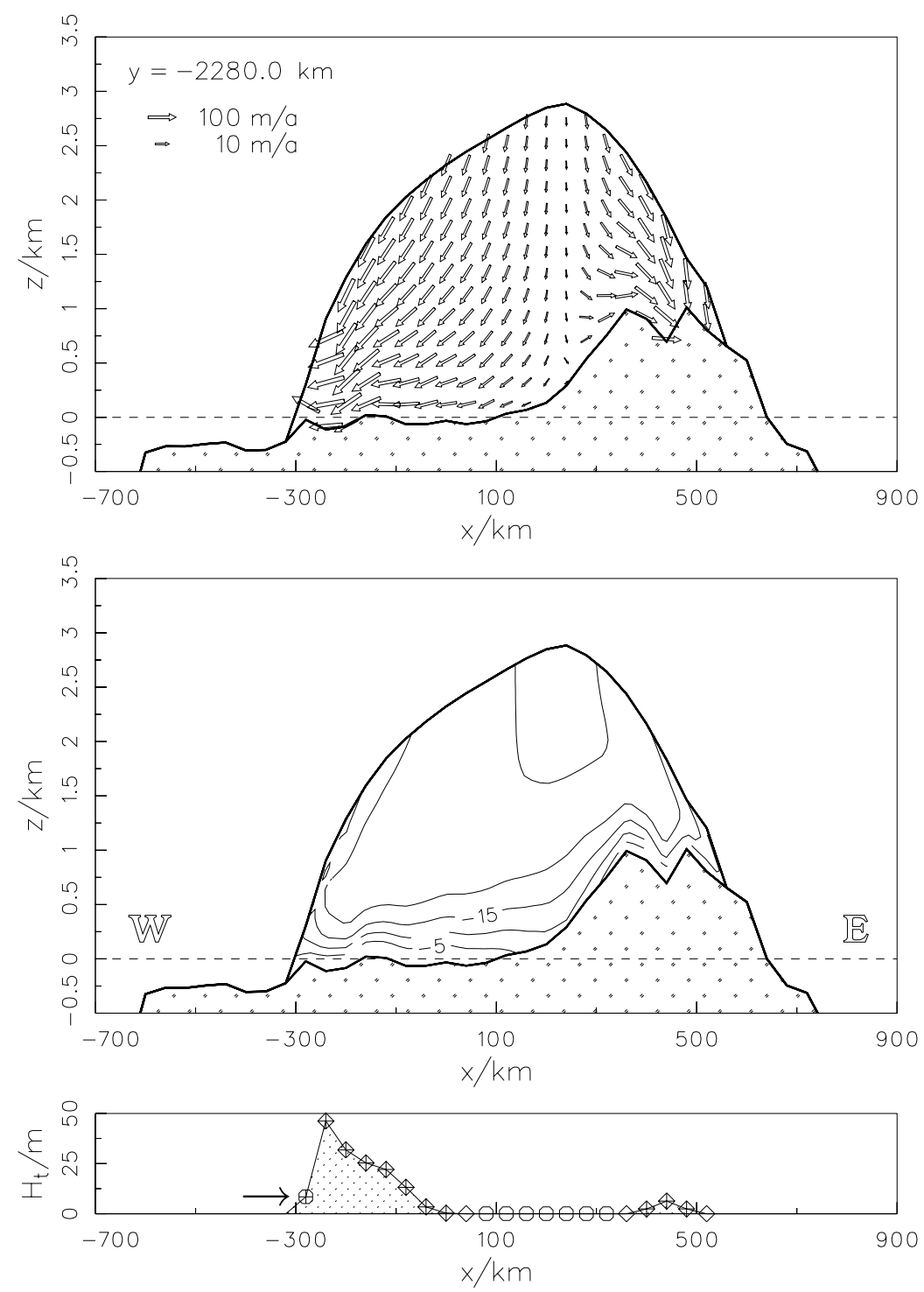

Figure 14: Final state of simulation: Transect at $y=-2280 \mathrm{~km}$. Top: ice velocity. Middle: homologous ice temperature $\left(\right.$ in ${ }^{\circ} \mathrm{C}$ ). Bottom: thickness of the basal layer of temperate ice (open circles: cold ice base; open diamonds: temperate ice base with no temperate ice layer above; full diamonds: basal layer of temperate ice with a melting CTS; full circle (see $\rightarrow$ in the plot): basal layer of temperate ice with a freezing CTS). 

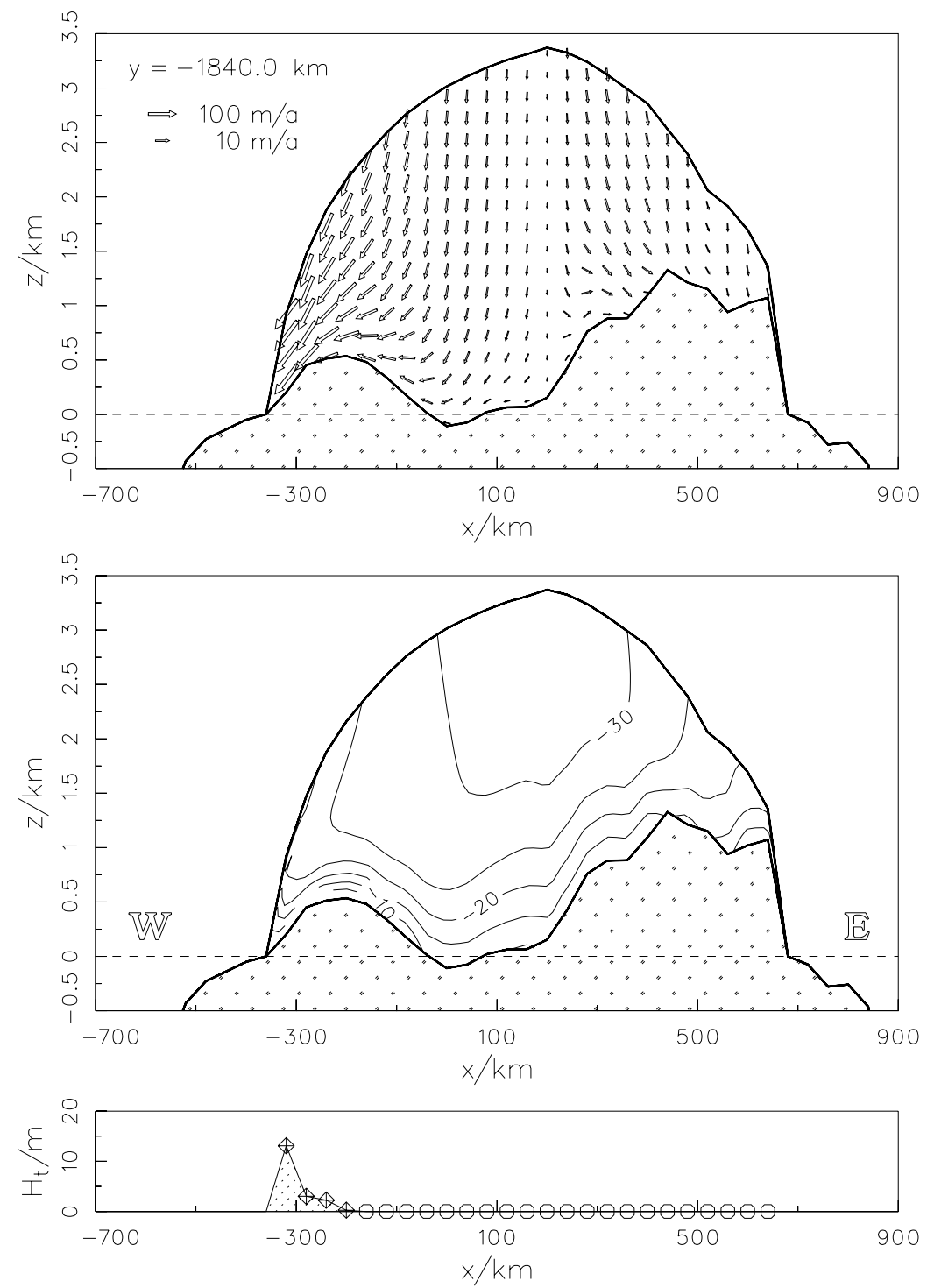

Figure 15: Final state of simulation: Transect at $y=-1840 \mathrm{~km}$. Top: ice velocity. Middle: homologous ice temperature (in ${ }^{\circ} \mathrm{C}$ ). Bottom: thickness of the basal layer of temperate ice (open circles: cold ice base; full diamonds: basal layer of temperate ice with a melting CTS). 

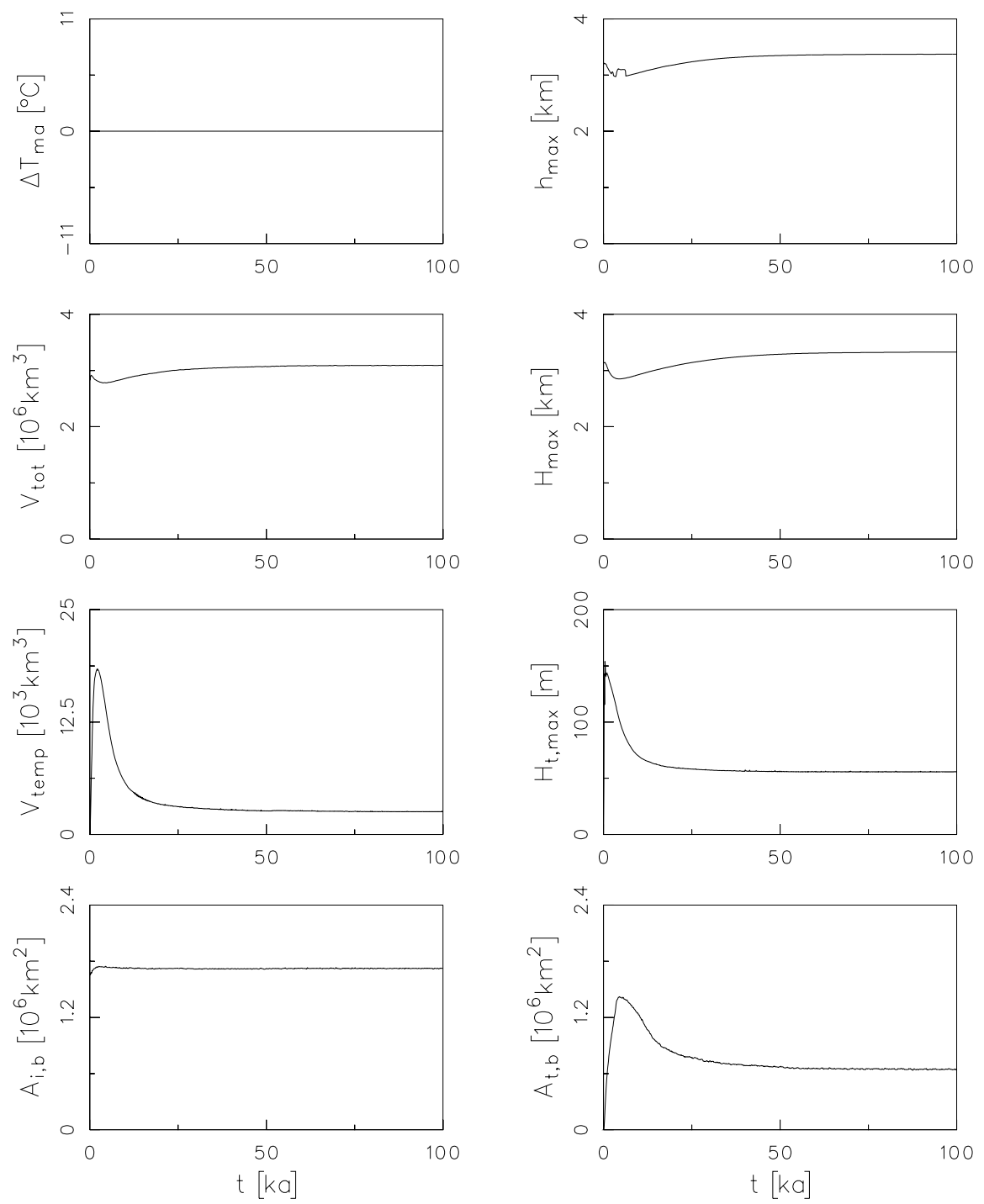

Figure 16: Simulation: Time evolution of $\Delta T_{\operatorname{ma}}, h_{\max }, V_{\text {tot }}, H_{\max }, V_{\text {temp }}, H_{t, \max }, A_{i, b}$ und $A_{t, b}$. The meaning of these quantities is explained in the main text. 


\section{Acknowledgements}

The author thanks Prof. Kolumban Hutter for his instructions when conducting this work, and for carefully reading and correcting the manuscript; Prof. Leslie Morland for his exceptionally thorough review, which lead to a considerable improvement of this paper in terms of clarity and language quality; Dr. Heinz Blatter for reviewing an earlier draft of the paper; Mr. Georg Bauer, Dr. Stefan Diebels and Mr. Magnus Weis for their time-consuming efforts to keep the Institute's workstations operating; Dr. Reinhard Calov for his aid in developing the numerical solution technique for the model equations; Dr. Anne Letréguilly for provision of the surface-topography and bedrock-topography data of Greenland. This work is part of the author's Ph.D. thesis (Greve, 1995). It was funded by a grant of the Studienstiftung des Deutschen Volkes.

\section{A Notation}

\begin{tabular}{|c|c|}
\hline$A$ & age of the ice \\
\hline$A_{i, b}$ & total ice-covered area \\
\hline$A_{t, b}$ & basal area covered by temperate ice \\
\hline$A\left(T^{\prime}\right)$ & rate factor of cold ice \\
\hline$A_{t}(\omega)$ & rate factor of temperate ice \\
\hline$A_{(t)}(\cdot)$ & rate factor of cold or temperate ice \\
\hline$a_{m}^{\perp}$ & volume flux through the CTS \\
\hline$a_{s}^{\perp}$ & accumulation-ablation function at the ice surface \\
\hline$b$ & $z$-coordinate of the ice base (lithosphere surface) \\
\hline$b_{0}$ & relaxed value for $b$ without ice load \\
\hline$b_{r}$ & $z$-coordinate of the lithosphere base \\
\hline$b_{\mathrm{ss}}$ & steady-state position of the lithosphere surface $b$ \\
\hline$C$ & sliding function for a cold ice base \\
\hline$C_{t}$ & sliding function for a temperate ice base \\
\hline$C_{\mathrm{sl}}$ & coefficient in the Weertman-type sliding law for a temperate ice base \\
\hline$c$ & specific heat of ice \\
\hline$c_{r}$ & specific heat of the lithosphere \\
\hline$D$ & strain-rate tensor \\
\hline$D(\omega)$ & water drainage function \\
\hline$E$ & enhancement factor in the flow law \\
\hline$f(\sigma), f_{t}(\sigma)$ & creep response function of cold and temperate ice \\
\hline$g$ & gravity acceleration \\
\hline$h$ & $z$-coordinate of the free ice surface \\
\hline$h_{\max }$ & maximum $h$ of the entire ice sheet \\
\hline$H$ & ice thickness \\
\hline$H_{\max }$ & maximum ice thickness $H$ \\
\hline$H_{\mathrm{t}, \max }$ & maximum thickness of the temperate ice layer \\
\hline$H_{r}$ & lithosphere thickness \\
\hline$j$ & diffusive water mass flux \\
\hline$j_{\text {tot }}$ & total water mass flux (relative to the motion of the CTS) \\
\hline$L$ & latent heat of ice \\
\hline M & water mass production rate in temperate ice \\
\hline$M_{s}$ & ablation rate (melting rate at the ice surface) \\
\hline$\dot{m}_{b}^{w}$ & water mass flux into the base \\
\hline$p$ & pressure \\
\hline$q$ & total heat flux, $\boldsymbol{q}_{s}+\boldsymbol{q}_{l}$ \\
\hline $\boldsymbol{q}_{l}$ & latent heat flux \\
\hline & sensible heat flux \\
\hline$Q, Q_{i}$ & horizontal mass flux, its $i$-th component \\
\hline$Q_{\text {geoth }}^{\perp}$ & geothermal heat flux \\
\hline
\end{tabular}




\begin{tabular}{|c|c|}
\hline$R$ & universal gas constant \\
\hline$S_{s}$ & accumulation rate (snowfall rate at the ice surface) \\
\hline$t$ & time \\
\hline$T$ & temperature \\
\hline$T_{M}$ & pressure melting temperature \\
\hline$T_{0}$ & melting temperature at zero pressure \\
\hline$T^{\prime}$ & homologous temperature $\left(T-T_{M}\right)$ \\
\hline$T_{\mathrm{ma}}$ & mean annual air temperature above the ice sheet \\
\hline$T_{s}$ & ice surface temperature (10-m firn temperature) \\
\hline$T$ & stress tensor \\
\hline $\boldsymbol{T}^{R}$ & stress deviator (frictional stresses) \\
\hline $\boldsymbol{v}, v_{i}$ & velocity (barycentric), its $i$-th component \\
\hline $\boldsymbol{v}_{b},\left(v_{b}\right)_{i}$ & basal ice velocity (barycentric), its $i$-th component \\
\hline $\boldsymbol{v}_{\mathrm{sl}},\left(v_{\mathrm{sl}}\right)_{i}$ & $\begin{array}{l}\text { basal sliding velocity (difference between ice velocity } \\
\text { and lithosphere velocity), its } i \text {-th component }\end{array}$ \\
\hline $\boldsymbol{v}_{i},\left(v_{i}\right)_{j}$ & ice velocity in the mixture ice plus water, its $j$-th component \\
\hline $\boldsymbol{v}_{w},\left(v_{w}\right)_{j}$ & water velocity in the mixture ice plus water, its $j$-th component \\
\hline$V_{\text {tot }}$ & total ice volume \\
\hline$V_{\text {temp }}$ & volume of temperate ice \\
\hline $\boldsymbol{w}, w_{i}$ & velocity of a singular surface, its $i$-th component \\
\hline$x, y$ & horizontal Cartesian coordinates \\
\hline$z$ & vertical Cartesian coordinate (elevation above sea level) \\
\hline$z_{m}$ & $z$ coordinate of the CTS \\
\hline$\alpha$ & ratio of potential energy to internal energy in cold ice \\
\hline$\alpha_{t}$ & ratio of potential energy to internal energy in temperate ice \\
\hline$\beta$ & Clausius-Clapeyron gradient \\
\hline$\epsilon$ & internal energy \\
\hline$\varepsilon$ & aspect ratio \\
\hline$\kappa$ & heat conductivity of ice \\
\hline$\kappa_{r}$ & heat conductivity of the lithosphere \\
\hline$\nu$ & water diffusivity in temperate ice \\
\hline$\rho$ & true density of ice and of the mixture ice plus water, respectively \\
\hline$\rho_{i}$ & partial density of ice in the mixture ice plus water \\
\hline$\rho_{w}$ & partial density of water in the mixture ice plus water \\
\hline$\rho_{a}$ & true density of the asthenosphere \\
\hline$\rho_{r}$ & true density of the lithosphere \\
\hline$\sigma$ & effective shear stress \\
\hline$\sigma_{i j}$ & $i j$-th component of the stress tensor \\
\hline$\sigma_{i j}^{R}$ & $i j$-th component of the stress deviator \\
\hline$\tau_{V}$ & time lag for isostatic bed adjustment \\
\hline$\omega$ & water content of temperate ice (mass fraction) \\
\hline$\omega_{\max }$ & threshold value in the water drainage law \\
\hline$[A]$ & typical rate factor \\
\hline$[c]$ & typical specific heat of ice \\
\hline$\left[c_{r}\right]$ & typical specific heat of the lithosphere \\
\hline$[C]$ & typical sliding function for a cold ice base \\
\hline$\left[C_{t}\right]$ & typical sliding function for a temperate ice base \\
\hline$[f]$ & typical creep response function \\
\hline$[H]$ & typical vertical dimension \\
\hline$[L]$ & typical horizontal dimension \\
\hline$\left[Q_{\text {geoth }}^{\perp}\right]$ & typical geothermal heat flux \\
\hline$\left[V_{H}\right]$ & typical vertical velocity \\
\hline$\left[V_{L}\right]$ & typical horizontal velocity \\
\hline$[\Delta T]$ & typical temperature difference \\
\hline
\end{tabular}




$\begin{array}{ll}{[\kappa]} & \text { typical heat conductivity of ice } \\ {\left[\kappa_{r}\right]} & \text { typical heat conductivity of the lithosphere } \\ {[\omega]} & \text { typical water content } \\ \mathcal{B} & \text { Clausius-Clapeyron number } \\ \mathcal{D} & \text { heat diffusion number } \\ \mathcal{D}_{t} & \text { water diffusion number } \\ \mathcal{D}_{r} & \text { heat diffusion number of the lithosphere } \\ \mathcal{F} & \text { sliding number for a cold ice base } \\ \mathcal{F}_{t} & \text { sliding number for a temperate ice base } \\ \mathcal{K} & \text { fluidity number } \\ \mathcal{N}_{r} & \text { geothermal heat number of the lithosphere } \\ \mathcal{P}_{b}^{w} & \text { basal melting rate } \\ \mathcal{P}_{m}^{w} & \text { water surface production rate at the CTS } \\ \mathcal{T}_{r} & \text { time-lag number for isostatic bed adjustment } \\ \mathrm{F} & \text { Froude number }\end{array}$

\section{B Integration of the slab equations}

The equations derived in $\S 3.1$, that result from applying the polythermal ice sheet model of $\S 2$ to the simplified problem for a sheet of uniform thickness, can be solved almost entirely analytically. Only for the CTS position $z=z_{m}$ does an implicit algebraic equation remain, which can easily be solved by a Newtonian root finder.

In view of (3.5), (3.6) and (3.7), integration of (3.2) yields

$$
\begin{gathered}
p(z)=\rho g \cos \gamma(H-z), \\
\sigma_{x z}(z)=\rho g \sin \gamma(H-z),
\end{gathered}
$$

and thus

$$
\sigma=\sigma_{x z}=\rho g \sin \gamma(H-z) .
$$

Obviously, the pressure is purely hydrostatic.

With this result, eqs. (3.1), (3.5), (3.6), (3.7) and a prescribed basal velocity $v_{x, b}$, the velocity distribution is

$$
\begin{gathered}
v_{x}(z)=\frac{A}{2}(\rho g \sin \gamma)^{3}\left[H^{4}-(H-z)^{4}\right]+v_{x, b}, \\
v_{z}(z)=\mathrm{const}=-a_{s}^{\perp}=-a_{m}^{\perp} .
\end{gathered}
$$

The velocity parallel to the bed, $v_{x}$, increases monotonically from its minimum value $v_{x, b}$ at the base to its maximum value at the free surface, as is expected for this gravity-driven shear flow. The velocity perpendicular to the bed, $v_{z}$, is constant; it balances the accumulation-ablation function $a_{s}^{\perp}$ at the free surface, and represents the negative ice volume flux $a_{m}^{\perp}$ through the CTS.

The construction of the solution of eqs. (3.3) and (3.4) for the temperature and the water content in the cold and the temperate region, respectively, and the associated determination of the CTS position, is a rather lenthy procedure. First, inserting eqs. (B.3) for $\sigma$ and (B.5) for $v_{z}$,

$$
\kappa \frac{d^{2} T}{d z^{2}}+\rho c a_{s}^{\perp} \frac{d T}{d z}=-2 A(\rho g \sin \gamma)^{4}(H-z)^{4}
$$

and

$$
\rho a_{s}^{\perp} \frac{d \omega}{d z}=-2 \frac{A}{L}(\rho g \sin \gamma)^{4}(H-z)^{4} .
$$

For easier calculation, map the vertical coordinate $z$ onto the interval $[0,1]$ by the transformation $z=H \zeta$, then

$$
D \frac{d^{2} T}{d \zeta^{2}}+M \frac{d T}{d \zeta}=-K(1-\zeta)^{4}
$$


and

$$
M \frac{d \omega}{d \zeta}=-K_{t}(1-\zeta)^{4}
$$

where

$$
\begin{aligned}
D & =\frac{\kappa}{\rho c}, \\
M & =H a_{s}^{\perp}, \\
K & =\frac{2 A}{\rho c} H^{6}(\rho g \sin \gamma)^{4}, \\
K_{t} & =\frac{2 A}{\rho L} H^{6}(\rho g \sin \gamma)^{4}
\end{aligned}
$$

Solution of the homogeneous equation of (B.8) is simply

$$
T_{h}=c_{1} e^{-(M / D) \zeta}+c_{2}
$$

A particular integral of the inhomogeneous equation has the form

$$
T_{p}=a_{1} \zeta+a_{2} \zeta^{2}+a_{3} \zeta^{3}+a_{4} \zeta^{4}+a_{5} \zeta^{5}
$$

where the coefficients $a_{1}$ to $a_{5}$ are calculated by balancing powers of $\zeta$ in (B.8), yielding

$$
\begin{aligned}
a_{5} & =-\frac{K}{5 M}, \\
a_{4} & =\frac{K}{M}+\frac{D K}{M^{2}}, \\
a_{3} & =-2 \frac{K}{M}-4 \frac{D K}{M^{2}}-4 \frac{D^{2} K}{M^{3}}, \\
a_{2} & =2 \frac{K}{M}+6 \frac{D K}{M^{2}}+12 \frac{D^{2} K}{M^{3}}+12 \frac{D^{3} K}{M^{4}}, \\
a_{1} & =-\frac{K}{M}-4 \frac{D K}{M^{2}}-12 \frac{D^{2} K}{M^{3}}-24 \frac{D^{3} K}{M^{4}}-24 \frac{D^{4} K}{M^{5}} .
\end{aligned}
$$

With the above results, the general solution of the temperature equation (B.8) is

$$
T=c_{1} e^{-(M / D) \zeta}+c_{2}+a_{1} \zeta+a_{2} \zeta^{2}+a_{3} \zeta^{3}+a_{4} \zeta^{4}+a_{5} \zeta^{5}
$$

The constants $c_{1}$ and $c_{2}$ are still free and must be determined by boundary and transition conditions.

First consider the water content equation (B.9), which can be integrated directly:

$$
\omega=\frac{K_{t}}{5 M}(1-\zeta)^{5}+c_{3}
$$

leaving a further undetermined. The last step consists of calculating the integration constants $c_{1}, c_{2}$ and $c_{3}$, and the CTS position $\zeta_{m}$. This must be performed separately for the cases of melting conditions $\left(a_{m}^{\perp}>0\right)$ and freezing conditions $\left(a_{m}^{\perp}<0\right)$ at the CTS.

\section{Slab with melting condition at the CTS:}

In this case, according to (B.5), the velocity perpendicular to the bed is negative, i.e., the ice flows from the free surface toward the base. Because of (3.6), (3.7) and the subsequent discussion,

$$
T(1)=T_{s}, \quad T^{+}\left(\zeta_{m}\right)=0, \quad\left(d T^{+} / d \zeta\right)_{\zeta_{m}}=0, \quad \omega^{-}\left(\zeta_{m}\right)=0 .
$$


Insertion of the first three of these equations into the general temperature solution (B.14) yields

$$
\begin{aligned}
T_{s} & =c_{1} e^{-(M / D)}+c_{2}+a_{1}+a_{2}+a_{3}+a_{4}+a_{5} \\
0 & =c_{1} e^{-(M / D) \zeta_{m}}+c_{2}+a_{1} \zeta_{m}+a_{2} \zeta_{m}^{2}+a_{3} \zeta_{m}^{3}+a_{4} \zeta_{m}^{4}+a_{5} \zeta_{m}^{5} \\
0 & =-\frac{M}{D} c_{1} e^{-(M / D) \zeta_{m}}+a_{1}+2 a_{2} \zeta_{m}+3 a_{3} \zeta_{m}^{2}+4 a_{4} \zeta_{m}^{3}+5 a_{5} \zeta_{m}^{4}
\end{aligned}
$$

which are three equations for the three unknowns $c_{1}, c_{2}$ and $\zeta_{m}$. With $c_{2}$ from (B.18) and $c_{1}$ from (B.19), (B.17) becomes an implicit algebraic equation for the CTS position $\zeta_{m}$,

$$
\begin{aligned}
0= & \frac{D}{M}\left(1-e^{(M / D)\left(\zeta_{m}-1\right)}\right)\left(a_{1}+2 a_{2} \zeta_{m}+3 a_{3} \zeta_{m}^{2}+4 a_{4} \zeta_{m}^{3}+5 a_{5} \zeta_{m}^{4}\right)+T_{s} \\
& +a_{1}\left(\zeta_{m}-1\right)+a_{2}\left(\zeta_{m}^{2}-1\right)+a_{3}\left(\zeta_{m}^{3}-1\right)+a_{4}\left(\zeta_{m}^{4}-1\right)+a_{5}\left(\zeta_{m}^{5}-1\right) \\
=: & f\left(\zeta_{m}\right) .
\end{aligned}
$$

This can be easily solved with a Newtonian root finder (starting with an estimated $\zeta_{m}^{(0)}$, then iterating by $\left.\zeta_{m}^{(n+1)}=\zeta_{m}^{(n)}-f\left(\zeta_{m}^{(n)}\right) / f^{\prime}\left(\zeta_{m}^{(n)}\right)\right)$, which yields $\zeta_{m}$ with great accuracy. This is the only step in the whole solution procedure that must be performed numerically. Now $c_{1}$ follows from eq. (B.19), and then $c_{2}$ from eq. (B.17). The temperature in the cold region, given by (B.14), is therefore determined completely.

The forth boundary condition of (B.16) determines the coefficient $c_{3}$ in the expression (B.15) for the water content, which becomes

$$
\omega=\frac{K_{t}}{5 M}\left[(1-\zeta)^{5}-\left(1-\zeta_{m}\right)^{5}\right]
$$

\section{Slab with freezing condition at the CTS:}

In this case, the velocity perpendicular to the bed is positive due to (B.5). The streamlines of the ice flow therefore run away from the base into the direction of the free surface. The boundary conditions, by eqs. (3.6), (3.7) and the subsequent discussion, are

$$
T(1)=T_{s}, \quad T^{+}\left(\zeta_{m}\right)=0, \quad \frac{\kappa}{H} \frac{d T^{+}}{d \zeta}=L \omega^{-} \rho a_{m}^{\perp}, \quad \omega(0)=0 .
$$

It is convenient here to first determine $c_{3}$ by the last condition, when (B.15) becomes

$$
\omega=\frac{K_{t}}{5 M}\left[(1-\zeta)^{5}-1\right]
$$

Thus the water content at the temperate side of the CTS is

$$
\omega^{-}=\omega\left(\zeta_{m}\right)=\frac{K_{t}}{5 M}\left[\left(1-\zeta_{m}\right)^{5}-1\right]
$$

where, however, $\zeta_{m}$ is still undetermined. Now, the temperature-gradient condition becomes

$$
\begin{aligned}
\frac{d T^{+}}{d \zeta} & =\frac{H}{\kappa} L \rho a_{m}^{\perp} \frac{K_{t}}{5 M}\left[\left(1-\zeta_{m}\right)^{5}-1\right] \\
& =\frac{L \rho K_{t}}{5 \kappa}\left[\left(1-\zeta_{m}\right)^{5}-1\right], \quad \text { since } \quad M=H a_{s}^{\perp}=H a_{m}^{\perp}
\end{aligned}
$$

The three temperature conditions (B.22) $)_{1,2}$ and (B.25) now relate $c_{1}, c_{2}$ and $\zeta_{m}$ :

$$
\begin{aligned}
T_{s}= & c_{1} e^{-(M / D)}+c_{2}+a_{1}+a_{2}+a_{3}+a_{4}+a_{5} \\
0= & c_{1} e^{-(M / D) \zeta_{m}}+c_{2} \\
& +a_{1} \zeta_{m}+a_{2} \zeta_{m}^{2}+a_{3} \zeta_{m}^{3}+a_{4} \zeta_{m}^{4}+a_{5} \zeta_{m}^{5} \\
\frac{L \rho K_{t}}{5 \kappa}\left[\left(1-\zeta_{m}\right)^{5}-1\right]= & -\frac{M}{D} c_{1} e^{-(M / D) \zeta_{m}}
\end{aligned}
$$




$$
+a_{1}+2 a_{2} \zeta_{m}+3 a_{3} \zeta_{m}^{2}+4 a_{4} \zeta_{m}^{3}+5 a_{5} \zeta_{m}^{4}
$$

Again, with $c_{2}$ from (B.27) and $c_{1}$ from (B.28), (B.26) becomes an implicit algebraic equation for $\zeta_{m}$,

$$
\begin{aligned}
0= & \frac{D}{M}\left(1-e^{(M / D)\left(\zeta_{m}-1\right)}\right)\left(a_{1}+2 a_{2} \zeta_{m}+3 a_{3} \zeta_{m}^{2}+4 a_{4} \zeta_{m}^{3}+5 a_{5} \zeta_{m}^{4}\right. \\
& \left.-\frac{L \rho K_{t}}{5 \kappa}\left[\left(1-\zeta_{m}\right)^{5}-1\right]\right)+T_{s} \\
& +a_{1}\left(\zeta_{m}-1\right)+a_{2}\left(\zeta_{m}^{2}-1\right)+a_{3}\left(\zeta_{m}^{3}-1\right)+a_{4}\left(\zeta_{m}^{4}-1\right)+a_{5}\left(\zeta_{m}^{5}-1\right) \\
=: & g\left(\zeta_{m}\right) .
\end{aligned}
$$

After solution by a Newtonian root finder, $c_{1}$ follows from (B.28), $c_{2}$ from (B.26), and the temperature distribution (B.14) is fully determined.

\section{References}

Abe-Ouchi, A. 1993. Ice sheet response to climatic changes: a modelling approach. Zürcher Geogr. Schr., 54, 134 pp.

Barenblatt, G. I. 1987. Dimensional analysis. Gordon and Breach Science Publ., New York etc.

Bauer, G. 1997. Thermomechanik von Mischungen. Pending Ph. D. thesis, Institut für Mechanik, Technische Hochschule Darmstadt, Germany.

Blatter, H. 1991. Effect of climate on the cryosphere. Zürcher Geogr. Schr., 41, 98 pp.

Budd, W. F. and I. N. Smith. 1982. Large-scale numerical modelling of the Antarctic ice sheet. Ann. Glaciol., 3, 42-49.

Calov, R. 1994. Das thermomechanische Verhalten des grönländischen Eisschildes unter der Wirkung verschiedener Klimaszenarien - Antworten eines theoretisch-numerischen Modells. Ph. D. thesis, Institut für Mechanik, Technische Hochschule Darmstadt, Germany, 171 pp.

Calov, R. and K. Hutter. 1996. The thermomechanical response of the Greenland ice sheet to various climate scenarios. Climate Dynamics, 12, 243-260.

Fabré, A., A. Letréguilly, C. Ritz and A. Mangeney. 1995. Greenland under changing climates: sensitivity experiments with a new three-dimensional ice sheet model. Ann. Glaciol., 21, 1-7.

Fastook, J. L. and J. E. Chapman. 1989. A map-plane finite-element model: three modeling experiments. J. Glaciol., 35 (119), 48-52.

Fowler, A. C. 1984. On the transport of moisture in polythermal glaciers. J. Geophys. Astrophys. Fluid Dyn., 28, 99-140.

Fowler, A. C. 1992. Modelling ice sheet dynamics. J. Geophys. Astrophys. Fluid Dyn., 63, 29-65.

Fowler, A. C. and D. A. Larson. 1978. On the flow of polythermal glaciers. I. Model and preliminary analysis. Proc. R. Soc. Lond. A, 363, 217-242.

Funk, M., K. Echelmeyer and A. Iken. 1994. Mechanisms of fast flow in Jacobshavns Isbræ, West Greenland: Part II. Modeling of englacial temperatures. J. Glaciol., 40 (136), 569-585.

Glen, J. W. 1955. The creep of polycrystalline ice. Proc. R. Soc. Lond. A, 228 (1175), 519-538.

Greve, R. 1995. Thermomechanisches Verhalten polythermer Eisschilde - Theorie, Analytik, Numerik. Ber. Geowissenschaft, Shaker Verlag, Aachen, Germany, 226 pp. (Ph. D. thesis, Institut für Mechanik, Technische Hochschule Darmstadt, Germany.) 
Greve, R. 1997. Application of a polythermal three-dimensional ice sheet model to the Greenland ice sheet: Response to steady-state and transient climate scenarios. J. Climate, 10 (5), 901-918.

Greve, R. and K. Hutter. 1995. Polythermal three-dimensional modelling of the Greenland ice sheet with varied geothermal heat flux. Ann. Glaciol., 21, 8-12.

Greve, R. and D. R. MacAyeal. 1996. Dynamic/thermodynamic simulations of Laurentide ice sheet instability. Ann. Glaciol., 23, 328-335 .

Greve, R., M. Weis and K. Hutter. 1997. Palaeoclimatic evolution and present conditions of the Greenland ice sheet in the vicinity of Summit: An approach by large-scale modelling. Palaeoclimates (submitted).

Hansen, I. and R. Greve. 1996. Polythermal modelling of steady states of the Antarctic Ice Sheet in comparison with the real world. Ann. Glaciol., 23, 382-387.

Hansen, I., R. Greve and K. Hutter. 1996. Application of a polythermal ice sheet model to the Antarctic Ice Sheet: Steady-state solution and response to Milanković cycles. Proceedings of the 5th International Symposium on Thermal Engineering and Science for Cold Regions, 89-96.

Herterich, K. 1988. A three-dimensional model of the Antarctic ice sheet. Ann. Glaciol., 11, 32-35.

Herterich, K. 1990. Modellierung eiszeitlicher Klimaschwankungen. Habilitation thesis, Fachbereich Geowissenschaften, Universität Hamburg, Germany, 209 pp.

Hooke, R. L. 1981. Flow law for polycrystalline ice in glaciers: comparison of theoretical predictions, laboratory data, and field measurements. Rev. Geophys. Space Phys., 19 (4), 664-672.

Hutter, K. 1982. A mathematical model of polythermal glaciers and ice sheets. J. Geophys. Astrophys. Fluid Dyn., 21, 201-224.

Hutter, K. 1983. Theoretical glaciology; material science of ice and the mechanics of glaciers and ice sheets. D. Reidel Publishing Company, Dordrecht, Holland, 510 pp.

Hutter, K. 1993. Thermo-mechanically coupled ice sheet response. Cold, polythermal, temperate. J. Glaciol., 39 (131), 65-86.

Hutter, K., H. Blatter and M. Funk. 1988. A model computation of moisture content in polythermal glaciers. J. Geophys. Res., 93 (B10), 12205-12214.

Hutter, K., S. Yakowitz and F. Szidarovszky. 1986. A numerical study of plane ice sheet flow. J. Glaciol., 32 (111), 139-164.

Huybrechts, P. 1992. The Antarctic ice sheet and environmental change: a three-dimensional modelling study. Ber. Polarforschung, 99, 241 pp.

Huybrechts, P. 1993. Glaciological modelling of the Late Cenozoic East Antarctic ice sheet: stability or dynamism? Geografiska Annaler, 75 A (4), 221-238.

Huybrechts, P. 1994. The present evolution of the Greenland ice sheet: an assessment by modelling. Global Planet. Change, 9, 39-51.

Huybrechts, P. and J. Oerlemans. 1988. Evolution of the East Antarctic ice sheet: A numerical study of thermo-mechanically response patterns with changing climate. Ann. Glaciol., 11, 52-59.

Jenssen, D. 1977. A three-dimensional polar ice-sheet model. J. Glaciol., 18 (80), 373-389.

Lee, W. H. K. 1970. On the global variations of terrestrial heat flow. Phys. Earth Planet. Inter., 2 (5), $332-341$.

Letréguilly, A., P. Huybrechts and N. Reeh. 1991a. Steady-state characteristics of the Greenland ice sheet under different climates. J. Glaciol. 37 (125), 149-157. 
Letréguilly, A., N. Reeh and P. Huybrechts. 1991b. The Greenland ice sheet through the last glacialinterglacial cycle. Palaeogeogr., Palaeoclimatol., Palaeoecol. (Global Planet. Change Sect.), 90, 385394.

Lliboutry, L. and P. Duval. 1985. Various isotropic and anisotropic ices found in glaciers and polar ice caps and their corresponding rheologies. Ann. Geophys., 3 (2), 207-224.

Mahaffy, M. W. 1976. A three-dimensional numerical model of ice sheets: Test on the Barnes ice cap, Northwest Territories. J. Geophys. Res., 81 (6), 1059-1066.

Morland, L. W. 1984. Thermo-mechanical balances of ice sheet flows. J. Geophys. Astrophys. Fluid Dyn., 29, 237-266.

Morland, L. W. 1993. The flow of ice sheets and ice shelves. Continuum mechanics in environmental sciences and geophysics, K. Hutter (ed.), CISM courses and lectures, 337, Springer Verlag, Wien etc., $403-466$.

Morland, L. W. and I. R. Johnsen. 1980. Steady motion of ice sheets. J. Glaciology, 25, 229-246.

Müller, I. 1985. Thermodynamics. Pitman Advanced Publishing Program, Boston etc., 521 pp.

Nye, J. F. 1957. The distribution of stress and velocity in glaciers and ice-sheets. Proc. R. Soc. Lond. A, 239, 113-133.

Oerlemans, J. 1982. Response of the Antarctic Ice Sheet to a climatic warming: a model study. J. Climatol., 2, 1-11.

Ohmura, A. 1987. New temperature distribution maps for Greenland. Z. Gletscherkd. Glazialgeol., 23, $1-45$.

Ohmura, A. and N. Reeh. 1991. New precipitation and accumulation maps for Greenland. J. Glaciol., 37, $140-148$.

Paterson, W. S. B. 1991. Why ice-age ice is sometimes "soft". Cold Reg. Sci. Technol., 20, 75-98.

Paterson, W. S. B. 1994. The physics of glaciers. Third edition. Oxford, etc., Pergamon Press, 480 pp.

Ritz, C. 1987. Time dependent boundary conditions for calculation of temperature fields in ice sheets. The physical basis of ice sheet modelling, E. D. Waddington and J. S. Walder (eds.), IAHS Publication 170, 207-216.

Svendsen, B. and K. Hutter. 1996. A continuum approach for modelling induced anisotropy in glaciers and ice sheets. Ann. Glaciol., 23, 262-269.

Svendsen, B. and K. Hutter. 1997. On the continuum modeling of induced anisotropy in polycrystals. Quart. J. Appl. Math. (submitted).

Wu, T. 1996. Schwerkraftgetriebene Scherströmungen in gesättigten Binärmischungen nicht-Newtonscher Fluide mit Anwendungen auf das Fließen von sedimentverschmutztem Eis. Ph. D. thesis, Institut für Mechanik, Technische Hochschule Darmstadt, Germany, 142 pp. 